UCB-PTH-04/03

LBNL-54534

hep-th/0402108

\title{
A Basic Class of Twisted Open WZW Strings
}

\author{
M.B.Halpern ${ }^{1 a}$ and C. Helfgott ${ }^{1 b}$ \\ 1 Department of Physics, University of California and \\ Theoretical Physics Group, Lawrence Berkeley National Laboratory \\ University of California, Berkeley, California 94720, USA
}

\begin{abstract}
Recently, Giusto and Halpern reported the open-string description of a certain basic class of untwisted open WZW strings, including their associated non-commutative geometry and open-string KZ equations. In this paper, we combine this development with results from the theory of current-algebraic orbifolds to find the open-string description of a corresponding basic class of twisted open WZW strings, which begin and end on different WZW branes. The basic class of twisted open WZW strings is in 1-to-1 correspondence with the twisted sectors of all closed-string WZW orbifolds, and moreover, the basic class can be decomposed into a large collection of open-string WZW orbifolds. At the classical level, these open-string orbifolds exhibit new twisted non-commutative geometries, and we also find the relevant twisted open-string $K Z$ equations which describe these orbifolds at the quantum level. In a related development, we also formulate the closed-string description (in terms of twisted boundary states) of the general twisted open WZW string.
\end{abstract}

${ }^{a}$ halpern@physics . berkeley .edu

${ }^{b}$ helfgott@socrates. berkeley.edu 


\section{Contents}

1 Introduetion 13

2 Preliminaries 4

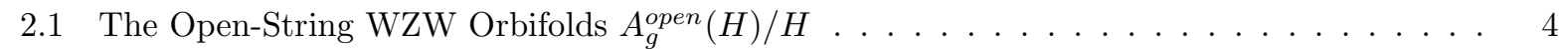

2.2 The Left Morer Data of the Closed Strine Orbifold $\Lambda(H) / H$. . . . . . . . . . . . . . . . . . 6

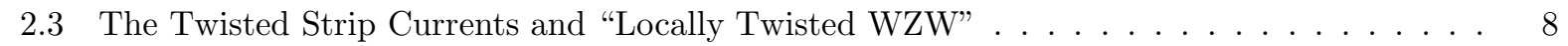

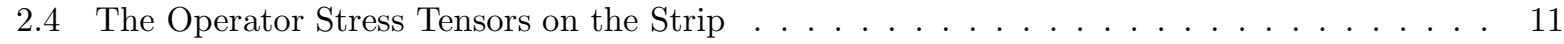

3 Classied Deseription of Twisted Open WZW Strings 13

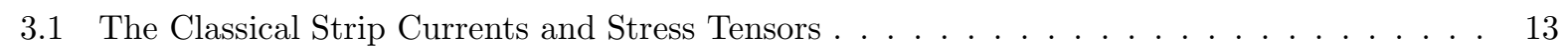

2.2 Phase Speepedization of the Strip Currents . . . . . . . . . . . . . . . . . . . 15

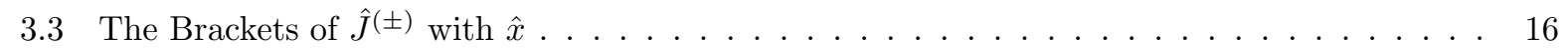

3.4 fordinte

3.5 The Generdized WZW Pranes 4 open $(H) / H$. . . . . . . . . . . . . . . . . . . . . 22

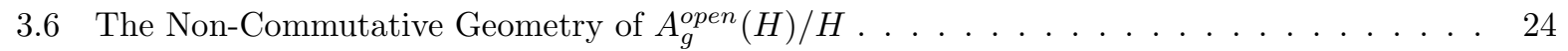

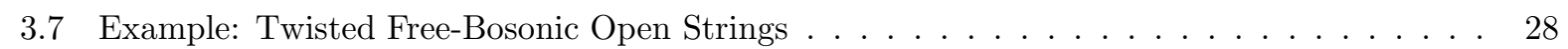

4 Conformal Field Theory of Twisted Open WZW Strimsis 32

4.1 The Quantum Hamilonian and the Twisted Afme Priman Fields . . . . . . . . . . . . . 33

4.2 Time Dependen of the Twioted Affe Primary Tield . . . . . . . . . . . . . . . . . . . . 34

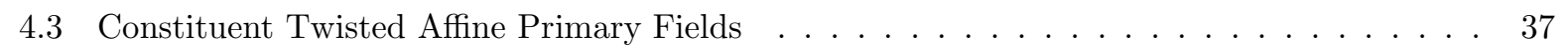

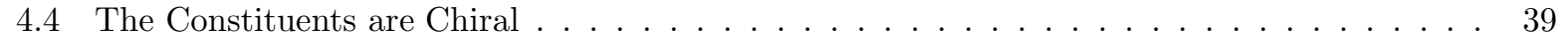

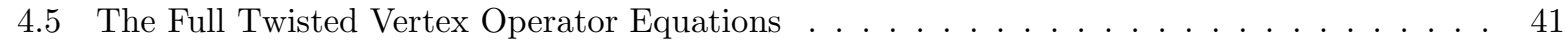

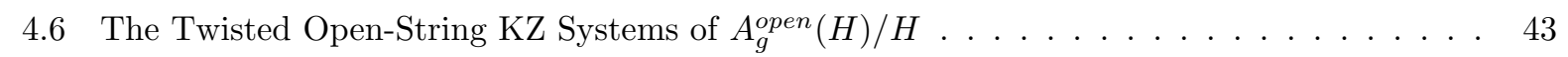

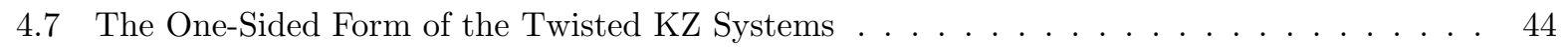

$\begin{array}{ll}5 . \text { Diseussion } & 47\end{array}$

A. General Twisted Boundary States 49

B Peetifieation in the Closed-Strins WZW Orbifolds 53

E More About the Phase-Modified Delta Funetions $\quad 57$

D Examples of the New Non-Gommutative Geometries 59

Referenees 


\section{Introduction}

In recent years, the orbifold program [1-11] has in large part completed the local description of closed-string orbifolds, including:

- the twisted current algebras and stress tensors of all sectors of the general currentalgebraic orbifold [1-5],

- the twisted affine primary fields, twisted operator algebras and twisted KZ equations [6, 7, 9, 10] of all WZW orbifolds,

- the action formulation $[6-8,10]$ of all WZW and coset orbifolds, in terms of group orbifold elements with definite monodromy,

- the action formulation and twisted Einstein equations [11] of a large class of sigma-model orbifolds,

- free-bosonic avatars [7, 9, 11] of these constructions and the explicit form of their twisted vertex operators.

A short review of the program can be found in Ref. 10. Recent progress at the level of characters has also been reported in Refs. [12,1,13-15].

Subsequently, the techniques of the orbifold program were also applied to construct a new class of so-called orientation orbifolds [16, 17, which arise by twisting world-sheet orientation-reversing automorphisms in closed-string WZW, coset and sigma models. Like conventional orientifolds [18-21], the orientation orbifolds contain both closed- and openstring sectors,but the orientation-orbifold sectors are characterized by fractional moding, including twisted Virasoro operators [1, 22, 9] in the open-string sectors.

The open-string sectors of the WZW orientation orbifolds are certainly not the most general twisted open WZW strings, but because orientation-reversing automorphisms are quite special, it is not immediately clear how to generalize the construction of Refs. [16, 17].

In pursuit of more general twisted open WZW strings, we have therefore reexamined the "open-string description" of untwisted open WZW strings ${ }^{\ddagger 1}$ given in Ref. [23], including the non-commutative geometry and the open-string $\mathrm{KZ}$ equations of these strings. We find that this construction can be straightforwardly combined with the theory of closed-string current-algebraic orbifolds to give a large set of new twisted open WZW strings, which we call the basic class.

\footnotetext{
${ }^{\ddagger 1}$ In the closed-string description of open strings, one uses the closed-string currents $J, \bar{J}$ to define boundary states, while in the open-string description of open strings, one works directly with a single set of current modes $J$.
} 
The basic class is a particular class of twisted open WZW strings, which

- is entirely disjoint from the open-string sectors of the WZW orientation orbifolds,

- is in 1-to-1 correspondence with the twisted sectors of all closed-string WZW orbifolds,

- can be decomposed into a large collection of open-string WZW orbifolds

$$
\frac{A_{g}^{\text {open }}(H)}{H}, \quad H \subset A u t(g)
$$

where $A_{g}^{\text {open }}(H)$ is any Giusto-Halpern open string with a symmetry $H$.

Examples of simple open-string orbifolds have been discussed e. g. in Refs. [24, 25].

An overview of our construction is given in Subsec. 2.1, and the necessary background material from the orbifold program is reviewed in Subsec. 2.2 and Apps. A,B. Central results for the basic class include the generalized WZW branes in Subsec. 3.5, the new twisted non-commutative geometry in Subsec. 3.6 and the twisted open-string KZ equations in Subsec. 4.5. Explicit non-abelian examples are given in Subsecs. 3.5, 4.5 and App. D, and free-bosonic analogues are worked out in Subsec. 3.7. In a parallel development, App. A formulates the closed-string description (in terms of twisted boundary states) of the general twisted open WZW string.

Taken together, the general twisted boundary state equation and the open-string sectors of the WZW orientation orbifolds give important clues for further generalization of our main development (see the final Discussion in Sec. 5). Based on these observations, we will return elsewhere to construct the open-string description of the general twisted open WZW string - which includes both the orientation orbifolds and the basic class as special cases.

\section{Preliminaries}

\subsection{The Open-String WZW Orbifolds $A_{g}^{\text {open }}(H) / H$}

In Ref. 23] a procedure was given to construct an open WZW string $A_{g}^{\text {open }}$ from the leftmover sector of the closed-string WZW model $A_{g}$ on affine $g$. In this paper we combine this procedure with the local theory of current-algebraic orbifolds [1-11] to construct a corresponding set of $N_{c}$ twisted open WZW strings, one from each of the left-mover sectors $\sigma=0 \ldots N_{c}-1$ of the general closed-string WZW orbifold $A_{g}(H) / H$ (see Fig. 11). 


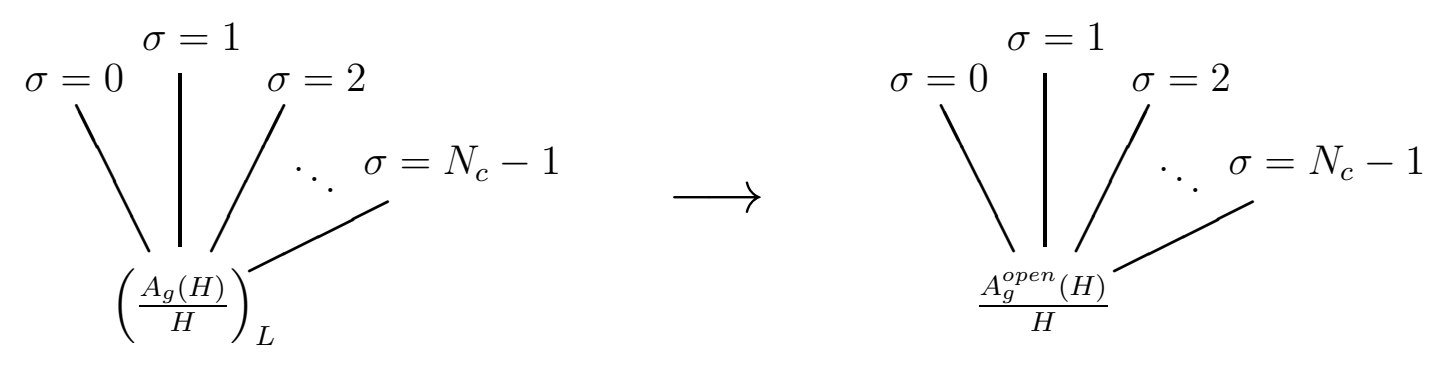

$\left(\frac{A_{g}(H)}{H}\right)_{L}:$ The left-mover data of any closed-string WZW orbifold.

$\frac{A_{g}^{\text {open }}(H)}{H}$ : The corresponding open-string WZW orbifold.

$N_{c}$ : The number of conjugacy classes of symmetry group $H \subset A u t(g)$ and the number of sectors $\sigma$ of $A_{g}(H) / H$ or $A_{g}^{\text {open }}(H) / H$.

Fig.11. Construction of twisted open WZW strings from closed-string WZW orbifold

Although our construction follows the path shown in Fig. [1, it is also possible to consider these new constructions as open-string orbifolds $A_{g}^{\text {open }}(H) / H$ of any $H$-symmetric untwisted open WZW string $A_{g}^{\text {open }}(H)$ (see Fig. 2).

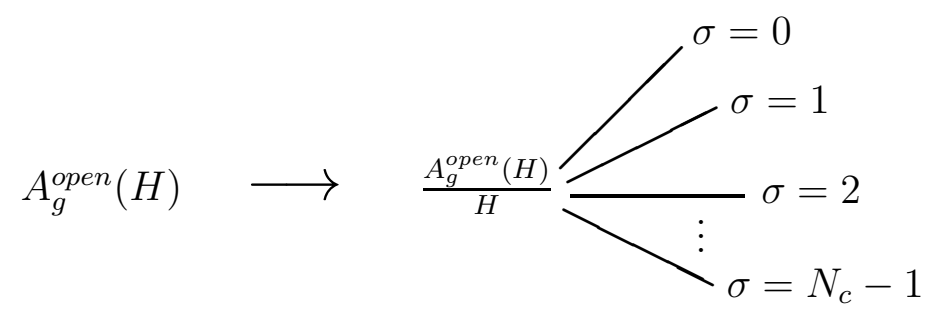

Fig.2. The open-string WZW orbifold $A_{g}^{\text {open }}(H) / H$ associated to the untwisted open WZW string $A_{g}^{\text {open }}(H)$ with a symmetry $H$.

We infer that $A_{g}^{\text {open }}(H) / H$ is an open-string WZW orbifold for the following reasons:

- Each sector $\sigma=0, \ldots, N_{c}-1$ of $A_{g}^{\text {open }}(H) / H$ is a twisted open string, in particular, the $\sigma=0$ sector is the untwisted open string $A_{g}^{\text {open }}(H)$ of Ref. 23] with a symmetry H.

- As in closed-string orbifold theory, each sector $\sigma$ of $A_{g}^{\text {open }}(H) / H$ is labelled by a conjugacy class of $H$. Moreover, sector $\sigma$ contains the appropriate twisted current algebra, obtained by twisting affine $g$ by an element $h_{\sigma} \in H$ in this conjugacy class.

- At the classical level, the target space of each sector of $A_{g}^{\text {open }}(H) / H$ is the appropriate sector of the group orbifold $g / H$, with the corresponding group orbifold elements [6-8], and with generalized WZW branes at each end of the open string. 
Because of modifications needed to apply the principle of local isomorphisms [3, 5, 6] to equal-time formulations [11] and open strings [16], a direct realization of the path shown in Fig. 2 will not be discussed in this paper.

In what follows we will therefore refer to $A_{g}^{\text {open }}(H) / H$ as an open-string WZW orbifold, while the basic class of twisted open strings will denote the set of all sectors of all $A_{g}^{\text {open }}(H) / H$. Presumably, these open-string WZW orbifolds are associated via non-planar processes to closed-string WZW orbifolds, but we will not study this issue here.

\subsection{The Left-Mover Data of the Closed-String Orbifold $A_{g}(H) / H$}

Ref. 23] constructed a basic class of untwisted open WZW strings from the untwisted affineSugawara construction [26-30] on affine $g$ [31, 32, 26], and it is clear from this development that an open-string conformal field theory can be constructed from any single chiral currentalgebraic stress tensor [26,27,33-38]

For the twisted construction of this paper, we therefore begin with the left-mover ${ }^{\ddagger 2}$ twisted affine-Sugawara construction [3, 5, 6] of sector $\sigma$ of the closed-string WZW orbifold $A_{g}(H) / H$ :

$$
\begin{gathered}
L_{\sigma}(m)=\mathcal{L}_{\hat{\mathfrak{g}}(\sigma)}^{n(r) \mu ;-n(r), \nu}(\sigma) \sum_{p \in \mathbb{Z}}: \hat{J}_{n(r) \mu}\left(p+\frac{n(r)}{\rho(\sigma)}\right) \hat{J}_{-n(r), \nu}\left(m-p-\frac{n(r)}{\rho(\sigma)}\right): \\
{\left[\hat{J}_{n(r) \mu}\left(m+\frac{n(r)}{\rho(\sigma)}\right), \hat{J}_{n(s) \nu}\left(n+\frac{n(s)}{\rho(\sigma)}\right)\right]=i \mathcal{F}_{n(r) \mu ; n(s) \nu}{ }^{n(r)+n(s), \delta}(\sigma) \hat{J}_{n(r)+n(s), \delta}\left(m+n+\frac{n(r)+n(s)}{\rho(\sigma)}\right)} \\
+\left(m+\frac{n(r)}{\rho(\sigma)}\right) \delta_{m+n+\frac{n(r)+n(s)}{\rho(\sigma)}, 0} \mathcal{G}_{n(r) \mu ;-n(r), \nu}(\sigma) \\
{\left[L_{\sigma}(m), \hat{J}_{n(r) \mu}\left(n+\frac{n(r)}{\rho(\sigma)}\right)\right]=-\left(n+\frac{n(r)}{\rho(\sigma)}\right) \hat{J}_{n(r) \mu}\left(m+n+\frac{n(r)}{\rho(\sigma)}\right)} \\
{\left[L_{\sigma}(m), L_{\sigma}(n)\right]=(m-n) L_{\sigma}(m+n)+\delta_{m+n, 0} \frac{\hat{c}}{12} m\left(m^{2}-1\right)} \\
\hat{c}=2 \mathcal{L}_{\hat{\mathfrak{g}}(\sigma)}^{n(r) \mu ;-n(r), \nu}(\sigma) \mathcal{G}_{n(r) \mu ;-n(r), \nu}(\sigma)=2 L_{g}^{a b} G_{a b}=c_{g}, \quad \sigma=0 \ldots N_{c}-1
\end{gathered}
$$

The normal ordering : · : in Eq. (2.1a) is the mode form of operator-product normal ordering in the orbifold [2, 3, 5, 6], and the alternate mode normal-ordered form : · $:_{M}$ of the twisted affine-Sugawara construction is given in Eq. (A.3).

\footnotetext{
${ }^{\ddagger 2}$ If the algebra of the twisted right-mover currents $\hat{\bar{J}}$ of $A_{g}(H) / H$ is not rectifiable [6, 7, 9, 10, into a copy of the twisted left-mover current algebra, then in principle there could be another set of twisted open strings based on $\hat{\bar{J}}$. However, as reviewed in App. B, all basic orbifold types are known to be rectifiable.
} 
The numerical coefficients in Eq. (2.1) are called twisted tensors or duality transformations. For sector $\sigma$ of $A_{g}(H) / H$, the standard duality transformations have the following explicit forms:

$$
\begin{aligned}
& \mathcal{G}_{n(r) \mu ; n(s) \nu}(\sigma) \equiv \chi(\sigma)_{n(r) \mu} \chi(\sigma)_{n(s) \nu} U(\sigma)_{n(r) \mu}{ }^{a} U(\sigma)_{n(s) \nu}{ }^{b} G_{a b} \\
& =\mathcal{G}_{n(s) \nu ; n(r) \mu}(\sigma)=\delta_{n(r)+n(s), 0 \bmod \rho(\sigma)} \mathcal{G}_{n(r) \mu ;-n(r), \nu}(\sigma) \\
& \mathcal{F}_{n(r) \mu ; n(s) \nu}{ }^{n(t) \delta}(\sigma) \equiv \chi(\sigma)_{n(r) \mu} \chi(\sigma)_{n(s) \nu} \chi(\sigma)_{n(t) \delta}^{-1} U(\sigma)_{n(r) \mu}{ }^{a} U(\sigma)_{n(s) \nu}{ }^{b} f_{a b}{ }^{c} U^{\dagger}(\sigma)_{c}{ }^{n(t) \delta} \\
& =-\mathcal{F}_{n(s) \nu ; n(r) \mu}{ }^{n(t) \delta}(\sigma)=\delta_{n(r)+n(s)-n(t), 0 \bmod \rho(\sigma)} \mathcal{F}_{n(r) \mu ; n(s) \nu}{ }^{n(r)+n(s), \delta}(\sigma) \\
& \mathcal{L}_{\hat{\mathfrak{g}}(\sigma)}^{n(r) \mu ; n(s) \nu}(\sigma)=\chi(\sigma)_{n(r) \mu}^{-1} \chi(\sigma)_{n(s) \nu}^{-1} L_{g}^{a b} U^{\dagger}(\sigma)_{a}{ }^{n(r) \mu} U^{\dagger}(\sigma)_{b}{ }^{n(s) \nu} \\
& =\mathcal{L}_{\hat{\mathfrak{g}}(\sigma)}^{n(s) \nu ; n(r) \mu}(\sigma)=\delta_{n(r)+n(s), 0 \bmod \rho(\sigma)} \mathcal{L}_{\hat{\mathfrak{g}}(\sigma)}^{n(r) \mu ;-n(r), \nu}(\sigma) \\
& \mathcal{T}_{n(r) \mu}(T, \sigma) \equiv \chi(\sigma)_{n(r) \mu} U(\sigma)_{n(r) \mu}{ }^{a} U(T, \sigma) T_{a} U^{\dagger}(T, \sigma) \\
& e^{2 \pi i \frac{n(r)}{\rho(\sigma)}} \mathcal{T}_{n(r) \mu}(T, \sigma)=E(T, \sigma) \mathcal{T}_{n(r) \mu}(T, \sigma) E(T, \sigma)^{*} . \\
& {\left[\mathcal{T}_{n(r) \mu}(T, \sigma), \mathcal{T}_{n(s) \nu}(T, \sigma)\right]=i \mathcal{F}_{n(r) \mu ; n(s) \nu}{ }^{n(r)+n(s), \delta}(\sigma) \mathcal{T}_{n(r)+n(s), \delta}(T, \sigma)} \\
& \widehat{\operatorname{Tr}}\left(\mathcal{M}(\mathcal{T}, \sigma) \mathcal{T}_{n(r) \mu}(T, \sigma) \mathcal{T}_{n(s) \nu}(T, \sigma)\right)=\mathcal{G}_{n(r) \mu ; n(s) \nu}(\sigma) \\
& g=\oplus_{I} \mathfrak{g}^{I}, \quad G_{a b}=\oplus_{I} k_{I} \eta_{a b}^{I}, \quad f_{a b}^{c}=\oplus_{I} f_{a b}^{I c}, \quad L_{g}^{a b}=\oplus_{I} \frac{\eta_{I}^{a b}}{2 k_{I}+Q_{I}}, \quad T_{a}=\oplus_{I} T_{a}^{I} .
\end{aligned}
$$

Here the quantities $G, f, L_{g}$ and $T$ are the generalized Killing metric, structure constants, inverse inertia tensor and representation matrices of the untwisted theory on $g$. The twisted tensors $\mathcal{G}, \mathcal{F}, \mathcal{L}$ and $\mathcal{T}$ are the duality transformations (discrete Fourier transforms) of the corresponding untwisted quantities. Similarly, the twisted data matrix $\mathcal{M}[6]$ is the duality transformation of the data matrix, which records the level $k_{I}$ of each affine $\mathfrak{g}^{I}$ and the Dynkin index of each rep $T^{I}$.

In Eq. (2.2), the normalization constants $\chi(\sigma)$ are essentially arbitrary and the unitary matrices (Fourier elements) $U(\sigma)$ and $U(T, \sigma)$ solve the $H$-eigenvalue problems [3, 5, 6, 7, 10] of orbifold theory:

$$
\begin{gathered}
\omega\left(h_{\sigma}\right)_{a}{ }^{b} U^{\dagger}(\sigma)_{b}{ }^{n(r) \mu}=U^{\dagger}(\sigma)_{a}{ }^{n(r) \mu} E_{n(r)}(\sigma), \quad E_{n(r)}(\sigma)=e^{-2 \pi i \frac{n(r)}{\rho(\sigma)}} \\
W\left(h_{\sigma} ; T\right)_{\alpha}{ }^{\beta} U^{\dagger}(T, \sigma)_{\beta}{ }^{N(r) \mu}=U^{\dagger}(T, \sigma)_{\alpha}{ }^{N(r) \mu} E_{N(r)}(T, \sigma), \quad E_{N(r)}(T, \sigma)=e^{-2 \pi i \frac{N(r)}{R(\sigma)}} \\
E(T, \sigma)_{N(r) \mu}{ }^{N(s) \nu}=\delta_{\mu}^{\nu} \delta_{N(r)+N(s), 0 \bmod R(\sigma)} E_{N(r)}(T, \sigma) \\
\sigma=0 \ldots N_{c}-1 .
\end{gathered}
$$


Here the matrices $\omega\left(h_{\sigma}\right)$ and $W\left(h_{\sigma} ; T\right)$ are the actions (in the underlying untwisted theory) of the automorphism $h_{\sigma} \in H$ in the adjoint rep and rep $T$ respectively, and $E_{n(r)}(\sigma)$, $E_{N(r)}(T, \sigma)$ are the eigenvalues of $\omega\left(h_{\sigma}\right), W\left(h_{\sigma} ; T\right)$. All these quantities are periodic $n(r) \rightarrow$ $n(r) \pm \rho(\sigma), N(r) \rightarrow N(r) \pm R(\sigma)$ in any spectral index, with period equal to the order $\rho(\sigma)$ or $R(\sigma)$ of the corresponding automorphic action $\omega$ or $W$. We denote the pullbacks to the fundamental ranges by the usual symbols $\bar{n}(r), \bar{N}(r)$.

In the untwisted sector $\sigma=0$, we have

$$
\begin{gathered}
\omega\left(h_{\sigma}\right)=W\left(h_{\sigma} ; T\right)=\mathbb{1}, \quad U(\sigma)=U(T, \sigma)=\mathbb{1}, \quad \chi(\sigma)=1 \\
\mathcal{G} \rightarrow G, \quad \mathcal{F} \rightarrow f, \quad \mathcal{L} \rightarrow L_{g}, \quad \mathcal{T} \rightarrow T
\end{gathered}
$$

and the system (2.1) and (2.2) reduces to the left-mover sector of the WZW model $A_{g}(H)$ with symmetry $H \subset \operatorname{Aut}(g)$. Except for the $H$-symmetry of the untwisted theory (which was modded out to construct the twisted sectors), this is precisely the starting point of Ref. [23].

The reader should therefore bear in mind that the $\sigma=0$ sectors of our open-string WZW orbifolds below will agree with all the untwisted results of Ref. [23] - although one should $H$-symmetrize the correlators of the untwisted string when it is included as an orbifold sector in our construction.

For detailed information on particular classes of closed-string WZW orbifolds, we direct the reader to the following references:

- the WZW permutation orbifolds [6, 7, , 9]

- the inner-automorphic WZW orbifolds [6, 9

- the (outer-automorphic) charge conjugation orbifold on $\mathfrak{s u}(n \geq 3)[7$

- the outer-automorphic WZW orbifolds on $\mathfrak{s o}(2 n)$, including the triality orbifolds on $\mathfrak{s o}(8)$ [10].

Ref. [10] also contains a short review of the program.

\subsection{The Twisted Strip Currents and "Locally Twisted WZW"}

Given the left-mover data of the previous subsection, we begin our construction of the open-string orbifold $A_{g}^{\text {open }}(H) / H$ by defining the twisted left- and right-mover open-string 
currents on the strip

$$
\begin{gathered}
\hat{J}_{n(r) \mu}^{( \pm)}(\xi, t, \sigma) \equiv \sum_{m \in \mathbb{Z}} \hat{J}_{n(r) \mu}\left(m+\frac{n(r)}{\rho(\sigma)}\right) e^{-i\left(m+\frac{n(r)}{\rho(\sigma)}\right)(t \pm \xi)}, \quad 0 \leq \xi \leq \pi \\
\hat{J}_{n(r) \mu}^{( \pm)}(-\xi, t, \sigma)=\hat{J}^{(\mp)}(\xi, t, \sigma) \\
\partial_{\mp} \hat{J}^{( \pm)}(\xi, t, \sigma)=0, \quad \partial_{ \pm}=\partial_{t} \pm \partial_{\xi}, \quad \sigma=0, \ldots, N_{c}-1
\end{gathered}
$$

both of which are constructed from the same set (2.1b) of twisted left-mover current modes. The strip currents satisfy the following boundary conditions

$$
\hat{J}_{n(r) \mu}^{(+)}(0, t, \sigma)=\hat{J}_{n(r) \mu}^{(-)}(0, t, \sigma), \quad \hat{J}_{n(r) \mu}^{(+)}(\pi, t, \sigma)=e^{-2 \pi i \frac{n(r)}{\rho(\sigma)}} \hat{J}_{n(r) \mu}^{(-)}(\pi, t, \sigma)
$$

which are the image on the strip of the monodromy (B.3c) of the corresponding cylinder current. With the mode expansion (2.5a) and the mode algebra (2.1b), we may compute the equal-time algebra of the strip currents

$$
\begin{aligned}
& {\left[\hat{J}_{n(r) \mu}^{(+)}(\xi, t, \sigma), \hat{J}_{n(s) \nu}^{(+)}(\eta, t, \sigma)\right]=2 \pi i\left(\mathcal{F}_{n(r) \mu ; n(s) \nu}{ }^{n(r)+n(s), \delta}(\sigma) \hat{J}_{n(r)+n(s), \delta}^{(+)}(\eta, t, \sigma)\right.} \\
& \left.+\delta_{n(r)+n(s), 0 \bmod \rho(\sigma)} \mathcal{G}_{n(r) \mu ;-n(r) \nu}(\sigma) \partial_{\xi}\right) \delta_{\frac{n(r)}{\rho(\sigma)}}(\xi-\eta) \\
& {\left[\hat{J}_{n(r) \mu}^{(+)}(\xi, t, \sigma), \hat{J}_{n(s) \nu}^{(-)}(\eta, t, \sigma)\right]=2 \pi i\left(\mathcal{F}_{n(r) \mu ; n(s) \nu}{ }^{n(r)+n(s), \delta}(\sigma) \hat{J}_{n(r)+n(s), \delta}^{(-)}(\eta, t, \sigma)\right.} \\
& \left.+\delta_{n(r)+n(s), 0 \bmod \rho(\sigma)} \mathcal{G}_{n(r) \mu ;-n(r) \nu}(\sigma) \partial_{\xi}\right) \delta_{\frac{n(r)}{\rho(\sigma)}}(\xi+\eta) \\
& {\left[\hat{J}_{n(r) \mu}^{(-)}(\xi, t, \sigma), \hat{J}_{n(s) \nu}^{(-)}(\eta, t, \sigma)\right]=2 \pi i\left(\mathcal{F}_{n(r) \mu ; n(s) \nu}{ }^{n(r)+n(s), \delta}(\sigma) \hat{J}_{n(r)+n(s), \delta}^{(-)}(\eta, t, \sigma)\right.} \\
& \left.-\delta_{n(r)+n(s), 0 \bmod \rho(\sigma)} \mathcal{G}_{n(r) \mu ;-n(r) \nu}(\sigma) \partial_{\xi}\right) \delta_{-\frac{n(r)}{\rho(\sigma)}}(\xi-\eta)
\end{aligned}
$$

where $\mathcal{G}(\sigma)$ and $\mathcal{F}(\sigma)$ are the twisted tangent-space metric and twisted structure constants given explicitly in Eq. (2.2).

The phase-modified Dirac delta functions $\delta_{n(r) / \rho(\sigma)}(\xi \pm \eta)$ in Eq. (2.7) are defined as 
follows:

$$
\begin{aligned}
& \delta_{\frac{n(r)}{\rho(\sigma)}}(\xi \pm \eta) \equiv e^{-i \frac{n(r)}{\rho(\sigma)}(\xi \pm \eta)} \delta(\xi \pm \eta)=\frac{1}{2 \pi} \sum_{m \in \mathbb{Z}} e^{-i\left(m+\frac{n(r)}{\rho(\sigma)}\right)(\xi \pm \eta)}=\delta_{-\frac{n(r)}{\rho(\sigma)}}(-\xi \mp \eta) \\
& \delta_{\frac{n(r) \pm \rho(\sigma)}{\rho(\sigma)}}(\xi \pm \eta)=\delta_{\frac{n(r)}{\rho(\sigma)}}(\xi \pm \eta), \quad \delta_{0}(\xi \pm \eta)=\delta(\xi \pm \eta) \\
& \delta_{\frac{n(r)}{\rho(\sigma)}}(\xi \pm \eta+2 \pi)=e^{-2 \pi i \frac{n(r)}{\rho(\sigma)}} \delta_{\frac{n(r)}{\rho(\sigma)}}(\xi \pm \eta) \\
& \delta(\xi \pm \eta) \equiv \frac{1}{2 \pi} \sum_{m \in \mathbb{Z}} e^{-i m(\xi \pm \eta)}, \quad \delta(\xi \pm \eta+2 \pi)=\delta(\xi \pm \eta)
\end{aligned}
$$

The quantity $\delta_{n(r) / \rho(\sigma)}(\xi-\eta)$ appeared previously in the orbifold geometry of Ref. [11], but the quantity $\delta_{n(r) / \rho(\sigma)}(\xi+\eta)$ appears for the first time here. We note in particular that $\delta_{n(r) / \rho(\sigma)}(\xi+\eta)$ has support only at the boundaries of the strip $\xi=\eta=0$ or $\pi$. The reader is referred to App. C for various useful identities involving these phase-modified delta functions.

Following Ref. 23], we compare the twisted open-string equal-time current algebra (2.7) on the strip to the twisted closed-string left- and right-mover current algebra (B.4) on the cylinder under the map:

$$
\text { (strip) } \hat{J}_{n(r) \mu}^{(+)}(\xi, t), \hat{J}_{n(r) \mu}^{(-)}(\xi, t) \stackrel{?}{\longleftrightarrow} \hat{J}_{n(r) \mu}(\xi, t), \hat{\bar{J}}_{n(r) \mu}(\xi, t) \text { (cylinder) } .
$$

We find that the two systems disagree by boundary terms in the left/right-mover commutators (B.4c) vs. (2.7b). More importantly, this comparison fails due to the form of the bulk terms $\left(\delta_{n(r) / \rho(\sigma)}(\xi-\eta)\right.$ vs. $\left.\delta_{-n(r) / \rho(\sigma)}(\xi-\eta)\right)$ in the right/right-mover commutators (B.4b) vs. (2.7c). We find however a successful comparison when we consider instead the rectified right-mover currents $\hat{\bar{J}}^{\sharp}$ reviewed in App. B:

$$
\text { (strip) } \hat{J}_{n(r) \mu}^{(+)}(\xi, t), \hat{J}_{n(r) \mu}^{(-)}(\xi, t) \longleftrightarrow \hat{J}_{n(r) \mu}(\xi, t), \hat{\bar{J}}_{n(r) \mu}^{\sharp}(\xi, t) \text { (cylinder) }
$$

In this case, the strip current algebra (2.7) is in fact isomorphic in the bulk to the rectified cylinder current algebra (B.6), the two algebras differing only by terms $\delta_{n(r) / \rho(\sigma)}(\xi+\eta)$ with support at the boundary.

In Ref. [23], the forms of various untwisted strip algebras were fixed by the requirement that these algebras must be locally isomorphic in the bulk to the untwisted algebras of the corresponding closed WZW string. The reason for this "locally WZW" requirement was that it guaranteed that all local quantities and relations (such as the action density and equations of motion) were isomorphic in the bulk to the corresponding relations in the closed WZW string, as expected intuitively [39]. 
Following this intuition, Eq. (2.10) tells us that the "locally WZW" requirement of Ref. 23] must be generalized to a new "locally twisted WZW" requirement for twisted open strings: All twisted strip algebras must be isomorphic in the bulk to the rectified twisted algebras of the corresponding closed-string WZW orbifold sector. Drawing on the rectified closed-string results collected in App. B, and using this requirement to fix certain results below, we will find that all local quantities and relations in the open-string WZW orbifold are indeed isomorphic in the bulk to the corresponding relations in the closed-string WZW orbifold. We note in particular that the locally twisted WZW condition reduces in untwisted sector $\sigma=0$ to the original locally WZW condition of Ref. [23].

\subsection{The Operator Stress Tensors on the Strip}

We turn now to the left- and right-mover stress tensors of sector $\sigma$ of the open-string orbifold $A_{g}^{\text {open }}(H) / H$

$$
\begin{gathered}
\hat{T}_{\sigma}^{( \pm)}(\xi, t) \equiv \frac{1}{2 \pi} \mathcal{L}_{\hat{\mathfrak{g}}(\sigma)}^{n(r) \mu ;-n(r), \nu}(\sigma): \hat{J}_{n(r) \mu}^{( \pm)}(\xi, t, \sigma) \hat{J}_{-n(r), \nu}^{( \pm)}(\xi, t, \sigma):, \quad 0 \leq \xi \leq \pi \\
=\frac{1}{2 \pi} \sum_{m \in \mathbb{Z}} L_{\sigma}(m) e^{-i m(t \pm \xi)} \\
\hat{T}_{\sigma}^{( \pm)}(-\xi, t)=\hat{T}_{\sigma}^{(\mp)}(\xi, t), \quad \partial_{\mp} \hat{T}_{\sigma}^{( \pm)}(\xi, t)=0, \quad \sigma=0, \ldots, N_{c}-1
\end{gathered}
$$

which are also constructed from the single left-mover stress tensor $\hat{T}_{\sigma}(\xi), 0 \leq \xi \leq 2 \pi$ of closed-string orbifold sector $\sigma$. The normal ordering : · : is the same as that shown in Eq. (2.1). With Eq. (B.10), we note that the forms of the strip stress tensors $\hat{T}^{( \pm)}$are locally twisted WZW

$$
\hat{J}^{(+)}, \hat{J}^{(-)}, \hat{T}_{\sigma}^{(+)}, \hat{T}_{\sigma}^{(-)} \longleftrightarrow \hat{J}, \hat{\bar{J}}^{\sharp}, \hat{T}_{\sigma}, \hat{\bar{T}}_{\sigma}
$$

that is, isomorphic in form to the left- and right-mover stress tensors $\hat{T}, \hat{\bar{T}}$ of the corresponding closed-string WZW orbifold sector.

The open-string stress tensors satisfy the following boundary conditions

$$
\hat{T}_{\sigma}^{(+)}(\xi, t)=\hat{T}_{\sigma}^{(-)}(\xi, t), \text { at } \xi=0, \pi
$$

which are obtained directly from Eq. (2.11b), or equivalently from (2.11a $)$ and the current boundary conditions (2.6).

The equal-time algebra of the open-string stress tensors

$$
\begin{aligned}
& {\left[\hat{T}_{\sigma}^{(+)}(\xi, t), \hat{T}_{\sigma}^{( \pm)}(\eta, t)\right]=i\left(\left(\hat{T}_{\sigma}^{(+)}(\xi, t)+\hat{T}_{\sigma}^{( \pm)}(\eta, t)\right)-\frac{c_{g}}{24 \pi}\left(\partial_{\xi}^{2}+1\right)\right) \partial_{\xi} \delta(\xi \mp \eta)} \\
& {\left[\hat{T}_{\sigma}^{(-)}(\xi, t), \hat{T}_{\sigma}^{( \pm)}(\eta, t)\right]=-i\left(\left(\hat{T}_{\sigma}^{(-)}(\xi, t)+\hat{T}_{\sigma}^{( \pm)}(\eta, t)\right)-\frac{c_{g}}{24 \pi}\left(\partial_{\xi}^{2}+1\right)\right) \partial_{\xi} \delta(\xi \pm \eta)}
\end{aligned}
$$




$$
\begin{aligned}
& {\left[\hat{T}_{\sigma}^{(+)}(\xi, t), \hat{J}_{n(r) \mu}^{( \pm)}(\eta, t, \sigma)\right]=\mp i \partial_{\eta}\left(\hat{J}_{n(r) \mu}^{( \pm)}(\eta, t, \sigma) \delta(\xi \mp \eta)\right)} \\
& {\left[\hat{T}_{\sigma}^{(-)}(\xi, t), \hat{J}_{n(r) \mu}^{( \pm)}(\eta, t, \sigma)\right]=\mp i \partial_{\eta}\left(\hat{J}_{n(r) \mu}^{( \pm)}(\eta, t, \sigma) \delta(\xi \pm \eta)\right)}
\end{aligned}
$$

follows from the commutators in Eqs. (2.1c), (2.1d). It is easily checked that this strip algebra is also locally twisted WZW, that is, locally isomorphic in the bulk to the corresponding rectified closed-string algebra (B.9).

In each sector $\sigma$ of $A_{g}^{\text {open }}(H) / H$, the Hamiltonian is constructed from the stress tensors by the usual integration:

$$
\begin{gathered}
\hat{H}_{\sigma}=\hat{H}_{\sigma}^{\dagger} \equiv L_{\sigma}(0)=\int_{0}^{\pi} d \xi\left(\hat{T}_{\sigma}^{(+)}(\xi, t)+\hat{T}_{\sigma}^{(-)}(\xi, t)\right)= \\
=\frac{1}{2 \pi} \int_{0}^{\pi} d \xi \mathcal{L}_{\hat{\mathfrak{g}}(\sigma)}^{n(r) \mu ;-n(r), \nu}(\sigma)\left(: \hat{J}_{n(r) \mu}^{(+)}(\xi, t, \sigma) \hat{J}_{-n(r), \nu}^{(+)}(\xi, t, \sigma)\right. \\
\left.+\hat{J}_{n(r) \mu}^{(-)}(\xi, t, \sigma) \hat{J}_{-n(r), \nu}^{(-)}(\xi, t, \sigma):\right) \\
\sigma=0, \ldots, N_{c}-1 .
\end{gathered}
$$

Then we have the open-string equations of motion

$$
\begin{aligned}
\partial_{t} \hat{A} & =i\left[\hat{H}_{\sigma}, \hat{A}\right] \\
\partial_{\mp} \hat{J}^{( \pm)}(\xi, t) & =\partial_{\mp} \hat{T}_{\sigma}^{( \pm)}(\xi, t)=0
\end{aligned}
$$

where the conservation laws in Eq. (2.16b) follow from Eqs. (2.16a) and (2.14).

We may also define the bulk momentum operator

$$
\begin{aligned}
& \hat{P}_{\sigma}=\hat{P}_{\sigma}^{\dagger} \equiv \int_{0}^{\pi} d \xi\left(\hat{T}_{\sigma}^{(+)}(\xi, t)-\hat{T}_{\sigma}^{(-)}(\xi, t)\right) \\
&=\frac{1}{2 \pi} \int_{0}^{\pi} d \xi \mathcal{L}_{\hat{\mathfrak{g}}(\sigma)}^{n(r) \mu ;-n(r), \nu}(\sigma)\left(: \hat{J}_{n(r) \mu}^{(+)}(\xi, t, \sigma) \hat{J}_{-n(r), \nu}^{(+)}(\xi, t, \sigma)\right. \\
&\left.\quad-\hat{J}_{n(r) \mu}^{(-)}(\xi, t, \sigma) \hat{J}_{-n(r), \nu}^{(-)}(\xi, t, \sigma):\right)
\end{aligned}
$$

in analogy to Ref. [23]. As in the untwisted case, this quantity is not conserved

$$
\frac{d}{d t} \hat{P}_{\sigma}=2\left(\hat{T}_{\sigma}^{(+)}(\pi)-\hat{T}_{\sigma}^{(+)}(0)\right) \neq 0 .
$$

and it can be checked with Eq. (2.14) that $\hat{P}_{\sigma}$ generates $-i \partial_{\xi}$ only in the bulk:

$$
i\left[\hat{P}_{\sigma}, \hat{J}_{n(r) \mu}^{( \pm)}(\xi, t, \sigma)\right]=\partial_{\xi} \begin{cases}\hat{J}_{n(r) \mu}^{( \pm)}(\xi, t, \sigma) & 0<\xi<\pi \\ 0 & \xi=0, \pi\end{cases}
$$


More generally, the correct form of $\partial_{\xi} \hat{A}$ for any $\hat{A}$ can be obtained from the form of $i\left[\hat{P}_{\sigma}, \hat{A}\right]$ in the bulk

$$
\partial_{\xi} \hat{A}=i\left[\hat{P}_{\sigma}, \hat{A}\right], \quad 0<\xi<\pi
$$

by smoothly extending this form to the boundary.

We conclude this section with a discussion of the scalar twist-field state $|0\rangle_{\sigma}$

$$
\hat{J}_{n(r) \mu}\left(m+\frac{n(r)}{\rho(\sigma)} \geq 0\right)|0\rangle_{\sigma}={ }_{\sigma}\langle 0| \hat{J}_{n(r) \mu}\left(m+\frac{n(r)}{\rho(\sigma)} \leq 0\right)=0, \quad \sigma=0, \ldots, N_{c}-1
$$

in sector $\sigma$ of $A_{g}^{\text {open }}(H) / H$. The conformal weight $\hat{\Delta}_{0}(\sigma)$ of the scalar twist-field

$$
\begin{gathered}
L_{\sigma}(m)^{\dagger}=L_{\sigma}(-m) \\
\left(L_{\sigma}(m \geq 0)-\delta_{m, 0} \hat{\Delta}_{0}(\sigma)\right)|0\rangle_{\sigma}={ }_{\sigma}\langle 0|\left(L_{\sigma}(m \leq 0)-\delta_{m, 0} \hat{\Delta}_{0}(\sigma)\right)=0 \\
\left(\hat{H}_{\sigma}-\hat{\Delta}_{0}(\sigma)\right)|0\rangle_{\sigma}={ }_{\sigma}\langle 0|\left(\hat{H}_{\sigma}-\hat{\Delta}_{0}(\sigma)\right)=0 \\
\hat{\Delta}_{0}(\sigma)=\frac{x_{\mathfrak{g}} / 2}{x_{\mathfrak{g}}+\tilde{h}_{\mathfrak{g}}} \sum_{r} \frac{\bar{n}(r)}{2 \rho(\sigma)}\left(1-\frac{\bar{n}(r)}{\rho(\sigma)}\right) \operatorname{dim}[\bar{n}(r)]
\end{gathered}
$$

follows from the defining relations (2.21) and Eq. (A.3a). The general formula for $\hat{\Delta}_{0}(\sigma)$ is given in Eq. (A.3e), while the simplified form given in Eq. (2.22d) holds when the underlying untwisted theory is permutation-invariant

$$
g=\oplus_{I} \mathfrak{g}^{I}, \quad \mathfrak{g}^{I} \simeq \text { simple } \mathfrak{g}, \quad k_{I}=k
$$

which includes all the basic types of WZW orbifolds. In Eq. (2.22), the quantities $\tilde{h}_{\mathfrak{g}}$ and $x_{\mathfrak{g}}$ are respectively the dual Coxeter number of $\mathfrak{g}$ and the invariant level of affine $\mathfrak{g}$, while $\operatorname{dim}[\bar{n}(r)]$ is the degeneracy of the eigenvalue $E_{n(r)}(\sigma)$ in the $H$-eigenvalue problem (2.2a). Further evaluation of $\hat{\Delta}_{0}(\sigma)$ is given for specific cases in Refs. [6, 7, , 9, 10].

\section{Classical Description of Twisted Open WZW Strings}

We turn next to the classical theory of the open-string WZW orbifold $A_{g}^{\text {open }}(H) / H$, beginning with the classical limit of the operator relations above. Using intuition gained from the classical results of this section, our discussion of the quantum theory will resume in Sec. 4.

\subsection{The Classical Strip Currents and Stress Tensors}

The classical (high-level) limit of the theory is obtained from the quantum theory above by dropping all normal ordering, omitting the central terms in Eq. (2.14) and carrying out 
the following replacements

$$
\begin{gathered}
{[,] \rightarrow\{,\}} \\
\mathcal{L}_{\hat{\mathfrak{g}}(\sigma)}^{n(r) \mu ; n(s) \nu}(\sigma) \underset{k \rightarrow \infty}{\longrightarrow} \frac{1}{2} \mathcal{G}^{n(r) \mu ; n(s) \nu}(\sigma)
\end{gathered}
$$

where $\{$,$\} are (rescaled) Poisson brackets. Here \mathcal{G}^{\bullet}(\sigma)$ is the inverse of the twisted tangentspace metric defined in Eq. (2.2a).

This gives for example the classical form of the open-string stress tensors

$$
\begin{gathered}
\hat{T}_{\sigma}^{( \pm)}(\xi, t) \equiv \frac{1}{4 \pi} \mathcal{G}^{n(r) \mu ;-n(r), \nu}(\sigma) \hat{J}_{n(r) \mu}^{( \pm)}(\xi, t, \sigma) \hat{J}_{-n(r), \nu}^{( \pm)}(\xi, t, \sigma), \quad 0 \leq \xi \leq \pi \\
\hat{T}_{\sigma}^{(+)}(\xi, t)=\hat{T}_{\sigma}^{(-)}(\xi, t) \text { at } \xi=0, \pi, \quad \partial_{\mp} \hat{T}_{\sigma}^{( \pm)}(\xi, t)=0
\end{gathered}
$$

where $\hat{J}^{( \pm)}$are now the classical strip currents. We list below the remainder of those classical relations which do not follow directly from the bracket substitution (3.1a) alone:

$$
\begin{gathered}
\left\{\hat{T}_{\sigma}^{(+)}(\xi, t), \hat{T}_{\sigma}^{( \pm)}(\eta, t)\right\}=i\left(\hat{T}_{\sigma}^{(+)}(\xi, t)+\hat{T}_{\sigma}^{( \pm)}(\eta, t)\right) \partial_{\xi} \delta(\xi \mp \eta) \\
\left\{\hat{T}_{\sigma}^{(-)}(\xi, t), \hat{T}_{\sigma}^{( \pm)}(\eta, t)\right\}=-i\left(\hat{T}_{\sigma}^{(-)}(\xi, t)+\hat{T}_{\sigma}^{( \pm)}(\eta, t)\right) \partial_{\xi} \delta(\xi \pm \eta) \\
\hat{H}_{\sigma}=\int_{0}^{\pi} d \xi\left(\hat{T}_{\sigma}^{(+)}(\xi, t)+\hat{T}_{\sigma}^{(-)}(\xi, t)\right) \\
=\frac{1}{4 \pi} \int_{0}^{\pi} d \xi \mathcal{G}^{n(r) \mu ;-n(r), \nu}(\sigma)\left(\hat{J}_{n(r) \mu}^{(+)}(\xi, t) \hat{J}_{-n(r), \nu}^{(+)}(\xi, t)+\hat{J}_{n(r) \mu}^{(-)}(\xi, t) \hat{J}_{-n(r), \nu}^{(-)}(\xi, t)\right) \\
\hat{P}_{\sigma}=\int_{0}^{\pi} d \xi\left(\hat{T}_{\sigma}^{(+)}(\xi, t)-\hat{T}_{\sigma}^{(-)}(\xi, t)\right) \\
=\frac{1}{4 \pi} \int_{0}^{\pi} d \xi \mathcal{G}^{n(r) \mu ;-n(r), \nu}(\sigma)\left(\hat{J}_{n(r) \mu}^{(+)}(\xi, t) \hat{J}_{-n(r), \nu}^{(+)}(\xi, t)-\hat{J}_{n(r) \mu}^{(-)}(\xi, t) \hat{J}_{-n(r), \nu}^{(-)}(\xi, t)\right)
\end{gathered}
$$

In particular, the equal-time current algebra (2.7) and the Hamiltonian equations of motion in Eqs. (2.16), (2.18) have the same form in the classical theory. 


\subsection{Phase-Space Realization of the Strip Currents}

To go beyond a theory of currents, we now postulate the following phase-space realization 23] of the twisted strip currents at time $t$ :

$$
\begin{gathered}
\hat{J}_{n(r) \mu}^{(+)}(\xi) \equiv \hat{J}_{n(r) \mu}^{(+)}(\xi, t, \sigma) \\
\equiv 2 \pi \hat{e}^{-1}(\hat{x}(\xi))_{n(r) \mu}{ }^{n(t) \delta} \hat{p}_{n(t) \delta}(\hat{B}, \xi)+\frac{1}{2} \partial_{\xi} \hat{x}_{\sigma}^{n(t) \delta}(\xi) \hat{e}(\hat{x}(\xi))_{n(t) \delta}{ }^{n(s) \nu} \mathcal{G}_{n(s) \nu ; n(r) \mu}(\sigma) \\
\hat{J}_{n(r) \mu}^{(-)}(\xi) \equiv \hat{J}_{n(r) \mu}^{(-)}(\xi, t, \sigma) \\
\equiv 2 \pi \hat{\bar{e}}^{-1}(\hat{x}(\xi))_{n(r) \mu}{ }^{n(t) \delta} \hat{p}_{n(t) \delta}(\hat{B}, \xi)-\frac{1}{2} \partial_{\xi} \hat{x}_{\sigma}^{n(t) \delta}(\xi) \hat{e}(\hat{x}(\xi))_{n(t) \delta}{ }^{n(s) \nu} \mathcal{G}_{n(s) \nu ; n(r) \mu}(\sigma) \\
\hat{p}_{n(r) \mu}(\hat{B}, \xi) \equiv \hat{p}_{n(r) \mu}^{\sigma}+\frac{1}{4 \pi} \hat{B}_{n(r) \mu ; n(s) \nu}(\hat{x}(\xi)) \partial_{\xi} \hat{x}_{\sigma}^{n(s) \nu}(\xi) \\
\sigma=0, \ldots, N_{c}-1 .
\end{gathered}
$$

Here $\left\{\hat{x}_{\sigma}\right\}$ is a set of Einstein coordinates and $\left\{\hat{p}^{\sigma}\right\}$ is a set of momenta which are expected to be canonically conjugate to $\hat{x}_{\sigma}$ in the bulk. The twisted vielbeins $\hat{e}, \hat{\bar{e}}$ and twisted B field $\hat{B}$ which appear in (3.5) are defined in terms of so-called group orbifold elements $\hat{g}$ :

$$
\begin{gathered}
\hat{g}(\mathcal{T}, \xi, t, \sigma)=e^{i \hat{x}_{\sigma}^{n(r) \mu}(\xi, t) \mathcal{T}_{n(r) \mu}(T, \sigma)} \\
\hat{e}_{n(r) \mu}(\mathcal{T}) \equiv-i \hat{g}^{-1}(\mathcal{T}) \hat{\partial}_{n(r) \mu} \hat{g}(\mathcal{T}) \equiv \hat{e}_{n(r) \mu}{ }^{n(s) \nu} \mathcal{T}_{n(s) \nu}, \quad \hat{\partial}_{n(r) \mu}(\xi) \equiv \frac{\partial}{\partial \hat{x}^{(r) \mu}(\xi)} \\
\hat{\bar{e}}_{n(r) \mu}(\mathcal{T}) \equiv-i \hat{g}(\mathcal{T}) \hat{\partial}_{n(r) \mu} \hat{g}^{-1}(\mathcal{T}) \equiv \hat{\bar{e}}_{n(r) \mu}{ }^{n(s) \nu} \mathcal{T}_{n(s) \nu} \\
\hat{e}_{n(r) \mu}{ }^{n(t) \delta} \hat{e}_{n(t) \delta}^{-1}{ }^{n(s) \nu}=\hat{\bar{e}}_{n(r) \mu}{ }^{n(t) \delta} \hat{\bar{e}}_{n(t) \delta}^{-1}{ }^{n(s) \nu}=\delta_{n(r) \mu}{ }^{n(s) \nu}=\delta_{\mu}^{\nu} \delta_{n(r)-n(s), 0 \bmod \rho(\sigma)} \\
\hat{H}_{n(r) \mu ; n(s) \nu ; n(t) \delta}(\hat{x}) \equiv \hat{\partial}_{n(r) \mu} \hat{B}_{n(s) \nu ; n(t) \delta}(\hat{x})+\hat{\partial}_{n(s) \nu} \hat{B}_{n(t) \delta ; n(r) \mu}(\hat{x})+\hat{\partial}_{n(t) \delta} \hat{B}_{n(r) \mu ; n(s) \nu}(\hat{x}) \\
=-i \widehat{T r}\left(\mathcal{M}\left(\mathcal{T}, \sigma \hat{e}_{n(r) \mu}(\mathcal{T}, \hat{x})\left[\hat{e}_{n(s) \nu}(\mathcal{T}, \hat{x}), \hat{e}_{n(t) \delta}(\mathcal{T}, \hat{x})\right]\right)\right. \\
=\hat{e}(\hat{x})_{n(r) \mu}^{n\left(r^{\prime}\right) \mu^{\prime}} \hat{e}(\hat{x})_{n(s) \nu}^{n\left(s^{\prime}\right) \nu^{\prime}} \hat{e}(\hat{x})_{n(t) \delta}^{n\left(t^{\prime}\right) \delta^{\prime}} \mathcal{F}_{n\left(r^{\prime}\right) \mu^{\prime} ; n\left(s^{\prime}\right) \nu^{\prime} ; n\left(t^{\prime}\right) \delta^{\prime}}(\sigma) .
\end{gathered}
$$

As in closed-string orbifold theory, the group orbifold element $\hat{g}(\mathcal{T}, \xi, t, \sigma)$ is locally a group element, where the group is generated by the orbifold Lie algebra (2.2fi) of twisted representation matrices $\mathcal{T}$. Moreover, we have chosen $\hat{e}(0)=\mathbb{1}, \hat{e}(0)=-\mathbb{1}$. The totally antisymmetric twisted structure constants $\mathcal{F}_{\bullet}(\sigma)$ in (3.6f) are constructed by lowering the last index of $\mathcal{F}(\sigma)$ using the twisted metric $\mathcal{G}_{\bullet}(\sigma)$.

Here we have generalized the strategy of Ref. 23] by modelling the realizations in Eq. (3.5) after the known phase-space realizations of $\hat{J}$ and $\hat{\bar{J}}$ in closed-string WZW orbifold 
theory [11], where the same geometric quantities and relations (3.6) also appear. However, as we will see below, the time dependence of these quantities in the open-string orbifolds is not the same as that in the closed-string orbifolds.

The phase-space form of the Hamiltonian

$$
\begin{gathered}
\hat{H}_{\sigma}=\int_{0}^{\pi} d \xi \hat{\mathcal{H}}_{\sigma}(\hat{x}(\xi), \hat{p}(\xi)), \quad \sigma=0, \ldots, N_{c}-1 \\
\hat{\mathcal{H}}_{\sigma}(\hat{x}(\xi), \hat{p}(\xi))=\frac{1}{4 \pi} \mathcal{G}^{n(r) \mu ; n(s) \nu}(\sigma)\left(\hat{J}_{n(r) \mu}^{(+)}(\xi, t) \hat{J}_{n(s) \nu}^{(+)}(\xi, t)+\hat{J}_{n(r) \mu}^{(-)}(\xi, t) \hat{J}_{n(s) \nu}^{(-)}(\xi, t)\right) \\
=2 \pi \hat{G}^{n(r) \mu ; n(s) \nu}(\hat{x}) \hat{p}_{n(r) \mu}(\hat{B}) \hat{p}_{n(s) \nu}(\hat{B})+\frac{1}{8 \pi} \partial_{\xi} \hat{x}_{\sigma}^{n(r) \mu} \partial_{\xi} \hat{x}_{\sigma}^{n(s) \nu} \hat{G}_{n(r) \mu ; n(s) \nu}(\hat{x}) \\
\hat{G}_{n(r) \mu ; n(s) \nu}(\hat{x}) \equiv \hat{e}_{n(r) \mu}{ }^{n(t) \delta} \hat{e}_{n(s) \nu}^{n(u) \epsilon} \mathcal{G}_{n(t) \delta ; n(u) \epsilon}(\sigma)=\hat{\bar{e}}_{n(r) \mu}{ }^{n(t) \delta} \hat{\bar{e}}_{n(s) \nu}^{n(u) \epsilon} \mathcal{G}_{n(t) \delta ; n(u) \epsilon}(\sigma) \\
\hat{G}^{n(r) \mu ; n(s) \nu}(\hat{x}) \equiv \mathcal{G}^{n(t) \delta ; n(u) \epsilon}(\sigma) \hat{e}_{n(t) \delta}^{-1}{ }^{n(r) \mu} \hat{e}_{n(u) \epsilon}^{-1}{ }^{n(s) \nu} \\
\hat{G}_{n(r) \mu ; n(t) \delta}(\hat{x}) \hat{G}^{n(t) \delta ; n(s) \nu}(\hat{x})=\delta_{n(r) \mu}^{n(s) \nu}
\end{gathered}
$$

follows from Eq. (3.4a) and the phase-space realization. In Eq. (3.7), the quantities $\hat{G}_{\bullet}$ and $\hat{G}^{\bullet}$ are the twisted Einstein metric and its inverse respectively. More general phase-space realizations of the currents, with the same Hamiltonian (i. e. T-dual formulations), will be discussed in a future paper.

As another application of the phase-space realization (3.5), we obtain the following boundary conditions in terms of the phase-space variables

$$
\begin{gathered}
4 \pi\left(_{\hat{e}_{n(r) \mu}^{-1}}^{n(s) \nu}-\hat{e}_{n(r) \mu}^{-1}{ }^{n(s) \nu}\right) \hat{p}_{n(s) \nu}(\hat{B})= \\
=\partial_{\xi} \hat{x}_{\sigma}^{n(s) \nu}\left(\hat{\bar{e}}_{n(s) \nu}^{n(t) \delta}+\hat{e}_{n(s) \nu}^{n(t) \delta}\right) \mathcal{G}_{n(t) \delta ; n(r) \mu}(\sigma) \text { at } \xi=0 \\
4 \pi\left(e^{-2 \pi i \frac{n(r)}{\rho(\sigma)}} \hat{\bar{e}}_{n(r) \mu}^{-1}{ }^{n(s) \nu}-\hat{e}_{n(r) \mu}^{-1}{ }^{n(s) \nu}\right) \hat{p}_{n(s) \nu}(\hat{B})= \\
=\partial_{\xi} \hat{x}_{\sigma}^{n(s) \nu}\left(e^{-2 \pi i \frac{n(r)}{\rho(\sigma)}} \hat{\bar{e}}_{n(s) \nu}^{n(t) \delta}+\hat{e}_{n(s) \nu}^{n(t) \delta}\right) \mathcal{G}_{n(t) \delta ; n(r) \mu}(\sigma) \text { at } \xi=\pi .
\end{gathered}
$$

from the current boundary conditions in Eq. (2.6).

\subsection{The Brackets of $\hat{J}^{( \pm)}$with $\hat{x}$}

In the method of Ref. 23], all phase-space brackets are obtained by solving partial differential equations derived from the current algebra and the postulated phase-space realization of the currents. 
Following this method, we begin our analysis of the open-string phase-space brackets by writing down the so-called inverse relations

$$
\begin{aligned}
\partial_{\xi} \hat{x}_{\sigma}^{n(r) \mu}(\xi)= & \hat{J}_{n(s) \nu}^{(+)}(\xi) \mathcal{G}^{n(s) \nu ; n(t) \delta}(\sigma) \hat{e}^{-1}(\xi)_{n(t) \delta}{ }^{n(r) \mu} \\
& -\hat{J}_{n(s) \nu}^{(-)}(\xi) \mathcal{G}^{n(s) \nu ; n(t) \delta}(\sigma) \hat{\bar{e}}^{-1}(\xi)_{n(t) \delta}{ }^{n(r) \mu}, \quad 0 \leq \xi \leq \pi \\
\hat{p}_{n(r) \mu}(\hat{B}, \xi) & =\frac{1}{4 \pi}\left(\hat{e}(\xi)_{n(r) \mu}^{n(s) \nu} \hat{J}_{n(s) \nu}^{(+)}(\xi)+\hat{e}(\xi)_{n(r) \mu}{ }^{n(s) \nu} \hat{J}_{n(s) \nu}^{(-)}(\xi)\right)
\end{aligned}
$$

which follow directly from the phase-space realization in Eq. (3.5)). We also give the spatial derivative of $\hat{g}$

$$
\begin{aligned}
& \partial_{\xi} \hat{g}(\mathcal{T}, \xi)=\partial_{\xi} \hat{x}_{\sigma}^{n(r) \mu} \hat{\partial}_{n(r) \mu} \hat{g}(\mathcal{T}, \xi)=i\left(\hat{g}(\mathcal{T}, \xi) \hat{J}^{(+)}(\mathcal{T}, \xi)+\hat{J}^{(-)}(\mathcal{T}, \xi) \hat{g}(\mathcal{T}, \xi)\right) \\
& \hat{J}^{( \pm)}(\mathcal{T}, \xi) \equiv \hat{J}_{n(r) \mu}^{( \pm)}(\xi) \mathcal{G}^{n(r) \mu ; n(s) \nu}(\sigma) \mathcal{T}_{n(s) \nu} \\
& \hat{J}_{n(r) \mu}^{( \pm)}(\xi)=\widehat{\operatorname{Tr}}\left(\mathcal{M}(\mathcal{T}, \sigma) \hat{J}^{( \pm)}(\mathcal{T}, \xi) \mathcal{T}_{n(r) \mu}\right)
\end{aligned}
$$

which follows via the chain rule from Eqs. (3.9) and (3.6).

In analogy with Eq. (4.3) of Ref. [23], we may now derive differential equations for the $\{\hat{J}, \hat{x}\}$ bracket

$$
\begin{aligned}
& \partial_{\eta}\left\{\hat{J}_{n(r) \mu}^{(+)}(\xi, t, \sigma), \hat{x}_{\sigma}^{n(s) \nu}(\eta, t)\right\}=\left\{\hat{J}_{n(r) \mu}^{(+)}(\xi, t, \sigma), \partial_{\eta} \hat{x}_{\sigma}^{n(s) \nu}(\eta, t)\right\} \\
& =\left\{\hat{J}^{(+)}(\xi), \hat{J}^{(+)}(\eta) \mathcal{G} \bullet(\sigma) \hat{e}^{-1}(\eta)-\hat{J}^{(-)}(\eta) \mathcal{G}^{\bullet}(\sigma) \hat{e}^{-1}(\eta)\right\} \\
& =2 \pi i\left(\mathcal{F}_{n(r) \mu ; n(t) \delta^{n(u) \epsilon}}(\sigma) \hat{J}_{n(u) \epsilon}^{(+)}(\eta)+\mathcal{G}_{n(r) \mu ; n(t) \delta}(\sigma) \partial_{\xi}\right) \times \\
& \times \delta_{\frac{n(r)}{\rho(\sigma)}}(\xi-\eta) \mathcal{G}^{n(t) \delta ; n(v) \kappa}(\sigma) \hat{e}^{-1}(\eta)_{n(v) \kappa}{ }^{n(s) \nu}
\end{aligned}
$$

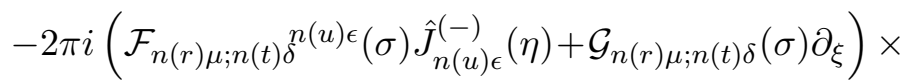

$$
\begin{aligned}
& \times \delta_{\frac{n(r)}{\rho(\sigma)}}(\xi+\eta) \mathcal{G}^{n(t) \delta ; n(v) \kappa}(\sigma) \hat{e}^{-1}(\eta)_{n(v) \kappa}{ }^{n(s) \nu} \\
& +\left\{\hat{J}_{n(r) \mu}^{(+)}(\xi), \hat{x}_{\sigma}^{n(t) \delta}(\eta)\right\}\left(\hat{\partial}_{n(t) \delta} \hat{e}^{-1}(\eta)_{n(u) \epsilon}{ }^{n(s) \nu} \mathcal{G}^{n(u) \epsilon ; n(v) \kappa}(\sigma) \hat{J}_{n(v) \kappa}^{(+)}(\eta)\right. \\
& \left.-\hat{\partial}_{n(t) \delta} \hat{e}^{-1}(\eta)_{n(u) \epsilon}{ }^{n(s) \nu} \mathcal{G}^{n(u) \epsilon ; n(v) \kappa}(\sigma) \hat{J}_{n(v) \kappa}^{(-)}(\eta)\right)
\end{aligned}
$$

where we have used the twisted current algebra (2.7), Eq. (3.9a) and the chain rule. The corresponding differential equation for the bracket $\left\{\hat{J}^{(-)}(\xi), \hat{x}(\eta)\right\}$ can be obtained by replacing $\xi \rightarrow-\xi$ everywhere in Eq. (3.11).

We wish to find a solution of these differential equations which is both locally twisted WZW and consistent with the strip boundary conditions for $\hat{J}^{( \pm)}(\xi)$ in Eq. (2.6). A natural 
candidate for the solution is:

$$
\begin{gathered}
\left\{\hat{J}_{n(r) \mu}^{(+)}(\xi, t, \sigma), \hat{x}_{\sigma}^{n(s) \nu}(\eta, t)\right\}= \\
-2 \pi i\left(\hat{e}^{-1}(\eta)_{n(r) \mu}^{n(s) \nu} \delta_{\frac{n(r)}{\rho(\sigma)}}(\xi-\eta)+\hat{\bar{e}}^{-1}(\eta)_{n(r) \mu}{ }^{n(s) \nu} \delta_{\frac{n(r)}{\rho(\sigma)}}(\xi+\eta)\right) \\
\left\{\hat{J}_{n(r) \mu}^{(-)}(\xi, t, \sigma), \hat{x}_{\sigma}^{n(s) \nu}(\eta, t)\right\}= \\
-2 \pi i\left(\hat{\bar{e}}^{-1}(\eta)_{n(r) \mu}^{n(s) \nu} \delta_{-\frac{n(r)}{\rho(\sigma)}}(\xi-\eta)+\hat{e}^{-1}(\eta)_{n(r) \mu}^{n(s) \nu} \delta_{-\frac{n(r)}{\rho(\sigma)}}(\xi+\eta)\right) \\
\sigma=0, \ldots, N_{c}-1 .
\end{gathered}
$$

According to Eqs. (B.13d,e) and the concluding paragraph of App. B, this candidate solution is locally twisted WZW. Moreover this candidate is consistent with the strip boundary conditions (2.6) because the relations

$$
\delta_{\frac{n(r)}{\rho(\sigma)}}(-\eta)=\delta_{-\frac{n(r)}{\rho(\sigma)}}(\eta), \quad \delta_{\frac{n(r)}{\rho(\sigma)}}(\pi \mp \eta)=e^{-2 \pi i \frac{n(r)}{\rho(\sigma)}} \delta_{-\frac{n(r)}{\rho(\sigma)}}(\pi \pm \eta)
$$

follow from the definition of the phase-modified delta functions.

Finally, one finds after considerable algebra that the candidate in Eq. (3.12) indeed solves the differential equations. To verify this, one inserts both Eq. (3.12) and the phasespace form (3.5) of $\hat{J}^{( \pm)}$into Eq. (3.11), following a line similar to the corresponding untwisted computation in Ref. 23]. In particular, the reader will find the following identities useful:

$$
\begin{aligned}
& \hat{\bar{e}}_{n(r) \mu}^{n(s) \nu}=-\hat{e}_{n(r) \mu}^{n(t) \delta} \hat{\Omega}_{n(t) \delta}^{n(s) \nu} \\
& \hat{e}_{n(r) \mu}^{-1}{ }^{n(u) \epsilon} \hat{\partial}_{n(u) \epsilon} \hat{\Omega}_{n(s) \nu}{ }^{n(t) \delta}=-\mathcal{F}_{n(r) \mu ; n(s) \nu}{ }^{n(u) \epsilon}(\sigma) \hat{\Omega}_{n(u) \epsilon}{ }^{n(t) \delta} \\
& \hat{\bar{e}}^{-1}{ }_{n(r) \mu}{ }^{n(u) \epsilon} \hat{\partial}_{n(u) \epsilon} \hat{\Omega}_{n(s) \nu}{ }^{n(t) \delta}=-\hat{\Omega}_{n(s) \nu}{ }^{n(u) \epsilon} \mathcal{F}_{n(u) \epsilon ; n(r) \mu}{ }^{n(t) \delta}(\sigma) \\
& \hat{\Omega}_{n(r) \mu}{ }^{n(t) \delta} \hat{\Omega}_{n(s) \nu}{ }^{n(u) \epsilon} \mathcal{G}_{n(t) \delta ; n(u) \epsilon}(\sigma)=\mathcal{G}_{n(r) \mu ; n(s) \nu}(\sigma) \\
& \hat{\Omega}_{n(r) \mu}{ }^{n(u) \epsilon} \hat{\Omega}_{n(s) \nu}{ }^{n(v) \kappa} \mathcal{F}_{n(u) \epsilon ; n(v) \kappa}{ }^{n(t) \delta}(\sigma)=\mathcal{F}_{n(r) \mu ; n(s) \nu}{ }^{n(u) \epsilon}(\sigma) \hat{\Omega}_{n(u) \epsilon}{ }^{n(t) \delta} \\
& \hat{\bar{e}}_{n(r) \mu}{ }^{n(u) \epsilon} \hat{\bar{e}}_{n(s) \nu}{ }^{n(v) \kappa} \mathcal{F}_{n(u) \epsilon ; n(v) \kappa}{ }^{n(w) \lambda}(\sigma) \hat{\bar{e}}^{-1}{ }_{n(w) \lambda} n(t) \delta \\
& =-\hat{e}_{n(r) \mu}{ }^{n(u) \epsilon} \hat{e}_{n(s) \nu}^{n(v) \kappa} \mathcal{F}_{n(u) \epsilon ; n(v) \kappa}{ }^{n(w) \lambda}(\sigma) \hat{e}_{n(w) \lambda}^{-1}{ }^{n(t) \delta} \\
& \hat{\partial}_{n(r) \mu} \hat{e}_{n(s) \nu}^{n(t) \delta}-\hat{\partial}_{n(s) \nu} \hat{e}_{n(r) \mu}^{n(t) \delta}=\hat{e}_{n(r) \mu}{ }^{n(u) \epsilon} \hat{e}_{n(s) \nu}{ }^{n(v) \kappa} \mathcal{F}_{n(u) \epsilon ; n(v) \kappa}{ }^{n(t) \delta}(\sigma) \\
& \hat{e}_{n(r) \mu}^{-1}{ }^{n(t) \delta} \hat{\partial}_{n(t) \delta} \hat{e}_{n(s) \nu}^{-1}{ }^{n(u) \epsilon}-\hat{e}_{n(s) \nu}^{-1}{ }^{n(t) \delta} \hat{\partial}_{n(t) \delta} \hat{e}_{n(r) \mu}^{-1}{ }^{n(u) \epsilon}=\mathcal{F}_{n(s) \nu ; n(r) \mu}{ }^{n(t) \delta}(\sigma) \hat{e}_{n(t) \delta}^{-1}{ }^{n(u) \epsilon} \text {. }
\end{aligned}
$$


In this list, the quantity $\hat{\Omega}$ is the twisted adjoint action

$$
\hat{g}(\mathcal{T}, \xi, t, \sigma) \mathcal{T}_{n(r) \mu} \hat{g}^{-1}(\mathcal{T}, \xi, t, \sigma) \equiv \hat{\Omega}(\hat{x}(\xi))_{n(r) \mu}{ }^{n(s) \nu} \mathcal{T}_{n(s) \nu}
$$

and the relations $(3.14 \mathrm{~g}, \mathrm{~h})$ are respectively the twisted Cartan-Maurer and inverse twisted Cartan-Maurer identities (the CM identities also hold for $\hat{e} \rightarrow \hat{\bar{e}}$ ). These identities and others of this type also appear in the geometry [11] of closed-string WZW orbifolds.

The general solution of the inhomogeneous differential equations contains an additional term which satisfies the corresponding homogeneous equations, but (as in Ref. [23]) one finds that such a term would violate the requirement that the strip algebra is locally twisted WZW.

In the solution (3.12), the extra boundary terms proportional to $\delta_{ \pm \frac{n(r)}{\rho(\sigma)}}(\xi+\eta)$ can be interpreted with Ref. [23] as the interaction of a non-abelian charge at $\xi$ with a non-abelian image charge at $-\eta$.

As an application of the $\{\hat{J}, \hat{x}\}$ brackets, we may now use the chain rule and Eqs. $(3.6 \mathrm{~b}, \mathrm{c})$ to obtain the brackets of the twisted strip currents with the group orbifold elements

$$
\begin{aligned}
\left\{\hat{J}_{n(r) \mu}^{(+)}(\xi, t, \sigma), \hat{g}(\mathcal{T}, \eta, t, \sigma)\right\}=2 \pi( & \left(\hat{g}(\mathcal{T}, \eta) \mathcal{T}_{n(r) \mu} \delta_{\frac{n(r)}{\rho(\sigma)}}(\xi-\eta)\right. \\
& \left.-\mathcal{T}_{n(r) \mu} \hat{g}(\mathcal{T}, \eta) \delta_{\frac{n(r)}{\rho(\sigma)}}(\xi+\eta)\right) \\
\left\{\hat{J}_{n(r) \mu}^{(-)}(\xi, t, \sigma), \hat{g}(\mathcal{T}, \eta, t, \sigma)\right\}=2 \pi( & -\mathcal{T}_{n(r) \mu} \hat{g}(\mathcal{T}, \eta) \delta_{-\frac{n(r)}{\rho(\sigma)}}(\xi-\eta) \\
& \left.+\hat{g}(\mathcal{T}, \eta) \mathcal{T}_{n(r) \mu} \delta_{-\frac{n(r)}{\rho(\sigma)}}(\xi+\eta)\right)
\end{aligned}
$$

and we have checked with Eqs. (2.7) and (2.2f) that all $\hat{J}, \hat{J}, \hat{g}$ bracket Jacobi identities for this algebra are satisfied. We note in App. B that the result (3.16) is also locally twisted WZW, that is, isomorphic in the bulk to the corresponding rectified WZW orbifold result (B.14).

The result (3.16) is easily quantized because it is linear in $\hat{g}$, and the operator form of this result will play a central role in our discussion of the quantum theory of open-string WZW orbifolds (see Sec. 4).

Finally, using the integral identities (C.3) and the $\{\hat{J}, \hat{g}\}$ brackets in Eq. (3.16), we may 
also compute the action of the bulk momentum (3.4b) on the group orbifold elements

$$
\begin{aligned}
& i\left\{\hat{P}_{\sigma}(t), \hat{g}(\mathcal{T}, \xi, t)\right\}= \\
& \quad= \begin{cases}i\left(\hat{g}(\mathcal{T}, \xi, t) \hat{J}^{(+)}(\mathcal{T}, \xi, t)+\hat{J}^{(-)}(\mathcal{T}, \xi, t) \hat{g}(\mathcal{T}, \xi, t)\right) & \text { for } 0<\xi<\pi \\
0 & \text { for } \xi=0, \pi\end{cases}
\end{aligned}
$$

and we note that this result agrees in the bulk with the form of $\partial_{\xi} \hat{g}$ given in Eq. (3.10a).

\subsection{Coordinate Space}

We now move to coordinate space, beginning with the computation of $\partial_{t} \hat{x}_{\sigma}$ :

$$
\begin{aligned}
\partial_{t} \hat{x}_{\sigma}^{n(r) \mu}(\xi, t) & =i\left\{\hat{H}_{\sigma}(t), \hat{x}_{\sigma}^{n(r) \mu}(\xi, t)\right\} \\
& =\mathcal{G}^{n(s) \nu ; n(t) \delta}(\sigma)\left(\hat{e}^{-1}(\xi)_{n(s) \nu}^{n(r) \mu} \hat{J}_{n(t) \delta}^{(+)}(\xi)+\hat{\bar{e}}^{-1}(\xi)_{n(s) \nu}{ }^{n(r) \mu} \hat{J}_{n(t) \delta}^{(-)}(\xi)\right) \\
& =4 \pi \hat{G}^{n(r) \mu ; n(s) \nu}(\hat{x}(\xi, t)) \hat{p}_{n(s) \nu}(\hat{B}, \xi, t) \\
\hat{p}_{n(r) \mu}(\hat{B}, \xi, t) & =\frac{1}{4 \pi} \hat{G}_{n(r) \mu ; n(s) \nu}(\hat{x}(\xi, t)) \partial_{t} \hat{x}_{\sigma}^{n(s) \nu}(\xi, t) .
\end{aligned}
$$

Here we have used the form of the Hamiltonian in Eq. (3.7) and the $\{\hat{J}, \hat{x}\}$ brackets (3.12), as well as Eqs. (C.3), (3.7c) and (3.9b). These relations between $\partial_{t} \hat{x}_{\sigma}$ and $\hat{p}(\hat{B})$ are locally twisted WZW, i. e. they are the same as those found in closed-string WZW orbifold theory [11. This is not surprising because the locally twisted WZW requirement also played a role in determining the form (3.12) of the $\{\hat{J}, \hat{x}\}$ brackets $^{\ddagger 3}$, which were used in this computation.

As an application of the result (3.18a), we use the chain rule and Eq. (3.6) to derive an equation for the time derivative of the group orbifold element

$$
\begin{array}{r}
\partial_{t} \hat{g}(\mathcal{T}, \xi, t, \sigma)=i\left(\hat{g}(\mathcal{T}, \xi, t, \sigma) \hat{J}^{(+)}(\mathcal{T}, \xi, t, \sigma)-\hat{J}^{(-)}(\mathcal{T}, \xi, t, \sigma) \hat{g}(\mathcal{T}, \xi, t, \sigma)\right) \\
\partial_{\xi} \hat{g}(\mathcal{T}, \xi, t, \sigma)=i\left(\hat{g}(\mathcal{T}, \xi, t, \sigma) \hat{J}^{(+)}(\mathcal{T}, \xi, t, \sigma)+\hat{J}^{(-)}(\mathcal{T}, \xi, t, \sigma) \hat{g}(\mathcal{T}, \xi, t, \sigma)\right) \\
\partial_{+} \hat{g}(\mathcal{T}, \xi, t, \sigma)=2 i \hat{g}(\mathcal{T}, \xi, t . \sigma) \hat{J}^{(+)}(\mathcal{T}, \xi, t, \sigma) \\
\partial_{-} \hat{g}(\mathcal{T}, \xi, t, \sigma)=-2 i \hat{J}^{(-)}(\mathcal{T}, \xi, t, \sigma) \hat{g}(\mathcal{T}, \xi, t, \sigma)
\end{array}
$$

where the matrix currents $\hat{J}^{( \pm)}(\mathcal{T})$ are defined in Eq. (3.10b). For symmetry, we have also included the corresponding spatial derivative (3.10a of $\hat{g}$. Note that these equations of

\footnotetext{
${ }^{\ddagger 3}$ In fact, the locally twisted WZW property of the results (3.18) tells us that our solution (3.12) for $\{\hat{J}, \hat{x}\}$ is a posteriori correct - even without consideration of the rectification problem (see App. B).
} 
motion are the same as those of closed-string WZW orbifold theory [6, 11] and hence are locally twisted WZW.

Similarly, we may substitute Eq. (3.18c) into the phase-space realization (3.5) to obtain the coordinate-space form of the twisted strip currents:

$$
\begin{gathered}
\hat{J}_{n(r) \mu}^{(+)}(\xi, t, \sigma)=\frac{1}{2} \partial_{+} \hat{x}_{\sigma}^{n(s) \nu}(\xi, t) \hat{e}(\xi, t)_{n(s) \nu}{ }^{n(t) \delta} \mathcal{G}_{n(t) \delta ; n(r) \mu}(\sigma) \\
\hat{J}_{n(r) \mu}^{(-)}(\xi, t, \sigma)=\frac{1}{2} \partial_{-} \hat{x}_{\sigma}^{n(s) \nu}(\xi, t) \hat{e}(\xi, t)_{n(s) \nu}{ }^{n(t) \delta} \mathcal{G}_{n(t) \delta ; n(r) \mu}(\sigma) \\
\hat{J}^{(+)}(\mathcal{T}, \xi, t, \sigma)=-\frac{i}{2} \hat{g}^{-1}(\mathcal{T}, \xi, t, \sigma) \partial_{+} \hat{g}(\mathcal{T}, \xi, t, \sigma) \\
\hat{J}^{(-)}(\mathcal{T}, \xi, t, \sigma)=-\frac{i}{2} \hat{g}(\mathcal{T}, \xi, t, \sigma) \partial_{-} \hat{g}^{-1}(\mathcal{T}, \xi, t, \sigma) \\
\partial_{-}\left(\hat{g}^{-1}(\mathcal{T}) \partial_{+} \hat{g}(\mathcal{T})\right)=\partial_{+}\left(\hat{g}(\mathcal{T}) \partial_{-} \hat{g}^{-1}(\mathcal{T})\right)=0
\end{gathered}
$$

The relations in Eqs. (3.20c,d) may also be obtained by inverting Eqs. (3.19c,d). The results in Eq. (3.20e) follow from Eqs. (3.20c,d) and the conservation (2.5c) of the currents.

We may also define a bulk Lagrange density for each twisted open WZW string by the usual Legendre transformation

$$
\begin{aligned}
& \hat{\mathcal{L}}_{\sigma} \equiv \partial_{t} \hat{x}_{\sigma}^{n(r) \mu} \hat{p}_{n(r) \mu}-\hat{\mathcal{H}}_{\sigma} \\
&=\frac{1}{8 \pi}\left(\hat{G}_{n(r) \mu ; n(s) \nu}+\hat{B}_{n(r) \mu ; n(s) \nu}\right) \partial_{+} \hat{x}_{\sigma}^{n(r) \mu} \partial_{-} \hat{x}_{\sigma}^{n(s) \nu}, \quad 0<\xi<\pi \\
& \frac{1}{8 \pi} \hat{G}_{n(r) \mu ; n(s) \nu} \partial_{+} \hat{x}_{\sigma}^{n(r) \mu} \partial_{-} \hat{x}_{\sigma}^{n(s) \nu}=-\frac{1}{8 \pi} \widehat{\operatorname{Tr}}\left(\mathcal{M}(\mathcal{T}, \sigma) \hat{g}^{-1}(\mathcal{T}) \partial_{+} \hat{g}(\mathcal{T}) \hat{g}^{-1}(\mathcal{T}) \partial_{-} \hat{g}(\mathcal{T})\right)
\end{aligned}
$$

where $\mathcal{H}_{\sigma}$ is the Hamiltonian density in Eq. (3.7b), and $\mathcal{M}(\mathcal{T}, \sigma)$ in Eq. (3.21b) is the twisted data matrix encountered in Eq. (2.2g). As expected, this Lagrange density is locally twisted WZW, i. e. isomorphic in the bulk to the twisted sigma model form of the closed-string WZW orbifold Lagrange density [1].

A complete action formulation of untwisted open WZW strings (in terms of group elements and extra boundary variables) was given in Ref. 40], but the complete action formulation of the basic class of twisted open WZW strings is an open problem. We note in passing, however, that the complete action formulation is in fact known for the twisted open-string sectors of the WZW orientation orbifolds [17. This action is expressed in terms of group orbifold elements alone on the solid half cylinder, but we remind the reader that the open-string orientation orbifold sectors are not included in the basic class of twisted open strings. 


\subsection{The Generalized WZW Branes of $A_{g}^{\text {open }}(H) / H$}

Using Eq. (3.18c), we find that the phase-space form (3.8) of the boundary conditions can be written in the following two coordinate-space forms

$$
\begin{aligned}
& \hat{J}_{n(r) \mu}^{(+)}(0, t, \sigma)=\hat{J}_{n(r) \mu}^{(-)}(0, t, \sigma), \quad \hat{J}_{n(r) \mu}^{(+)}(\pi, t, \sigma)=e^{-2 \pi i \frac{n(r)}{\rho(\sigma)}} \hat{J}_{n(r) \mu}^{(-)}(\pi, t, \sigma) \\
& \partial_{t} \hat{x}_{\sigma}^{n(r) \mu}(\xi, t)\left(\hat{\bar{e}}(\xi, t)_{n(r) \mu}{ }^{n(s) \nu}-\hat{e}(\xi, t)_{n(r) \mu}{ }^{n(s) \nu}\right) \\
& =\partial_{\xi} \hat{x}^{n(r) \mu}(\xi, t)\left(\hat{\bar{e}}(\xi, t)_{n(r) \mu}^{n(s) \nu}+\hat{e}(\xi, t)_{n(r) \mu}^{n(s) \nu}\right) \text { at } \xi=0 \\
& \partial_{t} \hat{x}_{\sigma}^{n(r) \mu}(\xi, t)\left(e^{2 \pi i \frac{n(s)}{\rho(\sigma)}} \hat{\bar{e}}(\xi, t)_{n(r) \mu}^{n(s) \nu}-\hat{e}(\xi, t)_{n(r) \mu}^{n(s) \nu}\right) \\
& =\partial_{\xi} \hat{x}^{n(r) \mu}(\xi, t)\left(e^{2 \pi i \frac{n(s)}{\rho(\sigma)}} \hat{e}(\xi, t)_{n(r) \mu}{ }^{n(s) \nu}+\hat{e}(\xi, t)_{n(r) \mu}{ }^{n(s) \nu}\right) \text { at } \xi=\pi
\end{aligned}
$$

where the form in Eq. (3.22) appeared earlier in Eq. (2.6). The equivalence of Eqs. (3.22) and (3.23) also follows directly from Eq. (3.20).

These coordinate-space boundary conditions can be reexpressed in terms of the matrix currents and/or the group orbifold elements:

$$
\begin{gathered}
\hat{J}^{(+)}(\mathcal{T}, 0, t, \sigma)=\hat{J}^{(-)}(\mathcal{T}, 0, t, \sigma), \quad \hat{J}^{(+)}(\mathcal{T}, \pi, t, \sigma)=E(T, \sigma) \hat{J}^{(-)}(\mathcal{T}, \pi, t, \sigma) E(T, \sigma)^{*} \\
\hat{g}^{-1}(\mathcal{T}, \xi, t, \sigma) \partial_{+} \hat{g}(\mathcal{T}, \xi, t, \sigma) \\
= \begin{cases}\hat{g}(\mathcal{T}, \xi, t, \sigma) \partial_{-} \hat{g}^{-1}(\mathcal{T}, \xi, t, \sigma) & \text { at } \xi=0 \\
E(T, \sigma) \hat{g}(\mathcal{T}, \xi, t, \sigma) \partial_{-} \hat{g}^{-1}(\mathcal{T}, \xi, t, \sigma) E(T, \sigma)^{*} & \text { at } \xi=\pi \\
\sigma=0, \ldots, N_{c}-1 .\end{cases}
\end{gathered}
$$

These results follow from Eqs. (3.10b), (3.20c,d), (3.22) and the selection rule (2.2el) for the twisted representation matrices. We remind that $E(T, \sigma)$ is the eigenvalue matrix of the extended $H$-eigenvalue problem in Eq. (2.3).

The first part of Eq. (3.24b) tells us that each twisted open WZW string ends at $\xi=0$ on an ordinary WZW brane [39, 23]. The second part of Eq. (3.24b) tells us however that the open string ends at $\xi=\pi$ on what may be called a generalized WZW brane-different 
from the ordinary WZW brane at $\xi=0$. Indeed, the twisting of our open WZW strings can be understood as a consequence of having different branes at each end of the string ${ }^{\ddagger 4}$.

We also give the form of the generalized WZW branes in terms of matrix elements

$$
\begin{aligned}
\left(\hat{g}^{-1}(\mathcal{T}, \xi, t, \sigma) \partial_{+} \hat{g}(\mathcal{T}, \xi, t, \sigma)\right)_{N(r) \mu}^{N(s) \nu} \\
\quad= \begin{cases}\left(\hat{g}(\mathcal{T}, \xi, t, \sigma) \partial_{-} \hat{g}^{-1}(\mathcal{T}, \xi, t, \sigma)\right)_{N(r) \mu}^{N(s) \nu} & \text { at } \xi=0 \\
e^{-2 \pi i \frac{N(r)-N(s)}{R(\sigma)}}\left(\hat{g}(\mathcal{T}, \xi, t, \sigma) \partial_{-} \hat{g}^{-1}(\mathcal{T}, \xi, t, \sigma)\right)_{N(r) \mu} N(s) \nu & \text { at } \xi=\pi\end{cases}
\end{aligned}
$$

where we have used the form of $E(T, \sigma)$ in Eq. (2.3) and the fact that the index structure of $\hat{g}(\mathcal{T})$ is the same as that of $\mathcal{T}$.

The description of the generalized WZW branes in Eqs. (3.24), (3.25) is a central result of this paper.

As an explicit example, we give the matrix element form of the branes in sector $\sigma$ of the general open-string WZW permutation orbifold:

$$
\begin{aligned}
& n(r) \mu \rightarrow \hat{j} a j, \quad N(r) \mu \rightarrow \hat{j} \alpha j, \quad \frac{n(r)}{\rho(\sigma)}=\frac{N(r)}{R(\sigma)}=\frac{\hat{j}}{f_{j}(\sigma)} \\
& \mathcal{T}_{\hat{j} a j}(T, \sigma)=T_{a} t_{\hat{j} j}(\sigma), \quad t_{\hat{j} j}(\sigma)_{\hat{l} l}^{\hat{m} m}=\delta_{j l} \delta_{l}^{m} \delta_{\hat{j}+\hat{l}-\hat{m}, 0 \bmod f_{j}(\sigma)} \\
& {\left[T_{a}, T_{b}\right]=i f_{a b}{ }^{c} T_{c}, \quad t_{\hat{j} j}(\sigma) t_{\hat{l l}}(\sigma)=\delta_{j l} t_{\hat{j}+\hat{l}, j}(\sigma)} \\
& \hat{g}(\mathcal{T}, \xi, t, \sigma)_{\hat{j} \alpha j}{ }^{\hat{l} \beta l}=\delta_{j}{ }^{l} \hat{g}_{j}(\mathcal{T}, \xi, t, \sigma)_{\hat{j} \alpha}{ }^{\hat{l} \beta} \\
& \left(\hat{g}^{-1}(\mathcal{T}, \xi, t, \sigma) \partial_{+} \hat{g}(\mathcal{T}, \xi, t, \sigma)\right)_{\hat{j} \alpha j}{ }^{\hat{l} \beta l}=\delta_{j}{ }^{l}\left(\hat{g}_{j}^{-1}(\mathcal{T}, \xi, t, \sigma) \partial_{+} \hat{g}_{j}(\mathcal{T}, \xi, t, \sigma)\right)_{\hat{j} \alpha}{ }^{\hat{l} \beta} \\
& =\delta_{j}{ }^{l} \begin{cases}\left(\hat{g}_{j}(\mathcal{T}, \xi, t, \sigma) \partial_{-} \hat{g}_{j}^{-1}(\mathcal{T}, \xi, t, \sigma)\right)_{\hat{j} \alpha} \hat{\imath} \beta & \text { at } \xi=0 \\
e^{-2 \pi i \frac{\hat{j}-\hat{l}}{f_{j}(\sigma)}}\left(\hat{g}_{j}(\mathcal{T}, \xi, t, \sigma) \partial_{-} \hat{g}_{j}^{-1}(\mathcal{T}, \xi, t, \sigma)\right)_{\hat{j} \alpha} \hat{l}^{\hat{l} \beta} & \text { at } \xi=\pi\end{cases} \\
& g=\oplus_{I} \mathfrak{g}^{I}, \quad \mathfrak{g}^{I} \simeq \mathfrak{g}, \quad T^{I} \simeq T \\
& a=1, \ldots, \operatorname{dim} \mathfrak{g}, \quad \alpha=1, \ldots, \operatorname{dim} T, \overline{\hat{j}}=0, \ldots, f_{j}(\sigma)-1, \sigma=0, \ldots, N_{c}-1 .
\end{aligned}
$$

Here $T$ is any irrep of Lie $\mathfrak{g}$ which labels an integrable irrep at level $k$ of affine $\mathfrak{g}$. This twisted open string is obtained when we appropriate our initial data from sector $\sigma$ of the general closed-string WZW permutation orbifold [6, 7, 9] on semisimple $g$. In this case, the

\footnotetext{
$\ddagger^{4}$ The prototype of this situation - a twisted open string with Neumann boundary conditions at one end and Dirichlet at the other - was first studied by Siegel in Ref. [41].
} 
twisted current algebra of sector $\sigma$

$$
\begin{aligned}
{\left[\hat{J}_{\hat{j} a j}\left(m+\frac{\hat{j}}{f_{j}(\sigma)}\right), \hat{J}_{\hat{l} b l}\left(n+\frac{\hat{l}}{f_{l}(\sigma)}\right)\right]=} & \delta_{j l}\left(i f_{a b}{ }^{c} \hat{J}_{\hat{j}+\hat{l}, c j}\left(m+n+\frac{\hat{j}+\hat{l}}{f_{j}(\sigma)}\right)+\right. \\
& \left.+\eta_{a b} k f_{j}(\sigma)\left(m+\frac{\hat{j}}{f_{j}(\sigma)}\right) \delta_{m+n+\frac{\hat{j}+\hat{l}}{f_{j}(\sigma)}, 0}\right)
\end{aligned}
$$

is known as a general orbifold affine algebra [1,3,5-7,9]. The results (3.26), (3.27) are given in the cycle notation for permutation orbifolds [7, 9], where $f_{j}(\sigma)$ is the length of cycle $j$ and $\hat{j}$ labels the position in each cycle, e. g. :

$$
\begin{aligned}
& \mathbb{Z}_{\lambda}: f_{j}(\sigma)=\rho(\sigma), \quad \overline{\hat{j}}=0, \ldots, \rho(\sigma)-1, j=0, \ldots, \frac{\lambda}{\rho(\sigma)}-1, \sigma=0, \ldots, \rho(\sigma)-1 \\
& \mathbb{Z}_{\lambda}, \lambda=\text { prime }: \quad \rho(\sigma)=\lambda, \quad \overline{\hat{j}}=0, \ldots, \lambda-1, \quad j=0, \quad \sigma=1, \ldots, \lambda-1 \\
& S_{N}: \quad f_{j}(\sigma)=\sigma_{j}, \quad \sigma_{j+1} \leq \sigma_{j}, \quad j=0, \ldots, n(\vec{\sigma})-1, \quad \sum_{j=0}^{n(\vec{\sigma})-1} \sigma_{j}=N .
\end{aligned}
$$

The cycle-diagonal form of the group orbifold element $\hat{g}$ in Eq. (3.26d) follows as in Ref. 9] from the form of $\mathcal{T}$ in Eq. (3.26b) and the exponential form (3.6a).

Similarly, the explicit data [6, 7, 9, 10, for the various closed-string orbifolds on simple $g$ can be substituted in Eq. (3.24b) to obtain the branes of the corresponding open-string WZW orbifolds.

\subsection{The Non-Commutative Geometry of $A_{g}^{\text {open }}(H) / H$}

In this subsection, we continue our phase-space construction to determine the equal-time coordinate brackets

$$
\hat{\Delta}_{\sigma}^{n(r) \mu ; n(s) \nu}(\xi, \eta) \equiv \hat{\Delta}^{n(r) \mu ; n(s) \nu}(\xi, \eta, t, \sigma)=\left\{\hat{x}_{\sigma}^{n(r) \mu}(\xi, t), \hat{x}_{\sigma}^{n(s) \nu}(\eta, t)\right\}
$$

which will generalize the open-string non-commutative geometry of Ref. [23].

To begin, we use the inverse relations (3.9) and the $\{\hat{J}, \hat{x}\}$ brackets (3.12) to find the following partial differential equation for $\hat{\Delta}_{\sigma}$ :

$$
\begin{aligned}
\partial_{\eta} & \hat{\Delta}_{\sigma}^{n(r) \mu ; n(s) \nu}(\xi, \eta) \\
= & 2 \pi i \mathcal{G}^{n(t) \delta ; n(u) \epsilon}(\sigma)\left(\hat{\bar{e}}^{-1}(\xi)_{n(t) \delta} \delta^{n(r) \mu} \hat{e}^{-1}(\eta)_{n(u) \epsilon} n(s) \nu\right. \\
& \left.\quad-\hat{e}^{-1}(\xi)_{n(u) \epsilon}^{n(r) \mu} \hat{\bar{e}}^{-1}(\eta)_{n(t) \delta} n(s) \nu\right) \delta_{\frac{n(t)}{\rho(\sigma)}}(\xi+\eta)+\hat{\Delta}_{\sigma}^{n(r) \mu ; n(t) \delta}(\xi, \eta) \hat{\Lambda}_{\sigma}(\eta)_{n(t) \delta} n(s) \nu \\
= & 2 \pi i \hat{\Psi}_{\sigma}^{n(r) \mu ; n(s) \nu}(\xi, \eta) \delta(\xi+\eta)+\hat{\Delta}_{\sigma}^{n(r) \mu ; n(t) \delta}(\xi, \eta) \hat{\Lambda}_{\sigma}(\eta)_{n(t) \delta}^{n(s) \nu}
\end{aligned}
$$




$$
\begin{aligned}
& \hat{\Psi}_{\sigma}^{n(r) \mu ; n(s) \nu}(\xi, \eta) \equiv \hat{\Psi}^{n(r) \mu ; n(s) \nu}(\xi, \eta, t, \sigma) \\
&=\mathcal{G}^{n(t) \delta ; n(u) \epsilon}(\sigma) e^{-i \frac{n(t)}{\rho(\sigma)}(\xi+\eta)} \times \\
& \\
&\left.\times\left(\hat{\bar{e}}^{-1}(\xi)_{n(t) \delta}^{n(r) \mu} \hat{e}^{-1}(\eta)_{n(u) \epsilon}{ }^{n(s) \nu}-\hat{e}^{-1}(\xi)_{n(u) \epsilon}\right)^{n(r) \mu} \hat{\bar{e}}^{-1}(\eta)_{n(t) \delta}^{n(s) \nu}\right) \\
& \hat{\Lambda}_{\sigma}(\eta)_{n(t) \delta}^{n(s) \nu} \equiv \\
&=\mathcal{G}^{n(u) \epsilon ; n(v) \kappa}(\sigma)\left(\hat{J}_{n(u) \epsilon}^{(+)}(\eta) \hat{\partial}_{n(t) \delta} \hat{e}^{-1}(\eta)_{n(v) \kappa}^{n(s) \nu}-\hat{J}_{n(u) \epsilon}^{(-)}(\eta) \hat{\partial}_{n(t) \delta} \hat{\bar{e}}^{-1}(\eta)_{n(v) \kappa}^{n(s) \nu}\right) .
\end{aligned}
$$

The corresponding $\partial_{\eta} \hat{\Delta}_{\sigma}$ equation is obtained from this result by noting that the bracket $\hat{\Delta}_{\sigma}$ is totally anti-symmetric under the exchange $\xi \leftrightarrow \eta, n(r) \mu \leftrightarrow n(s) \nu$.

In a compact matrix notation [23], these PDEs read

$$
\begin{gathered}
\partial_{\eta} \hat{\Delta}_{\sigma}(\xi, \eta)=2 \pi i \hat{\Psi}_{\sigma}(\xi, \eta) \delta(\xi+\eta)+\hat{\Delta}_{\sigma}(\xi, \eta) \hat{\Lambda}_{\sigma}(\eta) \\
\partial_{\xi} \hat{\Delta}_{\sigma}(\xi, \eta)=2 \pi i \hat{\Psi}_{\sigma}(\xi, \eta) \delta(\xi+\eta)+\hat{\Lambda}_{\sigma}^{t}(\xi) \hat{\Delta}_{\sigma}(\xi, \eta) \\
\hat{\Psi}_{\sigma}^{t}(\eta, \xi)=-\hat{\Psi}_{\sigma}(\xi, \eta)
\end{gathered}
$$

where $t$ is matrix transpose. The integrability condition for this system is:

$$
\begin{gathered}
\partial_{\xi} \partial_{\eta} \hat{\Delta}_{\sigma}(\xi, \eta)=\partial_{\eta} \partial_{\xi} \hat{\Delta}_{\sigma}(\xi, \eta) \text { iff } \\
\left(\partial_{\eta} \hat{\Psi}_{\sigma}(\xi, \eta)-\hat{\Psi}_{\sigma}(\xi, \eta) \hat{\Lambda}_{\sigma}(\eta)\right) \delta(\xi+\eta)=\left(\partial_{\xi} \hat{\Psi}_{\sigma}(\xi, \eta)-\hat{\Lambda}_{\sigma}^{t}(\xi) \hat{\Psi}_{\sigma}(\xi, \eta)\right) \delta(\xi+\eta) .
\end{gathered}
$$

Using the definitions of $\hat{\Psi}$ and $\hat{\Lambda}$ in (3.30), the phase-space realization (3.5) of the strip currents and the identities in (3.14), we have been able to confirm (after considerable algebra) that the integrability condition is satisfied. The inhomogeneous terms in Eq. (3.31) are boundary terms associated to the interaction between a non-abelian charge at $\xi$ and a non-abelian image charge at $-\eta$. Note that, because of these terms, the closed-string WZW orbifold bracket $\hat{\Delta}_{W Z W}(\xi, \eta, t, \sigma)=0$ is not a solution of the $\{\hat{x}, \hat{x}\}$ PDEs.

We may now follow Ref. 23] to find the following integral representation for the solution of Eq. (3.31):

$$
\begin{aligned}
\hat{\Delta}_{\sigma}(\xi, \eta)= & \hat{U}_{\sigma}^{t}(\xi) \hat{\Delta}_{\sigma}(0,0) \hat{U}_{\sigma}(\eta) \\
& +\pi i \int_{0}^{\eta} d \eta^{\prime}\left(\hat{\Psi}_{\sigma}\left(\xi, \eta^{\prime}\right) \delta\left(\xi+\eta^{\prime}\right)+\hat{U}_{\sigma}^{t}(\xi) \hat{\Psi}_{\sigma}\left(0, \eta^{\prime}\right) \delta\left(\eta^{\prime}\right)\right) \hat{U}_{\sigma}^{-1}\left(\eta^{\prime}\right) \hat{U}_{\sigma}(\eta) \\
& +\pi i \hat{U}_{\sigma}^{t}(\xi) \int_{0}^{\xi} d \xi^{\prime} \hat{U}_{\sigma}^{-1 t}\left(\xi^{\prime}\right)\left(\hat{\Psi}_{\sigma}\left(\xi^{\prime}, \eta\right) \delta\left(\xi^{\prime}+\eta\right)+\hat{\Psi}_{\sigma}\left(\xi^{\prime}, 0\right) \delta\left(\xi^{\prime}\right) \hat{U}_{\sigma}(\eta)\right) \\
& \partial_{\xi} \hat{U}_{\sigma}(\xi)=\hat{U}_{\sigma}(\xi) \hat{\Lambda}_{\sigma}(\xi), \quad \partial_{\eta} \hat{U}_{\sigma}^{t}(\eta)=\hat{\Lambda}_{\sigma}^{t}(\eta) \hat{U}_{\sigma}^{t}(\eta) \\
& \hat{U}_{\sigma}(0)=\hat{U}_{\sigma}^{t}(0)=\mathbb{1}, \quad \hat{U}_{\sigma}(\xi) \equiv \hat{U}(\xi, t, \sigma), \quad \hat{U}_{\sigma}^{t}(\eta) \equiv \hat{U}^{t}(\eta, t, \sigma) .
\end{aligned}
$$


In this solution, we have introduced the ordered product $\hat{U}_{\sigma}$ in each sector $\sigma$, and only the quantity $\hat{\Delta}_{\sigma}(0,0)=\hat{\Delta}(0,0, t, \sigma)$ is undetermined. With the integrability condition (3.32), it is straightforward to check directly that Eq. (3.33) solves Eq. (3.31). Moreover, the required antisymmetry of the coordinate bracket

$$
\hat{\Delta}_{\sigma}^{t}(\eta, \xi)=-\hat{\Delta}_{\sigma}(\xi, \eta)
$$

is guaranteed by the corresponding antisymmetric choice $\hat{\Delta}_{\sigma}^{t}(0,0)=-\hat{\Delta}_{\sigma}(0,0)$.

We may also evaluate the solution (3.33) explicitly, using the following integral identity

$$
\int_{0}^{\eta} d \eta^{\prime} f\left(\xi, \eta^{\prime}\right) \delta\left(\xi+\eta^{\prime}\right)= \begin{cases}\frac{1}{2} f(0,0) & \text { if } \xi=0, \eta \neq 0 \\ \frac{1}{2} f(\pi, \pi) & \text { if } \xi=\eta=\pi \\ 0 & \text { otherwise }\end{cases}
$$

to obtain the result:

$$
\hat{\Delta}_{\sigma}(\xi, \eta)= \begin{cases}\hat{\Delta}_{\sigma}(0,0) & \text { if } \xi=\eta=0 \\ \hat{U}_{\sigma}^{t}(\pi)\left(\hat{\Delta}_{\sigma}(0,0)+i \pi \hat{\Psi}_{\sigma}(0,0)\right) \hat{U}_{\sigma}(\pi)+i \pi \hat{\Psi}_{\sigma}(\pi, \pi) & \text { if } \xi=\eta=\pi \\ \hat{U}_{\sigma}^{t}(\xi)\left(\hat{\Delta}_{\sigma}(0,0)+i \pi \hat{\Psi}_{\sigma}(0,0)\right) \hat{U}_{\sigma}(\eta) & \text { otherwise. }\end{cases}
$$

As in Ref. [23], this expression for the coordinate bracket is suitable for the computation of $\xi$-derivatives in the bulk, but one must return to the form given in Eq. (3.33) in order to compute $\xi$-derivatives at the boundary.

The preferred choice for $\hat{\Delta}_{\sigma}(0,0)$ is therefore

$$
\hat{\Delta}_{\sigma}(0,0)=-i \pi \hat{\Psi}_{\sigma}(0,0)
$$

because in this case the twisted equal-time coordinate brackets vanish in the bulk, and are therefore locally twisted WZW:

$$
\left\{\hat{x}_{\sigma}^{n(r) \mu}(\xi), \hat{x}_{\sigma}^{n(s) \nu}(\eta)\right\}=\hat{\Delta}_{\sigma}^{n(r) \mu ; n(s) \nu}(\xi, \eta)= \begin{cases}-i \pi \hat{\Psi}_{\sigma}^{n(r) \mu ; n(s) \nu}(0,0) & \text { if } \xi=\eta=0 \\ i \pi \hat{\Psi}_{\sigma}^{n(r) \mu ; n(s) \nu}(\pi, \pi) & \text { if } \xi=\eta=\pi \\ 0 & \text { otherwise }\end{cases}
$$




$$
\begin{aligned}
& \hat{\Psi}_{\sigma}^{n(r) \mu ; n(s) \nu}(\xi, \xi)=-\hat{\Psi}_{\sigma}^{n(s) \nu ; n(r) \mu}(\xi, \xi) \\
& =\mathcal{G}^{n(t) \delta ; n(u) \epsilon}(\sigma) e^{-2 i \frac{n(t)}{\rho(\sigma)} \xi}\left(\hat{\bar{e}}^{-1}(\xi)_{n(t) \delta}^{n(r) \mu} \hat{e}^{-1}(\xi)_{n(u) \epsilon}^{n(s) \nu}-\hat{e}^{-1}(\xi)_{n(u) \epsilon}{ }^{n(r) \mu} \hat{\bar{e}}^{-1}(\xi)_{n(t) \delta}{ }_{n}^{n(s) \nu}\right) \\
& =\hat{e}^{-1}(\xi)_{n(u) \epsilon^{n(r) \mu}} \mathcal{G}^{n(u) \epsilon ; n(t) \delta}(\sigma) \times \\
& \times\left(e^{-2 i \frac{n(t)}{\rho(\sigma)} \xi} \hat{\Omega}^{-1}(\xi)_{n(t) \delta}^{n(v) \kappa}-\hat{\Omega}(\xi)_{n(t) \delta}^{n(v) \kappa} e^{\left.2 i \frac{n(v)}{\rho(\sigma) \xi}\right)} \hat{e}^{-1}(\xi)_{n(v) \kappa}^{n(s) \nu}\right. \\
& \sigma=0, \ldots, N_{c}-1 .
\end{aligned}
$$

These twisted coordinate brackets, which describe the new twisted non-commutative geometry of the open-string WZW orbifold $A_{g}^{\text {open }}(H) / H$, are a central result of this paper.

In the untwisted sector $(\sigma=0)$ of $A_{g}^{\text {open }}(H) / H$, the twisted coordinate brackets reduce to the following

$$
\begin{gathered}
\mathcal{G}(\sigma=0)=G, \quad \hat{e}(\xi, \sigma=0)=e(\xi), \quad \hat{\Psi}_{0}(\xi, \eta)=\Psi(\xi, \eta), \quad \hat{\Delta}_{0}(\xi, \eta)=\Delta(\xi, \eta) \\
\left\{x^{i}(\xi), x^{j}(\eta)\right\}=\Delta^{i j}(\xi, \eta)= \begin{cases}-i \pi \Psi^{i j}(0,0) & \text { if } \xi=\eta=0, \\
i \pi \Psi^{i j}(\pi, \pi) & \text { if } \xi=\eta=\pi, \\
0 & \text { otherwise, }\end{cases} \\
\Psi^{i j}(\xi, \xi)=\bar{e}(\xi)_{a}{ }^{i} G^{a b} e(\xi){ }_{b}{ }^{j}-e(\xi)_{a}{ }^{i} G^{a b} \bar{e}(\xi)_{b}{ }^{j} \\
=e(\xi){ }_{a}{ }^{i} G^{a c}\left(\Omega(\xi)^{-1}-\Omega(\xi){ }_{c}{ }^{b} e(\xi)_{b}{ }^{j}\right.
\end{gathered}
$$

which is recognized as the untwisted non-commutative geometry of Ref. [23, now for the special case of the untwisted open WZW string $A_{g}^{\text {open }}(H)$ with a symmetry $H$.

Using Eqs. (3.6), (3.38) and the chain rule, we have also computed the equal-time brackets of the group orbifold elements among themselves

$$
\begin{aligned}
& \left\{\hat{g}(\mathcal{T}, \xi)_{N(r) \mu}{ }^{N(s) \nu}, \hat{g}\left(\mathcal{T}^{\prime}, \eta\right)_{N^{\prime}\left(r^{\prime}\right) \mu^{\prime}}{ }^{N^{\prime}\left(s^{\prime}\right) \nu^{\prime}}\right\}= \\
& \begin{cases}i \pi\left(\hat{g}(\mathcal{T}, 0) \mathcal{T}_{n(t) \delta}\right)_{N(r) \mu}{ }^{N(s) \nu}\left(\hat{g}\left(\mathcal{T}^{\prime}, 0\right) \mathcal{T}_{n(u) \epsilon}^{\prime}\right)_{N^{\prime}\left(r^{\prime}\right) \mu^{\prime}}^{N^{\prime}\left(s^{\prime}\right) \nu^{\prime}} \times & \\
\mathcal{G}^{n(t) \delta ; n(v) \kappa}(\sigma)\left(\hat{\Omega}^{-1}(0)-\hat{\Omega}(0)\right)_{n(v) \kappa}{ }^{n(u) \epsilon} & \text { if } \xi=\eta=0, \\
-i \pi\left(\hat{g}(\mathcal{T}, \pi) \mathcal{T}_{n(t) \delta}\right)_{N(r) \mu}{ }^{N(s) \nu}\left(\hat{g}\left(\mathcal{T}^{\prime}, \pi\right) \mathcal{T}_{n(u) \epsilon}^{\prime}\right)_{N^{\prime}\left(r^{\prime}\right) \mu^{\prime}}^{N^{\prime}\left(s^{\prime}\right) \nu^{\prime} \times} & \\
\quad \mathcal{G}^{n(t) \delta ; n(v) \kappa}(\sigma)\left(e^{-2 \pi i \frac{n(v)}{\rho(\sigma)}} \hat{\Omega}^{-1}(\pi)_{n(v) \kappa}^{n(u) \epsilon}-\hat{\Omega}(\pi)_{n(v) \kappa}{ }^{n(u) \epsilon} e^{2 \pi i \frac{n(u)}{\rho(\sigma)}}\right) & \text { if } \xi=\eta=\pi, \\
0 & \text { otherwise }\end{cases} \\
& \hat{g}(\mathcal{T}, \xi) \equiv \hat{g}(\mathcal{T}, \xi, t, \sigma)
\end{aligned}
$$


where $\hat{\Omega}$ is the adjoint action defined in Eq. (3.14).

All other phase-space brackets

$$
\begin{gathered}
\left\{\hat{x}_{\sigma}^{n(r) \mu}(\xi, t), \hat{p}_{n(s) \nu}(\hat{B}, \eta, t)\right\}, \quad\left\{\hat{x}_{\sigma}^{n(r) \mu}(\xi, t), \hat{p}_{n(s) \nu}^{\sigma}(\eta, t)\right\}, \quad\left\{\hat{J}_{n(r) \mu}^{( \pm)}(\xi, t), \hat{p}_{n(s) \nu}(\hat{B}, \eta, t)\right\} \\
\left\{\hat{p}_{n(r) \mu}(\hat{B}, \xi, t), \hat{p}_{n(s) \nu}(\hat{B}, \eta, t)\right\}, \quad\left\{\hat{p}_{n(r) \mu}^{\sigma}(\xi, t), \hat{p}_{n(s) \nu}^{\sigma}(\eta, t)\right\}
\end{gathered}
$$

can now be straightforwardly computed (in the order listed) from the inverse relations (3.9) and the known brackets

$$
\left\{\hat{J}_{n(r) \mu}^{( \pm)}(\xi), \hat{J}_{n(s) \nu}^{( \pm)}(\eta)\right\}, \quad\left\{\hat{J}_{n(r) \mu}^{( \pm)}(\xi), \hat{x}_{\sigma}^{n(s) \nu}(\eta)\right\}, \quad\left\{\hat{x}_{\sigma}^{n(r) \mu}(\xi), \hat{x}_{\sigma}^{n(s) \nu}(\eta)\right\}
$$

without solving any additional differential equations. The corresponding results are given for the untwisted case in Ref. [23] (our sector $\sigma=0$ ), but owing to the length of these expressions, we will not reproduce the twisted analogues here.

Some explicit examples of the new twisted non-commutative geometry are given in App. D, and the following subsection works out the relatively simple quasi-canonical algebra of $\hat{x}_{\sigma}, \hat{p}^{\sigma}$ for a large class of open-string free-bosonic orbifolds.

\subsection{Example: Twisted Free-Bosonic Open Strings}

Applications of the orbifold program to closed-string free-bosonic orbifolds (including their twisted vertex operators and classical formulations) can be found in Refs. 7, 9, 11. In the following paragraph, we briefly review the classical development of closed-string freebosonic orbifolds.

One begins with the general untwisted left-mover free-boson sector on abelian $g$

$$
\begin{gathered}
f_{a b}^{c}=0, \quad e_{i}^{a}=-\bar{e}_{i}^{a}=\delta_{i}^{a}, \quad B_{i j}=H_{i j k}=0 \\
\left\{J_{a}(m), J_{b}(n)\right\}=m G_{a b} \delta_{m+n, 0}, \quad\left[T_{a}, T_{b}\right]=0
\end{gathered}
$$

which may be considered as a formal abelian limit ${ }^{\ddagger 5}$ of the untwisted WZW model $A_{g}(H)$. Under a general automorphism $\omega\left(h_{\sigma}\right) \in H \subset A u t(g)$, one finds the twisted current algebra of sector $\sigma$ of a closed-string free-bosonic orbifold [7, 11]. This gives us in particular the following left-mover input data for sector $\sigma$ of the corresponding open-string free-bosonic

\footnotetext{
${ }^{5}$ The automorphism group of the abelian current algebra is of course larger than the automorphism group of the non-abelian starting point; for example, $\omega\left(h_{\sigma}\right)=-\mathbb{1}$ appears in the abelian case.
} 
orbifold

$$
\begin{gathered}
\mathcal{F}_{n(r) \mu ; n(s) \nu}{ }^{n(t) \delta}(\sigma)=0, \quad\left[\mathcal{T}_{n(r) \mu}, \mathcal{T}_{n(s) \nu}\right]=0 \\
\left\{\hat{J}_{n(r) \mu}\left(m+\frac{n(r)}{\rho(\sigma)}\right), \hat{J}_{n(s) \nu}\left(n+\frac{n(s)}{\rho(\sigma)}\right)\right\}=\left(m+\frac{n(r)}{\rho(\sigma)}\right) \delta_{m+n+\frac{n(r)+n(s)}{\rho(\sigma)}, 0} \mathcal{G}_{n(r) \mu ;-n(r), \nu}(\sigma) \\
\left\{\hat{J}_{n(r) \mu}\left(m+\frac{n(r)}{\rho(\sigma)}\right), \hat{g}(\mathcal{T}, \xi, t)\right\}=\hat{g}(\mathcal{T}, \xi, t) \mathcal{T}_{n(r) \mu} e^{i\left(m+\frac{n(r)}{\rho(\sigma)}\right)(t+\xi)} \\
-\mathcal{T}_{n(r) \mu} \hat{g}(\mathcal{T}, \xi, t) e^{i\left(m+\frac{n(r)}{\rho(\sigma)}\right)(t-\xi)} \\
\hat{e}_{n(r) \mu}{ }^{n(s) \nu}=-\hat{\bar{e}}_{n(r) \mu}{ }^{n(s) \nu}=\delta_{\mu}^{\nu} \delta_{n(r)-n(s), 0 \bmod \rho(\sigma)} \\
\hat{G}_{n(r) \mu ; n(s) \nu}=\mathcal{G}_{n(r) \mu ; n(s) \nu}(\sigma) \\
\hat{B}_{n(r) \mu ; n(s) \nu}=\hat{H}_{n(r) \mu ; n(s) \nu ; n(t) \delta}=0, \quad \sigma=0, \ldots, N_{c}-1
\end{gathered}
$$

which also may be considered as a formal abelian limit of the left-mover WZW orbifold data. In these results, Eq. (3.44e) records that, for free bosons, the twisted Einstein metric $\hat{G}$ is equal [11] to the twisted tangent space metric $\mathcal{G}$ (see Eqs. (2.2a) and (3.7c)). As is clear from Eqs. (3.43a and (3.44f), we are not treating the most general twisted freebosonic open string in the basic class, which can also involve $\hat{B} \neq 0$. Similarly, we will not discuss any particular target-space compactification of the twisted open-string coordinates $\hat{x}_{\sigma}$ below.

Further classical description of these twisted free-bosonic open strings is obtained by substituting the data (3.44) into the more general WZW results above. For example, the phase-space realization (3.5) of the twisted open string reduces to the following:

$$
\begin{gathered}
\hat{J}_{n(r) \mu}^{(+)}(\xi, t)=2 \pi \hat{p}_{n(r) \mu}^{\sigma}(\xi, t)+\frac{1}{2} \partial_{\xi} \hat{x}_{\sigma}^{n(s) \nu}(\xi, t) \mathcal{G}_{n(s) \nu ; n(r) \mu}(\sigma) \\
\hat{J}_{n(r) \mu}^{(-)}(\xi, t)=-2 \pi \hat{p}_{n(r) \mu}^{\sigma}(\xi, t)+\frac{1}{2} \partial_{\xi} \hat{x}_{\sigma}^{n(s) \nu}(\xi, t) \mathcal{G}_{n(s) \nu ; n(r) \mu}(\sigma) \\
\hat{J}_{n(r) \mu}^{( \pm)}(\xi, t)=\sum_{m \in \mathbb{Z}} \hat{J}_{n(r) \mu}\left(m+\frac{n(r)}{\rho(\sigma)}\right) e^{-i\left(m+\frac{n(r)}{\rho(\sigma)}\right)(t \pm \xi)} .
\end{gathered}
$$

As another example, the boundary conditions in Eq. (3.23) now take the simple form

$$
\begin{gathered}
\partial_{t} \hat{x}_{\sigma}^{n(r) \mu}(\xi, t)= \begin{cases}0 & \text { at } \xi=0 \\
i \tan \left(\frac{\pi n(r)}{\rho(\sigma)}\right) \partial_{\xi} \hat{x}_{\sigma}^{n(r) \mu}(\xi, t) & \text { at } \xi=\pi\end{cases} \\
\{\bar{n}(r)\} \subset\{0, \ldots, \rho(\sigma)-1\}, \quad \sigma=0, \ldots, N_{c}-1
\end{gathered}
$$

which describe the branes at each end of the twisted free-bosonic open string. Each sector $\sigma$ of the open-string free-bosonic orbifold comes equipped (via the $H$-eigenvalue problem 
(2.3a) ) with a specific set $\{n(r)\}$ of spectral indices and hence a corresponding specific set of boundary conditions (3.46) on the coordinates $\hat{x}_{\sigma}^{n(r) \mu}$.

In the untwisted sector $(\sigma=\bar{n}(r)=0)$ we have $n(r) \mu \rightarrow i$ and these abelian results reduce to Dirichlet-Dirichlet (D-D) strings (with $\partial_{t} x^{i}=0$ at both ends), as discussed in Ref. [23]. More generally, all the twisted coordinates are Dirichlet at $\xi=0$ in every sector $\sigma$. One also finds that the coordinates $\hat{x}_{\sigma}^{0 \mu}(\xi, t)$ always have D-D boundary conditions, and furthermore coordinates $\hat{x}_{\sigma}^{n(r) \mu}(\xi, t)$ with $\frac{\bar{n}(r)}{\rho(\sigma)}=\frac{1}{2}$ have Dirichlet-Neumann (D-N) boundary conditions (with $\partial_{\xi} \hat{x}_{\sigma}=0$ at $\xi=\pi$ ). As an illustration, the simple $\mathbb{Z}_{2}$ permutation symmetry $x^{1} \leftrightarrow x^{2}$ with $G_{a b}=\delta_{a b}$ leads to the abelian orbifold affine algebra

$$
\begin{gathered}
\left\{\hat{J}_{n(r)}\left(m+\frac{n(r)}{2}\right), \hat{J}_{n(s)}\left(n+\frac{n(s)}{2}\right)\right\}=\left(m+\frac{n(r)}{2}\right) 2 \delta_{m+n+\frac{n(r)+n(s)}{2}, 0} \\
\rho(\sigma)=2, \quad \bar{n}(r), \bar{n}(s) \in\{0,1\}
\end{gathered}
$$

and the corresponding open-string coordinates $\hat{x}^{n(r)}$ of this twisted sector have boundary conditions D-D for $\bar{n}(r)=0$ and D-N for $\bar{n}(r)=1$. However, in more general examples (with other values of $\frac{\bar{n}(r)}{\rho(\sigma)}$ ), the sets of boundary conditions in each sector are larger and have more complicated (mixed) behavior at $\xi=\pi$, as seen in Eq. (3.46). Mixed boundary conditions with $\hat{B}=0$ have also been observed in the open-string sectors of free-bosonic orientation orbifolds [17.

In the remainder of this subsection, we will obtain the full quasi-canonical algebra of these twisted free-bosonic open strings, including in particular the non-commutative geometry of the $\{\hat{x}, \hat{x}\}$ brackets.

The non-commutative geometry of sector $\sigma$ of the open-string free-bosonic orbifold follows immediately in the abelian limit of the $\{\hat{x}, \hat{x}\}$ brackets (3.38)

$$
\begin{aligned}
& \hat{\Lambda}_{\sigma}(\xi, t)=0, \quad \hat{U}_{\sigma}(\xi, t)=1 \\
& \hat{\Psi}^{n(r) \mu ; n(s) \nu}(\xi, \xi, t, \sigma)=2 i \mathcal{G}^{n(r) \mu ; n(s) \nu}(\sigma) \sin \left(2 \frac{n(r)}{\rho(\sigma)} \xi\right), \quad \sigma=0, \ldots, N_{c}-1 \\
& \left\{\hat{x}_{\sigma}^{n(r) \mu}(\xi, t), \hat{x}_{\sigma}^{n(s) \nu}(\eta, t)\right\}= \\
& -2 \pi \delta_{n(r)+n(s), 0 \bmod \rho(\sigma)} \mathcal{G}^{n(r) \mu ;-n(r), \nu}(\sigma) \begin{cases}0 & \text { if } \xi=\eta=0, \\
\sin \left(\frac{2 \pi n(r)}{\rho(\sigma)}\right) & \text { if } \xi=\eta=\pi, \\
0 & \text { otherwise }\end{cases}
\end{aligned}
$$

where $\mathcal{G}^{\bullet}(\sigma)$ is the inverse of the twisted tangent space metric in Eq. (2.2). We remark in particular that this geometry becomes commutative when $\frac{\bar{n}(r)}{\rho(\sigma)}=0$ or $\frac{1}{2}$ (i. e. D-D or D-N coordinates), which includes the untwisted sector $\sigma=0$ (see Ref. [23]). 
To find the other brackets among $\hat{x}_{\sigma}$ and $\hat{p}^{\sigma}$, we begin with the equations of motion

$$
\begin{aligned}
& \partial_{t} \hat{x}_{\sigma}^{n(r) \mu}(\xi, t)=\mathcal{G}^{n(r) \mu ; n(s) \nu}(\sigma)\left(\hat{J}_{n(s) \nu}^{(+)}(\xi, t)-\hat{J}_{n(s) \nu}^{(-)}(\xi, t)\right) \\
& \partial_{\xi} \hat{x}_{\sigma}^{n(r) \mu}(\xi, t)=\mathcal{G}^{n(r) \mu ; n(s) \nu}(\sigma)\left(\hat{J}_{n(s) \nu}^{(+)}(\xi, t)+\hat{J}_{n(s) \nu}^{(-)}(\xi, t)\right)
\end{aligned}
$$

which are the abelian limits of Eqs. (3.9a) and (3.18a ). Note that the boundary conditions in Eq. (3.46) also follow with the mode expansions (3.45c) from these equations of motion.

The solution of the equations of motion for the twisted coordinates is:

$$
\begin{gathered}
\hat{x}_{\sigma}^{0 \mu}(\xi, t)=\hat{q}^{0 \mu}+2 \mathcal{G}^{0 \mu ; 0 \nu}(\sigma)\left(\hat{J}_{0 \nu}(0) \xi+\sum_{m \neq 0} \frac{\hat{J}_{0 \nu}(m)}{m} e^{-i m t} \sin (m \xi)\right) \\
\bar{n}(r) \neq 0: \hat{x}_{\sigma}^{n(r) \mu}(\xi, t)=\hat{q}^{n(r) \mu}+ \\
+2 \mathcal{G}^{n(r) \mu ; n(s) \nu}(\sigma) \sum_{m \in \mathbb{Z}} \frac{\hat{J}_{n(s) \nu}\left(m+\frac{n(s)}{\rho(\sigma)}\right)}{m+\frac{n(s)}{\rho(\sigma)}} e^{-i\left(m+\frac{n(s)}{\rho(\sigma)}\right) t} \sin \left(m+\frac{n(s)}{\rho(\sigma)}\right) \xi .
\end{gathered}
$$

At this point, it is helpful to note the following algebraic requirement

$$
\left\{\hat{J}_{n(r) \mu}\left(m+\frac{n(r)}{\rho(\sigma)}\right), \hat{x}^{n(s) \nu}(\xi, t)\right\}=2 \delta_{n(r) \mu}{ }^{n(s) \nu} e^{i\left(m+\frac{n(r)}{\rho(\sigma)}\right) t} \sin \left(m+\frac{n(r)}{\rho(\sigma)}\right) \xi
$$

which follows from Eq. (3.45c) and the abelian limit of Eq. (3.12). We find that the mode expansions in Eq. (3.50) are consistent with Eq. (3.51) only when we choose:

$$
\left\{\hat{J}_{n(r) \mu}\left(m+\frac{n(r)}{\rho(\sigma)}\right), \hat{q}^{n(s) \nu}\right\}=0 .
$$

Jacobi identities then tell us that the bracket of any two $\hat{q}$ 's is at most a constant, which we will tentatively assume to be zero

$$
\left\{\hat{q}^{n(r) \mu}, \hat{q}^{n(s) \nu}\right\}=0
$$

so that the $\hat{q}$ 's are simply c-numbers.

We will also need the free-bosonic momenta

$$
\hat{p}_{n(r) \mu}^{\sigma}(\xi, t)=\frac{1}{4 \pi}\left(\hat{J}_{n(r) \mu}^{(+)}(\xi, t)-\hat{J}_{n(r) \mu}^{(-)}(\xi, t)\right)
$$

which are obtained in the abelian limit of Eq. (3.9b). With these momenta, the mode expansions (3.45c), (3.50) and the current algebra (3.44b), we may now compute the full quasi-canonical algebra of $\hat{x}_{\sigma}, \hat{p}^{\sigma}$

$$
\begin{gathered}
\left\{\hat{p}_{n(r) \mu}^{\sigma}(\xi, t), \hat{p}_{n(s) \nu}^{\sigma}(\eta, t)\right\}=0 \\
\left\{\hat{x}_{\sigma}^{n(r) \mu}(\xi, t), \hat{p}_{n(s) \nu}^{\sigma}(\eta, t)\right\}=i \delta_{n(s) \nu}^{n(r) \mu}\left(\delta(\xi-\eta)-\cos \left(\frac{n(s)}{\rho(\sigma)}(\xi+\eta)\right) \delta(\xi+\eta)\right)
\end{gathered}
$$




$$
\begin{array}{r}
\left\{\hat{x}_{\sigma}^{n(r) \mu}(\xi, t), \hat{x}_{\sigma}^{n(s) \nu}(\eta, t)\right\}= \\
-2 \pi \delta_{n(r)+n(s), 0 \bmod \rho(\sigma)} \mathcal{G}^{n(r) \mu ;-n(r), \nu}(\sigma) \begin{cases}0 & \text { if } \xi=\eta=0, \\
\sin \left(\frac{2 \pi n(r)}{\rho(\sigma)}\right) & \text { if } \xi=\eta=\pi, \\
0 & \text { otherwise }\end{cases} \\
\sigma=0, \ldots, N_{c}-1
\end{array}
$$

in each sector of each open-string free-bosonic orbifold. To obtain these results, we found that the following identities citeOrient1 were useful:

$$
\begin{aligned}
& \sin \left(\frac{n(r)}{\rho(\sigma)}(\xi-\eta)\right) \delta(\xi-\eta)=0, \quad 0 \leq \xi, \eta \leq \pi \\
& \cos \left(\frac{n(r)}{\rho(\sigma)}(\xi-\eta)\right) \delta(\xi-\eta)=\delta(\xi-\eta), \quad 0 \leq \xi, \eta \leq \pi \\
& \sin \left(\frac{n(r)}{\rho(\sigma)}(\xi+\eta)\right) \delta(\xi+\eta)=0 \text { except at } \xi=\eta=\pi \\
& \sum_{m \in \mathbb{Z}} \frac{1}{m+\frac{n(r)}{\rho(\sigma)}} \sin \left(\left(m+\frac{n(r)}{\rho(\sigma)}\right) \xi\right) \sin \left(\left(m+\frac{n(r)}{\rho(\sigma)}\right) \eta\right)= \\
& m+\frac{n(r)}{\rho(\sigma)} \neq 0 \\
& =\pi \int_{\xi-\eta}^{\xi+\eta} d \eta^{\prime} \sin \left(\frac{n(r)}{\rho(\sigma)} \eta^{\prime}\right) \delta\left(\eta^{\prime}\right)=\left\{\begin{array}{cl}
0 & \text { if } \xi=\eta=0 \\
\frac{\pi}{2} \sin \left(\frac{2 \pi n(r)}{\rho(\sigma)}\right) & \text { if } \xi=\eta=\pi \\
0 & \text { otherwise }
\end{array}\right.
\end{aligned}
$$

Following standard procedure in distribution theory, the sum in Eq. (3.56d) was defined by temporarily inserting a smearing function $\exp \left(-\epsilon m^{2}\right), \epsilon \rightarrow 0^{+}$.

We call the algebras in Eq. (3.55) quasi-canonical because they are canonical in the bulk, and we also note that the result for the $\{\hat{x}, \hat{x}\}$ bracket in Eq. (3.55c) is in agreement with Eq. (3.48c). Allowing constant non-zero $\{\hat{q}, \hat{q}\}$ brackets gives rise to overall additive constants in the $\{\hat{x}, \hat{x}\}$ brackets, so the assumption in Eq. (3.53) is equivalent to our earlier requirement that $\{\hat{x}, \hat{x}\}$ vanishes in the bulk.

\section{Conformal Field Theory of Twisted Open WZW Strings}

In this section, we further develop (see Sec. 2) the quantum theory of our basic class of twisted open WZW strings. This discussion culminates in the derivation of the twisted open-string KZ equations in Subsec. 4.6. 


\subsection{The Quantum Hamiltonian and the Twisted Affine Primary Fields}

Combining Eqs. (A.3a) and (2.15), we obtain the quantum Hamiltonian of sector $\sigma$ of $A_{g}^{\text {open }}(H) / H$ :

$$
\begin{aligned}
& \hat{H}_{\sigma}=L_{\sigma}(0)= \\
& =\frac{1}{2 \pi} \int_{0}^{\pi} d \xi \mathcal{L}_{\hat{\mathfrak{g}}(\sigma)}^{n(r) \mu ;-n(r), \nu}(\sigma)\left(: \hat{J}_{n(r) \mu}^{(+)}(\xi, t) \hat{J}_{-n(r), \nu}^{(+)}(\xi, t)+\hat{J}_{n(r) \mu}^{(-)}(\xi, t) \hat{J}_{-n(r), \nu}^{(-)}(\xi, t):\right) \\
& =\mathcal{L}_{\hat{\mathfrak{g}}(\sigma)}^{n(r) \mu ;-n(r), \nu}(\sigma)\left(\sum_{p \in \mathbb{Z}}: \hat{J}_{n(r) \mu}\left(p+\frac{n(r)}{\rho(\sigma)}\right) \hat{J}_{-n(r), \nu}\left(-p-\frac{n(r)}{\rho(\sigma)}\right):_{M}\right. \\
& \left.-i \frac{\bar{n}(r)}{\rho(\sigma)} \mathcal{F}_{n(r) \mu ;-n(r), \nu}^{0, \delta}(\sigma) \hat{J}_{0, \delta}(0)\right)+\hat{\Delta}_{0}(\sigma) \\
& : \hat{J}_{n(r) \mu}\left(m+\frac{n(r)}{\rho(\sigma)}\right) \hat{J}_{n(s) \nu}\left(n+\frac{n(s)}{\rho(\sigma)}\right):_{M} \equiv \theta\left(m+\frac{n(r)}{\rho(\sigma)} \geq 0\right) \hat{J}_{n(s) \nu}\left(n+\frac{n(s)}{\rho(\sigma)}\right) \hat{J}_{n(r) \mu}\left(m+\frac{n(r)}{\rho(\sigma)}\right) \\
& +\theta\left(m+\frac{n(r)}{\rho(\sigma)}<0\right) \hat{J}_{n(r) \mu}\left(m+\frac{n(r)}{\rho(\sigma)}\right) \hat{J}_{n(s) \nu}\left(n+\frac{n(s)}{\rho(\sigma)}\right) \\
& {\left[\hat{J}_{n(r) \mu}\left(m+\frac{n(r)}{\rho(\sigma)}\right), \hat{J}_{n(s) \nu}\left(n+\frac{n(s)}{\rho(\sigma)}\right)\right]=i \mathcal{F}_{n(r) \mu ; n(s) \nu} \frac{n(r)+n(s), \delta}{(\sigma)} \hat{J}_{n(r)+n(s), \delta}\left(m+n+\frac{n(r)+n(s)}{\rho(\sigma)}\right)} \\
& +\left(m+\frac{n(r)}{\rho(\sigma)}\right) \delta_{m+n+\frac{n(r)+n(s)}{\rho(\sigma)}, 0} \mathcal{G}_{n(r) \mu ;-n(r) \nu}(\sigma) \\
& \partial_{t} \hat{A}(\xi, t)=i\left[\hat{H}_{\sigma}, \hat{A}(\xi, t)\right] .
\end{aligned}
$$

Here we have also collected Eqs. (A.3c), (A.1) and (2.16a) for reference. Explicit formulae for the conformal weight $\hat{\Delta}_{0}(\sigma)$ of the scalar twist-field state are given in Eqs. (A.3e) and (2.22d).

Using the classical theory and in particular the $\{\hat{J}, \hat{g}\}$ brackets in Eq. (3.16) as a guide, we may augment the quantum theory with the following equal-time commutators

$$
\begin{array}{r}
{\left[\hat{J}_{n(r) \mu}^{(+)}(\xi, t), \hat{g}(\mathcal{T}, \eta, t)\right]=2 \pi\left(\hat{g}(\mathcal{T}, \eta, t) \mathcal{T}_{n(r) \mu} \delta_{\frac{n(r)}{\rho(\sigma)}}(\xi-\eta)\right.} \\
\left.-\mathcal{T}_{n(r) \mu} \hat{g}(\mathcal{T}, \eta, t) \delta_{\frac{n(r)}{\rho(\sigma)}}(\xi+\eta)\right) \\
{\left[\hat{J}_{n(r) \mu}^{(-)}(\xi, t), \hat{g}(\mathcal{T}, \eta, t)\right]=2 \pi\left(-\mathcal{T}_{n(r) \mu} \hat{g}(\mathcal{T}, \eta, t) \delta_{-\frac{n(r)}{\rho(\sigma)}}(\xi-\eta)\right.} \\
\left.+\hat{g}(\mathcal{T}, \eta, t) \mathcal{T}_{n(r) \mu} \delta_{-\frac{n(r)}{\rho(\sigma)}}(\xi+\eta)\right)
\end{array}
$$


where this $\hat{g}(\mathcal{T})$ is the open-string twisted affine primary field in twisted rep $\mathcal{T}$. The (classical) group orbifold element $\hat{g}(\mathcal{T})$ of Sec. 3 is the high-level limit of this operator field. As noted above for the corresponding brackets in Eq. (3.16), the commutators (4.2) satisfy the $\hat{J}, \hat{J}, \hat{g}$ operator Jacobi identities.

Moreover, using the mode expansions (2.5) of the twisted strip currents, we find that the mode commutator

$$
\begin{array}{r}
{\left[\hat{J}_{n(r) \mu}\left(m+\frac{n(r)}{\rho(\sigma)}\right), \hat{g}(\mathcal{T}, \xi, t)\right]=\hat{g}(\mathcal{T}, \xi, t) \mathcal{T}_{n(r) \mu} e^{i\left(m+\frac{n(r)}{\rho(\sigma)}\right)(t+\xi)}} \\
\quad-\mathcal{T}_{n(r) \mu} \hat{g}(\mathcal{T}, \xi, t) e^{i\left(m+\frac{n(r)}{\rho(\sigma)}\right)(t-\xi)}
\end{array}
$$

is equivalent to both Eqs. (4.2a) and (4.2b).

\subsection{Time Dependence of the Twisted Affine Primary Fields}

Using Eqs. (4.1) and (4.2), we find after some algebra the time evolution of the open-string twisted affine primary fields

$$
\begin{aligned}
\partial_{t} \hat{g}(\mathcal{T}, \xi, t)=i\left[\hat{H}_{\sigma}, \hat{g}(\mathcal{T}, \xi, t)\right] \\
=2 i \mathcal{L}_{\hat{\mathfrak{g}}(\sigma)}^{n(r) \mu ;-n(r), \nu}(\sigma)\left(: \hat{J}_{n(r) \mu}^{(+)}(\xi, t) \hat{g}(\mathcal{T}, \xi, t) \mathcal{T}_{-n(r), \nu}-\mathcal{T}_{n(r) \mu} \hat{J}_{-n(r), \nu}^{(-)}(\xi, t) \hat{g}(\mathcal{T}, \xi, t):_{M}\right) \\
\quad+i \mathcal{L}_{\hat{\mathfrak{g}}(\sigma)}^{0, \mu ; 0, \nu}(\sigma)\left(\hat{g}(\mathcal{T}, \xi, t) \mathcal{T}_{0, \mu} \mathcal{T}_{0, \nu}+\mathcal{T}_{0, \mu} \mathcal{T}_{0, \nu} \hat{g}(\mathcal{T}, \xi, t)-2 \mathcal{T}_{0, \mu} \hat{g}(\mathcal{T}, \xi, t) \mathcal{T}_{0, \nu}\right) \\
\quad+\mathcal{L}_{\hat{\mathfrak{g}}(\sigma)}^{n(r) \mu ;-n(r), \nu}(\sigma) \frac{\bar{n}(r)}{\rho(\sigma)} \mathcal{F}_{n(r) \mu ;-n(r), \nu}^{0, \delta}(\sigma)\left(\hat{g}(\mathcal{T}, \xi, t) \mathcal{T}_{0, \delta}-\mathcal{T}_{0, \delta} \hat{g}(\mathcal{T}, \xi, t)\right)
\end{aligned}
$$

which provides our first example of a twisted vertex operator equation. The mode normal ordering : $\cdot:_{M}$ in Eq. (4.4) is defined as follows

$$
\begin{aligned}
: \hat{J}_{n(r) \mu}^{( \pm)}(\xi, t) \hat{g}(\mathcal{T}, \xi, t) & :_{M} \equiv \hat{J}_{n(r) \mu}^{( \pm)-}(\xi, t) \hat{g}(\mathcal{T}, \xi, t)+\hat{g}(\mathcal{T}, \xi, t) \hat{J}_{n(r) \mu}^{( \pm)+}(\xi, t) \\
\hat{J}_{n(r) \mu}^{( \pm)+}(\xi, t) & \equiv \sum_{m \geq 0} \hat{J}_{\bar{n}(r) \mu}\left(m+\frac{\bar{n}(r)}{\rho(\sigma)}\right) e^{-i\left(m+\frac{\bar{n}(r)}{\rho(\sigma)}\right)(t \pm \xi)} \\
\hat{J}_{n(r) \mu}^{( \pm)-}(\xi, t) & \equiv \sum_{m \leq-1} \hat{J}_{\bar{n}(r) \mu}\left(m+\frac{\bar{n}(r)}{\rho(\sigma)}\right) e^{-i\left(m+\frac{\bar{n}(r)}{\rho(\sigma)}\right)(t \pm \xi)} \\
\hat{J}_{n(r) \mu}^{( \pm)+}(\xi, t)+\hat{J}_{n(r) \mu}^{( \pm)-}(\xi, t) & =\hat{J}_{n(r) \mu}^{( \pm)}(\xi, t)
\end{aligned}
$$

where $\hat{J}^{( \pm) \pm}$are called the partial currents. Since $\mathcal{L} \rightarrow \mathcal{G}^{\bullet} / 2$ and $\hat{J}=\mathcal{O}\left(k^{\frac{1}{2}}\right)$ in the highlevel limit, the classical equation of motion in Eq. (3.19a) is indeed the classical limit of the twisted vertex operator equation (4.4). 
An alternate form of the open-string twisted vertex operator equation (4.4b) is the following

$$
\begin{aligned}
& \partial_{t} \hat{g}(\mathcal{T}, \xi, t)= \\
& 2 i \mathcal{L}_{\hat{\mathfrak{g}}(\sigma)}^{n(r) \mu ;-n(r), \nu}(\sigma)\left(: \hat{J}_{n(r) \mu}^{(+)}(\xi, t) \hat{g}(\mathcal{T}, \xi, t) \mathcal{T}_{-n(r), \nu}-\mathcal{T}_{n(r) \mu} \hat{J}_{-n(r), \nu}^{(-)}(\xi, t) \hat{g}(\mathcal{T}, \xi, t):_{M}\right) \\
& +i\left[\mathcal{D}_{\hat{\mathfrak{g}}(\sigma)}(\mathcal{T}), \hat{g}(\mathcal{T}, \xi, t)\right]_{+}-2 i \mathcal{L}_{\hat{\mathfrak{g}}(\sigma)}^{0, \mu ; 0, \nu}(\sigma) \mathcal{T}_{0, \mu} \hat{g}(\mathcal{T}, \xi, t) \mathcal{T}_{0, \nu} \\
& -2 i \mathcal{L}_{\hat{\mathfrak{g}}(\sigma)}^{n(r) \mu ;-n(r), \nu}(\sigma)\left(\frac{\bar{n}(r)}{\rho(\sigma)} \hat{g}(\mathcal{T}, \xi, t) \mathcal{T}_{n(r) \mu} \mathcal{T}_{-n(r), \nu}+\overline{\overline{-n(r)}} \mathcal{T}_{n(r) \mu} \mathcal{T}_{-n(r), \nu} \hat{g}(\mathcal{T}, \xi, t)\right) \\
& \mathcal{D}_{\hat{\mathfrak{g}}(\sigma)}(\mathcal{T}) \equiv \mathcal{L}_{\hat{\mathfrak{g}}(\sigma)}^{n(r) \mu ;-n(r), \nu}(\sigma) \mathcal{T}_{n(r) \mu} \mathcal{T}_{-n(r), \nu}=U(T, \sigma) D_{g}(T) U^{\dagger}(T, \sigma) \\
& D_{g}(T)=L_{g}^{a b} T_{a} T_{b}
\end{aligned}
$$

where $\overline{-n(r)}$, which is the pullback of $-n(r)$, is formally defined in Eq. (A.3f). The quantity $\mathcal{D}_{\hat{\mathfrak{g}}(\sigma)}(\mathcal{T})$ is called the twisted conformal weight matrix [6] and $D_{g}(T)$ is the untwisted conformal weight matrix of rep $T$ of $g$. To obtain this result, we used the identities:

$$
\begin{aligned}
& 2 \mathcal{L}_{\hat{\mathfrak{g}}(\sigma) \mu ;-n(r), \nu}^{n(r)} \frac{\bar{n}(r)}{\rho(\sigma)} \mathcal{T}_{n(r) \mu} \mathcal{T}_{-n(r), \nu}= \\
& \quad=\mathcal{L}_{\hat{\mathfrak{g}}(\sigma) \mu}^{n(r) \mu ;-n(r), \nu}(\sigma)\left(\mathcal{T}_{n(r) \mu} \mathcal{T}_{-n(r), \nu}\left(1-\delta_{\bar{n}(r), 0}\right)+i \frac{\bar{n}(r)}{\rho(\sigma)} \mathcal{F}_{n(r) \mu ;-n(r), \nu}{ }^{0, \delta}(\sigma) \mathcal{T}_{0, \delta}\right) \\
& 2 \mathcal{L}_{\hat{\mathfrak{g}}(\sigma)}^{n(r) \mu ;-n(r), \nu} \frac{\bar{n}(r)}{\rho(\sigma)} \mathcal{T}_{-n(r), \nu} \mathcal{T}_{n(r) \mu}= \\
& \quad=\mathcal{L}_{\hat{\mathfrak{g}}(\sigma) \mu ;-n(r), \nu}^{n(\sigma)}(\sigma)\left(\mathcal{T}_{n(r) \mu} \mathcal{T}_{-n(r), \nu}\left(1-\delta_{\bar{n}(r), 0}\right)-i \frac{\bar{n}(r)}{\rho(\sigma)} \mathcal{F}_{n(r) \mu ;-n(r), \nu}{ }^{0, \delta}(\sigma) \mathcal{T}_{0, \delta}\right) .
\end{aligned}
$$

The identity (4.7a) was given in Ref. [6], and Eq. (4.7b) follows easily from Eq. (4.7a) and the orbifold Lie algebra (2.2f) .

We consider next the correlators $\hat{A}_{\sigma}$ of the open-string twisted affine primary fields in the scalar twist-field state (see Eq. (2.21)). With Eq. (4.3), we immediately obtain the global Ward identities for these correlators

$$
\begin{gathered}
\hat{A}_{\sigma}(\mathcal{T}, \xi, t) \equiv\left\langle\hat{g}\left(\mathcal{T}^{(1)}, \xi_{1}, t_{1}\right) \ldots \hat{g}\left(\mathcal{T}^{(n)}, \xi_{n}, t_{n}\right)\right\rangle_{\sigma} \\
\equiv{ }_{\sigma}\left\langle 0\left|\hat{g}\left(\mathcal{T}^{(1)}, \xi_{1}, t_{1}\right) \ldots \hat{g}\left(\mathcal{T}^{(n)}, \xi_{n}, t_{n}\right)\right| 0\right\rangle_{\sigma} \\
\left\langle\left[\hat{J}_{0, \mu}(0), \hat{g}\left(\mathcal{T}^{(1)}, \xi_{1}, t_{1}\right) \ldots \hat{g}\left(\mathcal{T}^{(n)}, \xi_{n}, t_{n}\right)\right]\right\rangle_{\sigma}=0 \\
\Rightarrow \hat{A}_{\sigma}(\mathcal{T}, \xi, t) \sum_{i=1}^{n} \mathcal{T}_{0, \mu}^{(i)}-\sum_{i=1}^{n} \mathcal{T}_{0, \mu}^{(i)} \hat{A}_{\sigma}(\mathcal{T}, \xi, t)=0
\end{gathered}
$$

which generalize the untwisted open-string Ward identities of Ref. 23. 
Towards obtaining twisted open-string KZ equations for these correlators, we give the following properties of the scalar twist-field state

$$
\hat{J}_{n(r) \mu}^{( \pm)+}(\xi, t)|0\rangle_{\sigma}={ }_{\sigma}\langle 0| \hat{J}_{n(r) \mu}^{( \pm)-}(\xi, t)=0
$$

which follow immediately from Eq. (2.21) and the definition of the partial currents in Eqs. (4.5b,c). With these properties and the twisted vertex operator equation (4.4), we may obtain the time derivatives of the open-string correlators $\hat{A}_{\sigma}$ :

$$
\begin{aligned}
& \partial_{t_{i}} \hat{A}_{\sigma}=2 i \mathcal{L}_{\hat{\mathfrak{g}}(\sigma)}^{n(r) \mu ;-n(r), \nu}(\sigma) \times \\
& \times \sum_{j \neq i}\left\{\frac{e^{i \frac{\bar{n}(r)}{\rho(\sigma)}\left(\phi_{j}-\phi_{i}\right)}}{1-e^{i\left(\phi_{j}-\phi_{i}\right)}} \hat{A}_{\sigma} \mathcal{T}_{n(r) \mu}^{(j)} \mathcal{T}_{-n(r), \nu}^{(i)}-\frac{e^{i \frac{\bar{n}(r)}{\rho(\sigma)}\left(\bar{\phi}_{j}-\phi_{i}\right)}}{1-e^{i\left(\bar{\phi}_{j}-\phi_{i}\right)}} \mathcal{T}_{n(r) \mu}^{(j)} \hat{A}_{\sigma} \mathcal{T}_{-n(r), \nu}^{(i)}\right. \\
& \left.-\frac{e^{i \frac{\bar{n}(r)}{\rho(\sigma)}\left(\phi_{j}-\bar{\phi}_{i}\right)}}{1-e^{i\left(\phi_{j}-\bar{\phi}_{i}\right)}} \mathcal{T}_{-n(r), \nu}^{(i)} \hat{A}_{\sigma} \mathcal{T}_{n(r) \mu}^{(j)}+\frac{e^{i \frac{\bar{n}(r)}{\rho(\sigma)}\left(\bar{\phi}_{j}-\bar{\phi}_{i}\right)}}{1-e^{i\left(\bar{\phi}_{j}-\bar{\phi}_{i}\right)}} \mathcal{T}_{-n(r), \nu}^{(i)} \mathcal{T}_{n(r) \mu}^{(j)} \hat{A}_{\sigma}\right\} \\
& +i\left[\mathcal{D}_{\hat{\mathfrak{g}}(\sigma)}\left(\mathcal{T}^{(i)}\right), \hat{A}_{\sigma}\right]_{+}-2 i \mathcal{L}_{\hat{\mathfrak{g}}(\sigma)}^{0, \mu ; 0, \nu}(\sigma) \mathcal{T}_{0, \mu}^{(i)} \hat{A}_{\sigma} \mathcal{T}_{0, \nu}^{(i)} \\
& -2 i \mathcal{L}_{\hat{\mathfrak{g}}(\sigma) \mu}^{n(r) \mu ;-n(r), \nu}(\sigma)\left(\frac{\bar{n}(r)}{\rho(\sigma)} \hat{A}_{\sigma} \mathcal{T}_{n(r) \mu}^{(i)} \mathcal{T}_{-n(r), \nu}^{(i)}+\frac{\overline{-n(r)}}{\rho(\sigma)} \mathcal{T}_{n(r) \mu}^{(i)} \mathcal{T}_{-n(r), \nu}^{(i)} \hat{A}_{\sigma}\right) \\
& \phi_{i} \equiv t_{i}+\xi_{i}, \quad \bar{\phi}_{i} \equiv t_{i}-\xi_{i}, \quad i, j=1, \ldots, n .
\end{aligned}
$$

Tensor products are assumed in the result (4.10), for example

$$
\begin{gathered}
\left(\hat{A}_{\sigma} \mathcal{T}^{(i)} \mathcal{T}^{(j)}\right)_{N\left(r_{i}\right) \mu_{i}, N\left(r_{j}\right) \mu_{j}}^{N\left(s_{i} \nu_{i}, N\left(s_{j}\right) \nu_{j}\right.} \equiv\left(\hat{A}_{\sigma} \mathcal{T}^{(i)} \otimes \mathcal{T}^{(j)}\right)_{N\left(r_{i}\right) \mu_{i}, N\left(r_{j}\right) \mu_{j}}^{N\left(s_{i}\right) \nu_{i}, N\left(s_{j}\right) \nu_{j}} \\
=\left(\hat{A}_{\sigma}\right)_{N\left(r_{i}\right) \mu_{i}, N\left(r_{j}\right) \mu_{j}}^{N\left(t_{i}\right) \delta_{i}, N\left(t_{j}\right) \delta_{j}}\left(\mathcal{T}^{(i)}\right)_{N\left(t_{i}\right) \delta_{i}}{ }^{N\left(s_{i}\right) \nu_{i}}\left(\mathcal{T}^{(j)}\right)_{N\left(t_{j}\right) \delta_{j}}^{N\left(s_{j}\right) \nu_{j}}, \quad i \neq j \\
{\left[\mathcal{T}_{n(r) \mu}^{(i)}, \mathcal{T}_{n(s) \nu}^{(j)}\right]=i \delta^{i j} \mathcal{F}_{n(r) \mu ; n(s) \nu}{ }^{n(t) \delta}(\sigma) \mathcal{T}_{n(t) \delta}^{(i)}}
\end{gathered}
$$

although $\mathcal{T}^{(i)} \mathcal{T}^{(i)}$ is standard matrix multiplication.

In the derivation of Eq. (4.10), we also needed the commutators:

$$
\begin{gathered}
{\left[\hat{J}_{n(r) \mu}^{(+) \pm}\left(\xi_{i}, t_{i}\right), \hat{g}\left(\mathcal{T}^{(j)}, \xi_{j}, t_{j}\right)\right]= \pm \frac{e^{i \frac{\bar{n}(r)}{\rho(\sigma)}\left(\phi_{j}-\phi_{i}\right)}}{1-e^{i\left(\phi_{j}-\phi_{i}\right)}} \hat{g}\left(\mathcal{T}^{(j)}, \xi_{j}, t_{j}\right) \mathcal{T}_{n(r) \mu}^{(j)}} \\
\mp \frac{e^{i \frac{\bar{n}(r)}{\rho(\sigma)}\left(\bar{\phi}_{j}-\phi_{i}\right)}}{1-e^{i\left(\bar{\phi}_{j}-\phi_{i}\right)}} \mathcal{T}_{n(r) \mu}^{(j)} \hat{g}\left(\mathcal{T}^{(j)}, \xi_{j}, t_{j}\right) \\
{\left[\hat{J}_{n(r) \mu}^{(-) \pm}\left(\xi_{i}, t_{i}\right), \hat{g}\left(\mathcal{T}^{(j)}, \xi_{j}, t_{j}\right)\right]= \pm \frac{e^{i \frac{\bar{n}(r)}{\rho(\sigma)}\left(\phi_{j}-\bar{\phi}_{i}\right)}}{1-e^{i\left(\phi_{j}-\bar{\phi}_{i}\right)}} \hat{g}\left(\mathcal{T}^{(j)}, \xi_{j}, t_{j}\right) \mathcal{T}_{n(r) \mu}^{(j)}} \\
\mp \frac{e^{i \frac{\bar{n}(r)}{\rho(\sigma)}\left(\bar{\phi}_{j}-\bar{\phi}_{i}\right)}}{1-e^{i\left(\bar{\phi}_{j}-\bar{\phi}_{i}\right)}} \mathcal{T}_{n(r) \mu}^{(j)} \hat{g}\left(\mathcal{T}^{(j)}, \xi_{j}, t_{j}\right) .
\end{gathered}
$$


The infinite sums evaluated here are conditionally convergent, for example

$$
\begin{aligned}
{\left[\hat{J}_{n(r) \mu}^{(+)+}\left(\xi_{i}, t_{i}\right), \hat{g}\left(\mathcal{T}^{(j)}, \xi_{j}, t_{j}\right)\right] } & =e^{i \frac{\bar{n}(r)}{\rho(\sigma)}\left(\phi_{j}-\phi_{i}\right)} \hat{g}\left(\mathcal{T}^{(j)}, \xi_{j}, t_{j}\right) \mathcal{T}_{n(r) \mu}^{(j)} \sum_{m \geq 0} e^{i m\left(\phi_{j}-\phi_{i}\right)} \\
& -e^{i \frac{\bar{n}(r)}{\rho(\sigma)}\left(\bar{\phi}_{j}-\phi_{i}\right)} \mathcal{T}_{n(r) \mu}^{(j)} \hat{g}\left(\mathcal{T}^{(j)}, \xi_{j}, t_{j}\right) \sum_{m \geq 0} e^{i m\left(\bar{\phi}_{j}-\phi_{i}\right)}
\end{aligned}
$$

and we used the untwisted open-string prescription of Ref. 23] to evaluate these sums.

The global Ward identities in Eq. (4.8) and the differential equations in Eq. (4.10) are the first two components of the desired open-string $\mathrm{KZ}$ system for $A_{g}^{\text {open }}(H) / H$, a complete form of which can be found in Subsec. 4.6.

\subsection{Constituent Twisted Affine Primary Fields}

In this subsection, we introduce the constituent twisted affine primary fields which provide an alternate derivation of the twisted vertex operator equation (4.4b) for $\partial_{t} \hat{g}$, and will also be helpful in determining the corresponding twisted vertex operator equation for $\partial_{\xi} \hat{g}$.

Following Refs. [42, 43, 23, 6, 9], we define the constituent twisted affine primary fields $\hat{g}_{ \pm}(\mathcal{T})$ by factorization of the twisted affine primary fields:

$$
\begin{aligned}
\hat{g}(\mathcal{T}, \xi, t) & \equiv \hat{g}_{-}(\mathcal{T}, \xi, t) \hat{g}_{+}(\mathcal{T}, \xi, t) \\
{\left[\hat{J}_{n(r) \mu}\left(m+\frac{n(r)}{\rho(\sigma)}\right), \hat{g}_{+}(\mathcal{T}, \xi, t)\right] } & =\hat{g}_{+}(\mathcal{T}, \xi, t) \mathcal{T}_{n(r) \mu} e^{i\left(m+\frac{n(r)}{\rho(\sigma)}\right)(t+\xi)} \\
{\left[\hat{J}_{n(r) \mu}\left(m+\frac{n(r)}{\rho(\sigma)}\right), \hat{g}_{-}(\mathcal{T}, \xi, t)\right] } & =-\mathcal{T}_{n(r) \mu} \hat{g}_{-}(\mathcal{T}, \xi, t) e^{i\left(m+\frac{n(r)}{\rho(\sigma)}\right)(t-\xi)}
\end{aligned}
$$

The simpler commutators in Eq. (4.14b,c), reminiscent of chiral primary fields, reproduce the algebra (4.3) of the current modes with the full twisted affine primary fields $\hat{g}(\mathcal{T})$.

By direct computation with Eq. (4.14) and the mode form (4.1a) of the Hamiltonian, we find after some algebra the twisted vertex operator equations of the constituents

$$
\begin{aligned}
& \partial_{t} \hat{g}_{ \pm}(\mathcal{T}, \xi, t)=i\left[\hat{H}_{\sigma}, \hat{g}_{ \pm}(\mathcal{T}, \xi, t)\right] \\
& \partial_{t} \hat{g}_{+}(\mathcal{T}, \xi, t)=2 i \mathcal{L}_{\hat{\mathfrak{g}}(\sigma)}^{n(r) \mu ;-n(r), \nu}(\sigma): \hat{J}_{n(r) \mu}^{(+)}(\xi, t) \hat{g}_{+}(\mathcal{T}, \xi, t) \mathcal{T}_{-n(r), \nu}:_{M} \\
& +i \hat{g}_{+}(\mathcal{T}, \xi, t) \mathcal{D}_{\hat{\mathfrak{g}}(\sigma)}(\mathcal{T})-2 i \mathcal{L}_{\hat{\mathfrak{g}}(\sigma)}^{n(r) \mu ;-n(r), \nu}(\sigma) \frac{\bar{n}(r)}{\rho(\sigma)} \hat{g}_{+}(\mathcal{T}, \xi, t) \mathcal{T}_{n(r) \mu} \mathcal{T}_{-n(r), \nu} \\
& \partial_{t} \hat{g}_{-}(\mathcal{T}, \xi, t)=-2 i \mathcal{L}_{\hat{\mathfrak{g}}(\sigma)}^{n(r) \mu ;-n(r), \nu}(\sigma): \mathcal{T}_{n(r) \mu} \hat{J}_{-n(r), \nu}^{(-)}(\xi, t) \hat{g}_{-}(\mathcal{T}, \xi, t):_{M} \\
& +i \mathcal{D}_{\hat{\mathfrak{g}}(\sigma)}(\mathcal{T}) \hat{g}_{-}(\mathcal{T}, \xi, t)-2 i \mathcal{L}_{\hat{\mathfrak{g}}(\sigma)}^{n(r) \mu ;-n(r), \nu}(\sigma) \overline{\overline{-n(r)}} \frac{\bar{\rho}(\sigma)}{\mathcal{T}_{n(r) \mu}} \mathcal{T}_{-n(r), \nu} \hat{g}_{-}(\mathcal{T}, \xi, t)
\end{aligned}
$$


where we have again used the identities in Eq. (4.7) to simplify these results. The mode normal ordering here is the same as that defined in Eq. (4.5) with $\hat{g} \rightarrow \hat{g}_{ \pm}$.

As a check on the consistency of the factorization (4.14), we may use Eqs. (4.15b,c) to successfully reconstruct the vertex operator equation (4.6) of the full twisted affine primary field:

$$
\begin{aligned}
\partial_{t} \hat{g}( & \mathcal{T}, \xi, t)=\partial_{t} \hat{g}_{-}(\mathcal{T}, \xi, t) \hat{g}_{+}(\mathcal{T}, \xi, t)+\hat{g}_{-}(\mathcal{T}, \xi, t) \partial_{t} \hat{g}_{+}(\mathcal{T}, \xi, t) \\
= & 2 i \mathcal{L}_{\hat{\mathfrak{g}}(\sigma) \mu}^{n(r) \mu ;-n(r), \nu}(\sigma)\left(\hat{g}_{-}(\mathcal{T}, \xi, t): \hat{J}_{n(r) \mu}^{(+)}(\xi, t) \hat{g}_{+}(\mathcal{T}, \xi, t) \mathcal{T}_{-n(r), \nu}:_{M}\right. \\
& \left.-: \mathcal{T}_{n(r) \mu} \hat{J}_{-n(r), \nu}^{(-)}(\xi, t) \hat{g}_{-}(\mathcal{T}, \xi, t):_{M} \hat{g}_{+}(\mathcal{T}, \xi, t)\right)+i\left[\mathcal{D}_{\hat{\mathfrak{g}}(\sigma)}(\mathcal{T}), \hat{g}(\mathcal{T}, \xi, t)\right]_{+} \\
& -2 i \mathcal{L}_{\hat{\mathfrak{g}}(\sigma) \mu}^{n(r) \mu ;-n(r), \nu}(\sigma)\left(\frac{\bar{n}(r)}{\rho(\sigma)} \hat{g}(\mathcal{T}, \xi, t) \mathcal{T}_{n(r) \mu} \mathcal{T}_{-n(r), \nu}+\overline{\frac{-n(r)}{\rho(\sigma)}} \mathcal{T}_{n(r) \mu} \mathcal{T}_{-n(r), \nu} \hat{g}(\mathcal{T}, \xi, t)\right) \\
= & 2 i \mathcal{L}_{\hat{\mathfrak{g}}(\sigma)}^{n(r) \mu ;-n(r), \nu}(\sigma)\left(: \hat{J}_{n(r) \mu}^{(+)}(\xi, t) \hat{g}(\mathcal{T}, \xi, t) \mathcal{T}_{-n(r), \nu}-\mathcal{T}_{n(r) \mu} \hat{J}_{-n(r), \nu}^{(-)}(\xi, t) \hat{g}(\mathcal{T}, \xi, t):_{M}\right) \\
& +i\left[\mathcal{D}_{\hat{\mathfrak{g}}(\sigma)}(\mathcal{T}), \hat{g}(\mathcal{T}, \xi, t)\right]_{+}-2 i \mathcal{L}_{\hat{\mathfrak{g}}(\sigma)}^{0, \mu ; 0, \nu}(\sigma) \mathcal{T}_{0, \mu} \hat{g}(\mathcal{T}, \xi, t) \mathcal{T}_{0, \nu} \\
& -2 i \mathcal{L}_{\hat{\mathfrak{g}}(\sigma)}^{n(r) \mu ;-n(r), \nu}(\sigma)\left(\frac{\bar{n}(r)}{\rho(\sigma)} \hat{g}(\mathcal{T}, \xi, t) \mathcal{T}_{n(r) \mu} \mathcal{T}_{-n(r), \nu}+\frac{\overline{-n(r)}}{\rho(\sigma)} \mathcal{T}_{n(r) \mu} \mathcal{T}_{-n(r), \nu} \hat{g}(\mathcal{T}, \xi, t)\right)
\end{aligned}
$$

In this computation, the expression in Eq. (4.16b) was not completely normal-ordered, so we used the following identities

$$
\begin{gathered}
{\left[\hat{J}_{n(r) \mu}^{(+)-}(\xi, t), \hat{g}_{-}(\mathcal{T}, \xi, t)\right]=\frac{e^{-2 i \frac{\bar{n}(r)}{\rho(\sigma)} \xi}}{1-e^{-2 i \xi}} \mathcal{T}_{n(r) \mu} \hat{g}_{-}(\mathcal{T}, \xi, t)} \\
{\left[\hat{J}_{n(r) \mu}^{(-)+}(\xi, t), \hat{g}_{+}(\mathcal{T}, \xi, t)\right]=\frac{e^{2 i \frac{\bar{n}(r)}{\rho(\sigma)} \xi}}{1-e^{2 i \xi}} \hat{g}_{+}(\mathcal{T}, \xi, t) \mathcal{T}_{n(r) \mu}} \\
\frac{e^{2 i \frac{\overline{-n(r)}}{\rho(\sigma)} \xi}}{1-e^{2 i \xi}}+\frac{e^{-2 i \frac{\bar{n}(r)}{\rho(\sigma)} \xi}}{1-e^{-2 i \xi}}=\delta_{\bar{n}(r), 0}
\end{gathered}
$$

to obtain the completely normal-ordered result in Eq. 4.16c). With Ref. 23, we note the singularities at $\xi=0$ and $\pi$ of the commutators in Eq. (4.17). Although (as seen in Eq. (4.17c) ) these singularities cancel in the computation of $\partial_{t} \hat{g}$, we shall see in Subsec. 4.5 that the singularities persist in $\partial_{\xi} \hat{g}$.

Using the mode-ordered form (A.3a), the algebra (4.14) and the twisted vertex operator equations, we may also give the action of the Virasoro generators $L_{\sigma}(m)$ on the constituent twisted affine primary fields:

$$
\begin{aligned}
& {\left[L_{\sigma}(m), \hat{g}_{+}(\mathcal{T}, \xi, t)\right]=\hat{g}_{+}(\mathcal{T}, \xi, t)\left(-i \overleftarrow{\partial}_{t}+m \mathcal{D}_{\hat{\mathfrak{g}}(\sigma)}(\mathcal{T})\right) e^{i m(t+\xi)}} \\
& {\left[L_{\sigma}(m), \hat{g}_{-}(\mathcal{T}, \xi, t)\right]=e^{i m(t-\xi)}\left(-i \partial_{t}+m \mathcal{D}_{\hat{\mathfrak{g}}(\sigma)}(\mathcal{T})\right) \hat{g}_{-}(\mathcal{T}, \xi, t)}
\end{aligned}
$$

We have checked that these commutators satisfy the $L, L, \hat{g}_{ \pm}$Jacobi identities. 


\subsection{The Constituents are Chiral}

In the untwisted results of Ref. [23, the constituent affine primary fields $g_{+}(T), g_{-}(T)$ of $A_{g}^{\text {open }}$ are in fact chiral and anti-chiral respectively. As we will see below, the same properties hold for the twisted constituent fields $\hat{g}_{+}(\mathcal{T}), \hat{g}_{-}(\mathcal{T})$ of the open-string orbifold $A_{g}^{\text {open }}(H) / H$.

Following Ref. [23], we begin this discussion by returning to the bulk momentum operator (2.17)

$$
\hat{P}_{\sigma}(t)=-\frac{2 i}{\pi} \sum_{m \in \mathbb{Z}} \frac{e^{-i(2 m+1) t}}{2 m+1} L_{\sigma}(2 m+1)
$$

which we have now expressed in terms of the open-string Virasoro modes. By direct computation with the algebra (4.18), we then find that

$$
\begin{aligned}
i\left[\hat{P}_{\sigma}(t), \hat{g}_{+}(\mathcal{T}, \xi, t)\right]= & \left(\int_{0}^{\xi} d \eta e^{i \eta} \delta(2 \eta)\right) \partial_{t} \hat{g}_{+}(\mathcal{T}, \xi, t) \\
& +4 e^{i \xi} \delta(2 \xi) \hat{g}_{+}(\mathcal{T}, \xi, t) \mathcal{D}_{\hat{\mathfrak{g}}(\sigma)}(\mathcal{T}) \\
i\left[\hat{P}_{\sigma}(t), \hat{g}_{-}(\mathcal{T}, \xi, t)\right]= & -4\left(\int_{0}^{\xi} d \eta e^{-i \eta} \delta(2 \eta)\right) \partial_{t} \hat{g}_{-}(\mathcal{T}, \xi, t) \\
& +4 e^{-i \xi} \delta(2 \xi) \mathcal{D}_{\hat{\mathfrak{g}}(\sigma)}(\mathcal{T}) \hat{g}_{-}(\mathcal{T}, \xi, t) \\
4 \int_{0}^{\xi} d \eta e^{i \eta} \delta(2 \eta)= & 4 \int_{0}^{\xi} d \eta e^{-i \eta} \delta(2 \eta)= \begin{cases}1 & \text { if } 0<\xi<\pi, \\
0 & \text { if } \xi=0, \pi\end{cases}
\end{aligned}
$$

where the twisted conformal weight matrix $\mathcal{D}_{\hat{\mathfrak{g}}(\sigma)}(\mathcal{T})$ is defined in Eq. (4.6b). The summation identities 23]

$$
\sum_{m \in \mathbb{Z}} \frac{e^{ \pm i(2 m+1) \xi}}{2 m+1}= \pm 2 \pi i \int_{0}^{\xi} d \eta e^{ \pm i \eta} \delta(2 \eta)
$$

were used to obtain these results.

The last terms in Eqs. (4.20a) and (4.20b) are quantum effects which contribute only at the boundary, so that the result

$$
\partial_{\xi} \hat{g}_{ \pm}(\mathcal{T}, \xi, t)=i\left[\hat{P}_{\sigma}(t), \hat{g}_{ \pm}(\mathcal{T}, \xi, t)\right]= \pm \partial_{t} \hat{g}(\mathcal{T}, \xi, t) \quad 0<\xi<\pi
$$

is obtained in the bulk. Following the classical intuition developed in Subsec. 3.2, we may smoothly extend this result to include the boundary

$$
\partial_{-} \hat{g}_{+}(\mathcal{T}, \xi, t)=\partial_{+} \hat{g}_{-}(\mathcal{T}, \xi, t)=0, \quad \partial_{ \pm}=\partial_{t} \pm \partial_{\xi}, \quad 0 \leq \xi \leq \pi
$$


which tells us that, as in Ref. [23], the operators $\hat{g}_{+}(\mathcal{T})$ and $\hat{g}_{-}(\mathcal{T})$ are respectively chiral and anti-chiral.

In fact, we may use the twisted vertex operator equations (4.15) and the chiralities (4.23) in the form $\frac{1}{2} \partial_{ \pm} \hat{g}_{ \pm}=\partial_{t} \hat{g}_{ \pm}$to obtain the following set of light-cone twisted vertex operator equations:

$$
\begin{aligned}
& \frac{1}{2} \partial_{+} \hat{g}_{+}(\mathcal{T}, \xi, t)=2 i \mathcal{L}_{\hat{\mathfrak{g}}(\sigma)}^{n(r) \mu ;-n(r), \nu}(\sigma): \hat{J}_{n(r) \mu}^{(+)}(\xi, t) \hat{g}_{+}(\mathcal{T}, \xi, t) \mathcal{T}_{-n(r), \nu}:_{M} \\
& \quad+i \hat{g}_{+}(\mathcal{T}, \xi, t) \mathcal{D}_{\hat{\mathfrak{g}}(\sigma)}(\mathcal{T})-2 i \mathcal{L}_{\hat{\mathfrak{g}}(\sigma)}^{n(r) \mu ;-n(r), \nu}(\sigma) \frac{\bar{n}(r)}{\rho(\sigma)} \hat{g}_{+}(\mathcal{T}, \xi, t) \mathcal{T}_{n(r) \mu} \mathcal{T}_{-n(r), \nu} \\
& \frac{1}{2} \partial_{-} \hat{g}_{-}(\mathcal{T}, \xi, t)=-2 i \mathcal{L}_{\hat{\mathfrak{g}}(\sigma)}^{n(r) \mu ;-n(r), \nu}(\sigma): \mathcal{T}_{n(r) \mu} \hat{J}_{-n(r), \nu}^{(-)}(\xi, t) \hat{g}_{-}(\mathcal{T}, \xi, t):_{M} \\
& \quad+i \mathcal{D}_{\hat{\mathfrak{g}}(\sigma)}(\mathcal{T}) \hat{g}_{-}(\mathcal{T}, \xi, t)-2 i \mathcal{L}_{\hat{\mathfrak{g}}(\sigma)}^{n(r) \mu ;-n(r), \nu}(\sigma) \frac{\overline{-n(r)}}{\rho(\sigma)} \mathcal{T}_{n(r) \mu} \mathcal{T}_{-n(r), \nu} \hat{g}_{-}(\mathcal{T}, \xi, t)
\end{aligned}
$$

It is easily checked that these equations are consistent

$$
\partial_{+} \partial_{-} \hat{g}_{ \pm}(\mathcal{T}, \xi, t)=\partial_{-} \partial_{+} \hat{g}_{ \pm}(\mathcal{T}, \xi, t)=0
$$

because $\hat{g}_{+}(\mathcal{T}), \hat{J}^{(+)}(\xi)$ are chiral and $\hat{g}_{-}(\mathcal{T}), \hat{J}^{(-)}(\xi)$ are anti-chiral.

Together, the chirality conditions (4.23) and the light-cone vertex operator equations (4.24) will determine the dynamics of the full open-string primary fields $\hat{g}=\hat{g}_{-} \hat{g}_{+}$. We defer this analysis to the following subsection however, focussing here on further properties of the constituent fields.

As an example, we may use the differential equations (4.24) and the commutator identities

$$
\begin{aligned}
& {\left[\hat{J}_{n(r) \mu}^{(+) \pm}\left(\xi_{i}, t_{i}\right), \hat{g}_{+}\left(\mathcal{T}^{(j)}, \xi_{j}, t_{j}\right)\right]= \pm \frac{e^{i \frac{\bar{n}(r)}{\rho(\sigma)}\left(\phi_{j}-\phi_{i}\right)}}{1-e^{i\left(\phi_{j}-\phi_{i}\right)}} \hat{g}_{+}\left(\mathcal{T}^{(j)}, \xi_{j}, t_{j}\right) \mathcal{T}_{n(r) \mu}^{(j)}} \\
& {\left[\hat{J}_{n(r) \mu}^{(-) \pm}\left(\xi_{i}, t_{i}\right), \hat{g}_{-}\left(\mathcal{T}^{(j)}, \xi_{j}, t_{j}\right)\right]=\mp \frac{e^{i \frac{\bar{n}(r)}{\rho\left(\bar{\rho}_{j}-\bar{\phi}_{i}\right)}}}{1-e^{i\left(\bar{\phi}_{j}-\bar{\phi}_{i}\right)}} \mathcal{T}_{n(r) \mu}^{(j)} \hat{g}_{-}\left(\mathcal{T}^{(j)}, \xi_{j}, t_{j}\right)}
\end{aligned}
$$

to find the twisted KZ equations for the chiral and anti-chiral correlators $\hat{A}_{\sigma}^{ \pm}$:

$$
\begin{gathered}
\hat{g}_{ \pm}(i) \equiv \hat{g}_{ \pm}\left(\mathcal{T}^{(i)}, \xi_{i}, t_{i}\right), \quad \hat{A}_{\sigma}^{ \pm} \equiv\left\langle\hat{g}_{ \pm}(1) \cdots \hat{g}_{ \pm}(n)\right\rangle_{\sigma}, \quad \partial_{i-} \hat{A}_{\sigma}^{+}=\partial_{i+} \hat{A}_{\sigma}^{-}=0 \\
\frac{1}{2} \partial_{i+} \hat{A}_{\sigma}^{+}=2 i \mathcal{L}_{\hat{\mathfrak{g}}(\sigma)}^{n(r) \mu ;-n(r), \nu}(\sigma) \sum_{j \neq i} \frac{e^{i \frac{\bar{n}(r)}{\rho(\sigma)}\left(\phi_{j}-\phi_{i}\right)}}{1-e^{i\left(\phi_{j}-\phi_{i}\right)}} \hat{A}_{\sigma}^{+} \mathcal{T}_{n(r) \mu}^{(j)} \mathcal{T}_{-n(r), \nu}^{(i)} \\
+i \hat{A}_{\sigma}^{+} \mathcal{D}_{\hat{\mathfrak{g}}(\sigma)}\left(\mathcal{T}^{(i)}\right)-2 i \mathcal{L}_{\hat{\mathfrak{g}}(\sigma)}^{n(r) \mu ;-n(r), \nu}(\sigma) \frac{\bar{n}(r)}{\rho(\sigma)} \hat{A}_{\sigma}^{+} \mathcal{T}_{n(r) \mu}^{(i)} \mathcal{T}_{-n(r), \nu}^{(i)}
\end{gathered}
$$




$$
\begin{gathered}
\frac{1}{2} \partial_{i-} \hat{A}_{\sigma}^{-}=2 i \mathcal{L}_{\hat{\mathfrak{g}}(\sigma)}^{n(r) \mu ;-n(r), \nu}(\sigma) \sum_{j \neq i} \frac{e^{i \frac{\bar{n}(r)}{\rho\left(\bar{\phi}_{j}-\bar{\phi}_{i}\right)}}}{1-e^{i\left(\bar{\phi}_{j}-\bar{\phi}_{i}\right)}} \mathcal{T}_{-n(r), \nu}^{(i)} \mathcal{T}_{n(r) \mu}^{(j)} \hat{A}_{\sigma}^{-} \\
+i \mathcal{D}_{\hat{\mathfrak{g}}(\sigma)}\left(\mathcal{T}^{(i)}\right) \hat{A}_{\sigma}^{-}-2 i \mathcal{L}_{\hat{\mathfrak{g}}(\sigma)}^{n(r) \mu ;-n(r), \nu}(\sigma) \overline{\frac{-n(r)}{\rho(\sigma)}} \mathcal{T}_{n(r) \mu}^{(i)} \mathcal{T}_{-n(r), \nu}^{(i)} \hat{A}_{\sigma}^{-} \\
\partial_{i \pm} \equiv \partial_{t_{i}} \pm \partial_{\xi_{i}}, \quad \mathcal{T}^{(i)}=\mathcal{T}\left(T^{(i)}\right), \quad i=1 \ldots n
\end{gathered}
$$

These twisted KZ equations are similar in form to the chiral and anti-chiral twisted KZ equations of closed-string orbifold theory [6, 7, 9, 10, In contrast to closed-string orbifold theory, however, the open-string correlators $\hat{A}_{\sigma}$ of the full twisted affine primary fields cannot be factorized into the open-string chiral and anti-chiral correlators $\hat{A}_{\sigma}^{ \pm}$:

$$
\begin{gathered}
\hat{g}(i) \equiv \hat{g}\left(\mathcal{T}^{(i)}, \xi_{i}, t_{i}\right)=\hat{g}_{-}(i) \hat{g}_{+}(i) \\
\hat{A}_{\sigma}=\langle\hat{g}(1) \cdots \hat{g}(n)\rangle_{\sigma} \neq\left\langle\hat{g}_{-}(1) \cdots \hat{g}_{-}(n)\right\rangle_{\sigma}\left\langle\hat{g}_{+}(1) \cdots \hat{g}_{+}(n)\right\rangle_{\sigma}=\hat{A}_{\sigma}^{-} \hat{A}_{\sigma}^{+} .
\end{gathered}
$$

This follows because in open WZW theory [23], twisted or untwisted, the single set of current modes $\hat{J}$ (or $J$ ) acts on both chiral constituents of the primary fields, so that the chiral constituents are not independent.

Finally, we may use the chirality (4.23) to recast the commutators (4.18) in the form

$$
\begin{aligned}
& {\left[L_{\sigma}(m), \hat{g}_{+}(\mathcal{T}, \xi, t)\right]=\hat{g}_{+}(\mathcal{T}, \xi, t)\left(-\frac{i}{2} \overleftarrow{\partial}_{+}+m \mathcal{D}_{\hat{\mathfrak{g}}(\sigma)}(\mathcal{T})\right) e^{i m(t+\xi)}} \\
& {\left[L_{\sigma}(m), \hat{g}_{-}(\mathcal{T}, \xi, t)\right]=e^{i m(t-\xi)}\left(-\frac{i}{2} \partial_{-}+m \mathcal{D}_{\hat{\mathfrak{g}}(\sigma)}(\mathcal{T})\right) \hat{g}_{-}(\mathcal{T}, \xi, t)}
\end{aligned}
$$

and these commutators also satisfy the $L, L, \hat{g}_{ \pm}$Jacobi identities, as expected.

\subsection{The Full Twisted Vertex Operator Equations}

In this subsection, we assemble the chiral and anti-chiral constituent information above to obtain the complete set of vertex operator equations $\partial_{t} \hat{g}, \partial_{\xi} \hat{g}$ for the full twisted affine primary fields $\hat{g}$ of the open-string orbifold.

We begin by writing down the following set of light-cone twisted vertex operator equations for $\hat{g}$ :

$$
\begin{aligned}
\frac{1}{2} \partial_{+} \hat{g}(\mathcal{T}, \xi, t)=2 i \mathcal{L}_{\hat{\mathfrak{g}}(\sigma)}^{n(r) \mu-n(r), \nu}(\sigma): \hat{J}_{n(r) \mu}^{(+)}(\xi, t) \hat{g}(\mathcal{T}, \xi, t) \mathcal{T}_{-n(r), \nu}:_{M} \\
\quad+i \hat{g}(\mathcal{T}, \xi, t) \mathcal{D}_{\hat{\mathfrak{g}}(\sigma)}(\mathcal{T})-2 i \mathcal{L}_{\hat{\mathfrak{g}}(\sigma)}^{n(r) \mu ;-n(r), \nu}(\sigma) \frac{\bar{n}(r)}{\rho(\sigma)} \hat{g}(\mathcal{T}, \xi, t) \mathcal{T}_{n(r) \mu} \mathcal{T}_{-n(r), \nu} \\
\quad-2 i \mathcal{L}_{\hat{\mathfrak{g}}(\sigma) \mu ;-n(r), \nu}^{n(r)}(\sigma) \frac{e^{-2 i \frac{\bar{n}(r)}{\rho(\sigma)} \xi}}{1-e^{-2 i \xi}} \mathcal{T}_{n(r) \mu} \hat{g}(\mathcal{T}, \xi, t) \mathcal{T}_{-n(r), \nu}
\end{aligned}
$$




$$
\begin{aligned}
\frac{1}{2} \partial_{-} \hat{g}(\mathcal{T}, \xi, t)=-2 i \mathcal{L}_{\hat{\mathfrak{g}}(\sigma)}^{n(r) \mu ;-n(r), \nu}(\sigma): \mathcal{T}_{n(r) \mu} \hat{J}_{-n(r), \nu}^{(-)}(\xi, t) \hat{g}(\mathcal{T}, \xi, t):_{M} \\
\quad+i \mathcal{D}_{\hat{\mathfrak{g}}(\sigma)}(\mathcal{T}) \hat{g}(\mathcal{T}, \xi, t)-2 i \mathcal{L}_{\hat{\mathfrak{g}}(\sigma)}^{n(r) \mu ;-n(r), \nu}(\sigma) \frac{\overline{-n(r)}}{\rho(\sigma)} \mathcal{T}_{n(r) \mu} \mathcal{T}_{-n(r), \nu} \hat{g}(\mathcal{T}, \xi, t) \\
\quad-2 i \mathcal{L}_{\hat{\mathfrak{g}}(\sigma)}^{n(r) \mu ;-n(r), \nu}(\sigma) \frac{e^{2 i \frac{\overline{-n(r)}}{\rho(\sigma)} \xi}}{1-e^{2 i \xi}} \mathcal{T}_{n(r) \mu} \hat{g}(\mathcal{T}, \xi, t) \mathcal{T}_{-n(r), \nu}
\end{aligned}
$$

To obtain these equations, we used the chiralities (4.23) and the constituent vertex operator equations (4.24) - and moreover, as in Subsec. 4.3, the commutators (4.17) were used to obtain the fully normal-ordered form given here. The consistency of this system

$$
\left[\partial_{+}, \partial_{-}\right] \hat{g}(\mathcal{T}, \xi, t)=0, \quad \partial_{ \pm}=\partial_{t} \pm \partial_{\xi}
$$

follows by construction from $\hat{g}=\hat{g}_{-} \hat{g}_{+}$.

Using the light-cone vertex operator equations (4.31), we then immediately obtain the vertex operator equations for the $t$ - and $\xi$-derivatives separately:

$$
\begin{aligned}
\partial_{t} \hat{g} & (\mathcal{T}, \xi, t)= \\
= & \left.2 i \mathcal{L}_{\hat{\mathfrak{g}}(\sigma) \mu ;-n(r), \nu}^{n(r)}(\sigma) \hat{J}_{n(r) \mu}^{(+)}(\xi, t) \hat{g}(\mathcal{T}, \xi, t) \mathcal{T}_{-n(r), \nu}-\mathcal{T}_{n(r) \mu} \hat{J}_{-n(r), \nu}^{(-)}(\xi, t) \hat{g}(\mathcal{T}, \xi, t):_{M}\right) \\
& +i\left[\mathcal{D}_{\hat{\mathfrak{g}}(\sigma)}(\mathcal{T}), \hat{g}(\mathcal{T}, \xi, t)\right]_{+}-2 i \mathcal{L}_{\hat{\mathfrak{g}}(\sigma)}^{0, \mu ; 0, \nu}(\sigma) \mathcal{T}_{0, \mu} \hat{g}(\mathcal{T}, \xi, t) \mathcal{T}_{0, \nu} \\
& -2 i \mathcal{L}_{\hat{\mathfrak{g}}(\sigma)}^{n(r) \mu ;-n(r), \nu}(\sigma)\left(\frac{\bar{n}(r)}{\rho(\sigma)} \hat{g}(\mathcal{T}, \xi, t) \mathcal{T}_{n(r) \mu} \mathcal{T}_{-n(r), \nu}+\frac{\overline{-n(r)}}{\rho(\sigma)} \mathcal{T}_{n(r) \mu} \mathcal{T}_{-n(r), \nu} \hat{g}(\mathcal{T}, \xi, t)\right) \\
\partial_{\xi} \hat{g}(\mathcal{T}, \xi, t)= & \\
= & 2 i \mathcal{L}_{\hat{\mathfrak{g}}(\sigma)}^{n(r) \mu ;-n(r), \nu}(\sigma)\left(: \hat{J}_{n(r) \mu}^{(+)}(\xi, t) \hat{g}(\mathcal{T}, \xi, t) \mathcal{T}_{-n(r), \nu}+\mathcal{T}_{n(r) \mu} \hat{J}_{-n(r), \nu}^{(-)}(\xi, t) \hat{g}(\mathcal{T}, \xi, t):_{M}\right) \\
& +i\left[\mathcal{D}_{\hat{\mathfrak{g}}(\sigma)}(\mathcal{T}), \hat{g}(\mathcal{T}, \xi, t)\right] \\
& +2 i \mathcal{L}_{\hat{\mathfrak{g}}(\sigma)}^{n(r) \mu ;-n(r), \nu}(\sigma)\left(i \cot \xi \delta_{\bar{n}(r), 0}-\frac{2 e^{-2 i \frac{\bar{n}(r)}{\rho(\sigma)} \xi}}{1-e^{-2 i \xi}}\left(1-\delta_{\bar{n}(r), 0}\right)\right) \mathcal{T}_{n(r) \mu} \hat{g}(\mathcal{T}, \xi, t) \mathcal{T}_{-n(r), \nu} \\
& -2 i \mathcal{L}_{\hat{\mathfrak{g}}(\sigma)}^{n(r) \mu-n(r), \nu}(\sigma)\left(\frac{\bar{n}(r)}{\rho(\sigma)} \hat{g}(\mathcal{T}, \xi, t) \mathcal{T}_{n(r) \mu} \mathcal{T}_{-n(r), \nu}-\frac{\overline{-n(r)}}{\rho(\sigma)} \mathcal{T}_{n(r) \mu} \mathcal{T}_{-n(r), \nu} \hat{g}(\mathcal{T}, \xi, t)\right)
\end{aligned}
$$

We remind that the $\partial_{t}$ equation appeared earlier in Eq. (4.16c).

As anticipated in Subsec. 4.3, the singularities in Eq. $(4.17 \mathrm{a}, \mathrm{b})$ at $\xi=0$ and $\pi$ persist in the $\partial_{\xi} \hat{g}$ equation (4.33b) - and hence in the twisted affine primary fields themselves. Following Ref. 23], we emphasize that these singularities are closely related to the nonfactorization phenomenon in Eq. (4.29): Both phenomena arise because the chiral constituents $\hat{g}_{+}$and $\hat{g}_{-}$of open WZW strings do not live in independent subspaces. On the 
other hand, the affine primary fields of closed strings (including ordinary orbifolds) have independent chiral constituents $\hat{g}_{ \pm}-$and therefore have no such singularities.

We finally note that the chiral commutators in Eq. (4.30) and the factorization (4.14) give the following commutator for the full twisted affine primary fields

$$
\begin{aligned}
{\left[L_{\sigma}(m), \hat{g}(\mathcal{T}, \xi, t)\right]=\hat{g}(\mathcal{T}, \xi, t)\left(-\frac{i}{2} \overleftarrow{\partial}_{+}+m \mathcal{D}_{\hat{\mathfrak{g}}(\sigma)}(\mathcal{T})\right) e^{i m(t+\xi)} } & +e^{i m(t-\xi)}\left(-\frac{i}{2} \partial_{-}+m \mathcal{D}_{\hat{\mathfrak{g}}(\sigma)}(\mathcal{T})\right) \hat{g}(\mathcal{T}, \xi, t) \\
=e^{i m t}\left(-i \cos (m \xi) \partial_{t}+\right. & \left.\sin (m \xi) \partial_{\xi}\right) \hat{g}(\mathcal{T}, \xi, t) \\
& +m e^{i m t}\left(e^{i m \xi} \hat{g}(\mathcal{T}, \xi, t) \mathcal{D}_{\hat{\mathfrak{g}}(\sigma)}(\mathcal{T})+e^{-i m \xi} \mathcal{D}_{\hat{\mathfrak{g}}(\sigma)}(\mathcal{T}) \hat{g}(\mathcal{T}, \xi, t)\right)
\end{aligned}
$$

where the twisted conformal weight matrix $\mathcal{D}_{\hat{\mathfrak{g}}(\sigma)}(\mathcal{T})$ was given in Eq. (4.6b).

\subsection{The Twisted Open-String KZ Systems of $A_{g}^{\text {open }}(H) / H$}

Using the light-cone twisted vertex operator equations (4.31) and the commutators (4.12), we may now obtain the full twisted open-string KZ system

$$
\begin{aligned}
& \hat{A}_{\sigma}={ }_{\sigma}\left\langle 0\left|\hat{g}\left(\mathcal{T}^{(1)}, \xi_{1}, t_{1}\right) \ldots \hat{g}\left(\mathcal{T}^{(n)}, \xi_{n}, t_{n}\right)\right| 0\right\rangle_{\sigma}, \quad \sigma=0, \ldots, N_{c}-1 \\
& \frac{1}{2} \partial_{+i} \hat{A}_{\sigma}=2 i \mathcal{L}_{\hat{\mathfrak{g}}(\sigma)}^{n(r) \mu-n(r), \nu}(\sigma)\left(\sum_{j \neq i} \frac{e^{i \frac{\bar{n}(r)}{\rho(\sigma)}\left(\phi_{j}-\phi_{i}\right)}}{1-e^{i\left(\phi_{j}-\phi_{i}\right)}} \hat{A}_{\sigma} \mathcal{T}_{n(r) \mu}^{(j)} \mathcal{T}_{-n(r), \nu}^{(i)}\right. \\
& \left.-\sum_{j} \frac{e^{i \frac{\bar{n}(r)}{\rho\left(\bar{\rho}_{j}-\phi_{i}\right)}}}{1-e^{i\left(\bar{\phi}_{j}-\phi_{i}\right)}} \mathcal{T}_{n(r) \mu}^{(j)} \hat{A}_{\sigma} \mathcal{T}_{-n(r), \nu}^{(i)}-\frac{\bar{n}(r)}{\rho(\sigma)} \hat{A}_{\sigma} \mathcal{T}_{n(r) \mu}^{(i)} \mathcal{T}_{-n(r), \nu}^{(i)}\right)+i \hat{A}_{\sigma} \mathcal{D}_{\hat{\mathfrak{g}}(\sigma)}\left(\mathcal{T}^{(i)}\right) \\
& \frac{1}{2} \partial_{-i} \hat{A}_{\sigma}=2 i \mathcal{L}_{\hat{\mathfrak{g}}(\sigma)}^{n(r) \mu ;-n(r), \nu}(\sigma)\left(\sum_{j \neq i} \frac{e^{i \frac{\bar{n}(r)}{\rho\left(\bar{\rho}^{\prime}\right)}\left(\bar{\phi}_{j}-\bar{\phi}_{i}\right)}}{1-e^{i\left(\bar{\phi}_{j}-\bar{\phi}_{i}\right)}} \mathcal{T}_{-n(r), \nu}^{(i)} \mathcal{T}_{n(r) \mu}^{(j)} \hat{A}_{\sigma}\right. \\
& \left.-\sum_{j} \frac{e^{i \frac{\bar{n}(r)}{\rho(\sigma)}\left(\phi_{j}-\bar{\phi}_{i}\right)}}{1-e^{i\left(\phi_{j}-\bar{\phi}_{i}\right)}} \mathcal{T}_{-n(r), \nu}^{(i)} \hat{A}_{\sigma} \mathcal{T}_{n(r) \mu}^{(j)}-\frac{\overline{-n(r)}}{\rho(\sigma)} \mathcal{T}_{n(r) \mu}^{(i)} \mathcal{T}_{-n(r), \nu}^{(i)} \hat{A}_{\sigma}\right)+i \mathcal{D}_{\hat{\mathfrak{g}}(\sigma)}\left(\mathcal{T}^{(i)}\right) \hat{A}_{\sigma} \\
& \hat{A}_{\sigma} \sum_{i=1}^{n} \mathcal{T}_{0, \mu}^{(i)}-\sum_{i=1}^{n} \mathcal{T}_{0, \mu}^{(i)} \hat{A}_{\sigma}=0 \\
& \partial_{ \pm i}=\partial_{t_{i}} \pm \partial_{\xi_{i}}, \quad \phi_{i}=t_{i}+\xi_{i}, \quad \bar{\phi}_{i}=t_{i}-\xi_{i}, \quad \mathcal{T}^{(i)}=\mathcal{T}\left(T^{(i)}, \sigma\right), \quad i=1 \ldots n
\end{aligned}
$$

in sector $\sigma$ of each open-string WZW orbifold. The explicit formulae for the inverse inertia tensor $\mathcal{L}$ and the twisted representation matrices $\mathcal{T}$ are given in Eqs. $(2.2 \mathrm{c}, \mathrm{d})$, while 
the integers $\bar{n}(r), \rho(\sigma)$ are defined in the $H$-eigenvalue problem (2.3a) of the underlying untwisted theory.

The twisted open-string KZ system in Eq. (4.35) is another central result of this paper. For brevity we will not give the $\partial_{t_{i}}, \partial_{\xi_{i}}$ form of this system, which is easily obtained by linear combination.

As an explicit example, we give the information needed to realize the twisted $\mathrm{KZ}$ systems of the open-string WZW permutation orbifolds:

$$
\begin{gathered}
n(r) \mu \rightarrow \hat{j} a j, \quad \frac{\bar{n}(r)}{\rho(\sigma)}=\frac{\overline{\hat{j}}}{f_{j}(\sigma)}, \quad g=\oplus_{I} \mathfrak{g}^{I}, \quad \mathfrak{g}^{I} \simeq \text { simple } \mathfrak{g} \\
\mathcal{L}_{\hat{\mathfrak{g}}(\sigma)}^{n(r) \mu ; n(s) \nu}(\sigma) \rightarrow \mathcal{L}_{\hat{\mathfrak{g}}(\sigma)}^{\hat{j} a j ; \hat{l} b l}(\sigma)=\delta^{j l} \frac{1}{f_{j}(\sigma)} \frac{\eta^{a b}}{2 k+Q_{\mathfrak{g}}} \\
\mathcal{T}_{n(r) \mu}(T, \sigma) \rightarrow \mathcal{T}_{\hat{j} a j}(T, \sigma)=T_{a} t_{\hat{j} j}(\sigma) \\
\overline{\hat{j}}=0, \ldots, f_{j}(\sigma)-1, \quad a=1, \ldots \operatorname{dim} \mathfrak{g} .
\end{gathered}
$$

This class of open strings arises when we appropriate our initial data from the sectors of any closed-string WZW permutation orbifold [6, 7, 9] on semisimple $g$. The cycle notation in Eq. (4.36) and the branes of these permutation-twisted open WZW strings were discussed in Subsec. 3.5.

Similarly, the explicit data [6, 7, 9, 10] for the various closed-string orbifolds on simple $g$ can be substituted into Eq. (4.35) to obtain the twisted KZ systems of the corresponding open-string WZW orbifolds.

\subsection{The One-Sided Form of the Twisted KZ Systems}

In the two-sided notation above, the twisted representation matrices $\mathcal{T}$ act on both sides of the open-string primary fields $\hat{g}$ and the open-string correlators $\hat{A}_{\sigma}$. The open-string dynamics is however more transparent when expressed in an equivalent one-sided notation [23, 16]:

$$
\begin{gathered}
\hat{\hat{g}}(\mathcal{T}, \bar{z}, z, \sigma)^{N(s) \nu ; N(r) \mu} \equiv \hat{g}(\mathcal{T}, \bar{z}, z, \sigma)_{N(r) \mu}{ }^{N(s) \nu} \\
(B \hat{g}(\mathcal{T}, \bar{z}, z, \sigma) C)_{N(r) \mu}{ }^{N(s) \nu}=B_{N(r) \mu}{ }^{N(t) \delta} \hat{g}_{N(t) \delta}^{N(u) \epsilon} C_{N(u) \epsilon}{ }^{N(s) \nu} \\
=-\tilde{\hat{g}}^{N(u) \epsilon ; N(t) \delta} C_{N(u) \epsilon}{ }^{N(s) \nu}(\bar{B})_{N(t) \delta} \delta^{N(r) \mu} \\
=-(\tilde{\hat{g}}(\mathcal{T}, \bar{z}, z, \sigma) C \otimes \bar{B})^{N(s) \nu ; N(r) \mu} \\
\bar{B}=-B^{t}, \quad\left(B^{t}\right)_{N(r) \mu}^{N(s) \nu} \equiv B_{N(s) \nu}^{N(r) \mu} .
\end{gathered}
$$


Here $t$ is matrix transpose and $B^{t}$ is the image on the right of $B$ on the left. In our application, we will then need the barred ${ }^{\ddagger 6}$ matrices $\overline{\mathcal{T}}$

$$
\begin{aligned}
\overline{\mathcal{T}}_{n(r) \mu}(T, \sigma) & \equiv-\mathcal{T}_{n(r) \mu}(T, \sigma)^{t} \\
& =\chi(\sigma)_{n(r) \mu} U(\sigma)_{n(r) \mu}{ }^{a} U(\bar{T}, \sigma) \bar{T}_{a} U^{\dagger}(\bar{T}, \sigma)=\mathcal{T}_{n(r) \mu}(\bar{T}, \sigma) \\
{\left[\overline{\mathcal{T}}_{n(r) \mu},\right.} & \left.\overline{\mathcal{T}}_{n(r) \mu}\right]=i \mathcal{F}_{n(r) \mu ; n(s) \nu}{ }^{n(r)+n(s), \delta}(\sigma) \overline{\mathcal{T}}_{n(r)+n(s), \delta}
\end{aligned}
$$

which are the image of $\mathcal{T}$ and moreover satisfy the same orbifold Lie algebra (2.2f). We remind the reader that, as shown in Eqs. (4.37b), (4.37c), $\overline{\mathcal{T}}$ always acts on the right indices of $\tilde{\hat{g}}$, while $\mathcal{T}$ acts on the left indices. This $C \otimes \bar{B}$ bookkeeping should be born in mind even though we sometimes neglect the ordering in the tensor product

$$
\overline{\mathcal{T}} \otimes \mathcal{T} \simeq \mathcal{T} \otimes \overline{\mathcal{T}}
$$

for notational convenience.

It is straightforward to reexpress all the results above in the one-sided notation, but we limit ourselves here to the one-sided form of the twisted open-string KZ system

$$
\begin{gathered}
\tilde{\hat{A}}_{\sigma}(\mathcal{T}, \xi, t) \equiv{ }_{\sigma}\left\langle 0\left|\tilde{\hat{g}}\left(\mathcal{T}^{(1)}, \xi_{1}, t_{1}\right) \ldots \tilde{\hat{g}}\left(\mathcal{T}^{(n)}, \xi_{n}, t_{n}\right)\right| 0\right\rangle_{\sigma} \\
\frac{1}{2} \partial_{+i} \tilde{\hat{A}}_{\sigma}(\mathcal{T}, \xi, t)=\tilde{\hat{A}}_{\sigma}(\mathcal{T}, \xi, t) \hat{W}_{i}(\xi, t, \sigma), \quad \frac{1}{2} \partial_{-i} \tilde{\hat{A}}_{\sigma}(\mathcal{T}, \xi, t)=\tilde{\hat{A}}_{\sigma}(\mathcal{T}, \xi, t) \hat{\bar{W}}_{i}(\xi, t, \sigma) \\
\hat{W}_{i}(\xi, t, \sigma)=2 i \mathcal{L}_{\hat{\mathfrak{g}}(\sigma)}^{n(r) \mu ;-n(r), \nu}(\sigma)\left(\sum_{j \neq i} \frac{e^{i \frac{\bar{n}(r)}{\rho(\sigma)}\left(\phi_{j}-\phi_{i}\right)}}{1-e^{i\left(\phi_{j}-\phi_{i}\right)}} \mathcal{T}_{n(r) \mu}^{(j)}+\sum_{j} \frac{e^{i \frac{\bar{n}(r)}{\rho(\sigma)}\left(\bar{\phi}_{j}-\phi_{i}\right)}}{1-e^{i\left(\bar{\phi}_{j}-\phi_{i}\right)}} \overline{\mathcal{T}}_{n(r) \mu}^{(j)}\right. \\
\left.-\frac{\bar{n}(r)}{\rho(\sigma)} \mathcal{T}_{n(r) \mu}^{(i)}\right) \mathcal{T}_{-n(r), \nu}^{(i)}+i \mathcal{D}_{\hat{\mathfrak{g}}(\sigma)}\left(\mathcal{T}^{(i)}\right) \\
\hat{\bar{W}}_{i}(\xi, t, \sigma)=2 i \mathcal{L}_{\hat{\mathfrak{g}}(\sigma)}^{n(r) \mu ;-n(r), \nu}(\sigma)\left(\sum_{j \neq i} \frac{e^{i \frac{\bar{n}(r)}{\rho(\sigma)}\left(\bar{\phi}_{j}-\bar{\phi}_{i}\right)}}{1-e^{i\left(\bar{\phi}_{j}-\bar{\phi}_{i}\right)}} \overline{\mathcal{T}}_{n(r) \mu}^{(j)}+\sum_{j} \frac{e^{i \frac{\bar{n}(r)}{\rho(\sigma)}\left(\phi_{j}-\bar{\phi}_{i}\right)}}{1-e^{i\left(\phi_{j}-\bar{\phi}_{i}\right)}} \mathcal{T}_{n(r) \mu}^{(j)}\right. \\
\left.-\frac{\bar{n}(r)}{\rho(\sigma)} \overline{\mathcal{T}}_{n(r) \mu}^{(i)}\right) \overline{\mathcal{T}}_{-n(r), \nu}^{(i)}+i \mathcal{D}_{\hat{\mathfrak{g}}(\sigma)}\left(\overline{\mathcal{T}}^{(i)}\right) \\
\tilde{\hat{A}}_{\sigma}(\mathcal{T}, \xi, t) \sum_{i=1}^{n}\left(\mathcal{T}_{0, \mu}^{(i)} \otimes \mathbb{1}+\mathbb{1}_{\hat{\mathfrak{g}}(\sigma)} \otimes \overline{\mathcal{T}}_{0, \mu}^{(i)}\right)=0 \\
\mathcal{L}_{\hat{\mathcal{T}})=}^{n(r) \mu ;-n(r), \nu} \overline{\mathcal{T}}_{n(r) \mu} \overline{\mathcal{T}}_{-n(r), \nu}=\mathcal{D}_{\hat{\mathfrak{g}}(\sigma)}(\mathcal{T})^{t}=\mathcal{D}_{\hat{\mathfrak{g}}(\sigma)(\mathcal{T})}
\end{gathered}
$$

\footnotetext{
${ }^{\ddagger 6}$ The last forms of $\overline{\mathcal{T}}$ in 4.38a use the eigenvector matrix relation $U(\bar{T}, \sigma)=U(T, \sigma)^{*}$ chosen for untwisted rep $\bar{T}$ in Ref. [16].
} 
which holds in sector $\sigma$ of $A_{g}^{\text {open }}(H) / H$. Here $\hat{W}, \hat{\bar{W}}$ are the twisted KZ connections of the system. In the $\frac{\bar{n}(r)}{\rho(\sigma)} \overline{\mathcal{T}} \overline{\mathcal{T}}$ term of $\hat{\bar{W}}$, we have used an $n(r) \mu \leftrightarrow-n(r), \nu$ exchange to order the twisted representation matrices as shown. We remind that, in spite of the numerical equality (4.40f), the twisted conformal weight matrix $\mathcal{D}_{\hat{\mathfrak{g}}(\sigma)}\left(\overline{\mathcal{T}}^{(i)}\right)$ operates on the right indices of $\tilde{\hat{g}}\left(\mathcal{T}^{(i)}\right)$.

A more familiar form of the twisted open-string $\mathrm{KZ}$ system is obtained in terms of the reduced correlators $\tilde{\hat{F}}_{\sigma}$

$$
\begin{aligned}
& \tilde{\hat{F}}_{\sigma}(\mathcal{T}, \bar{z}, z) \equiv \prod_{i=1}^{n} \tilde{\hat{A}}_{\sigma}(\mathcal{T}, \xi, t) z_{i}^{-\mathcal{D}_{\hat{\mathfrak{g}}(\sigma)}\left(\mathcal{T}^{(i)}\right)} \otimes \bar{z}_{i}^{-\mathcal{D}_{\hat{\mathfrak{g}}(\sigma)}\left(\overline{\mathcal{T}}^{(i)}\right)} \\
& \partial_{i} \tilde{\hat{F}}_{\sigma}(\mathcal{T}, \bar{z}, z)=\tilde{\hat{F}}_{\sigma}(\mathcal{T}, \bar{z}, z) \hat{W}_{i}(\bar{z}, z, \sigma), \quad \bar{\partial}_{i} \tilde{\hat{F}}_{\sigma}(\mathcal{T}, \bar{z}, z)=\tilde{\hat{F}}_{\sigma}(\mathcal{T}, \bar{z}, z) \hat{\bar{W}}_{i}(\bar{z}, z, \sigma) \\
& \hat{W}_{i}(\bar{z}, z, \sigma)=2 \mathcal{L}_{\hat{\mathfrak{g}}(\sigma)}^{n(r) \mu ;-n(r), \nu}(\sigma)\left(\sum_{j \neq i}\left(\frac{z_{j}}{z_{i}}\right)^{\frac{\bar{n}(r)}{\rho(\sigma)}} \frac{\mathcal{T}_{n(r) \mu}^{(j)} \otimes \mathcal{T}_{-n(r), \nu}^{(i)}}{z_{i}-z_{j}}\right. \\
& \left.+\sum_{j}\left(\frac{\bar{z}_{j}}{z_{i}}\right)^{\frac{\bar{n}(r)}{\rho(\sigma)}} \frac{\overline{\mathcal{T}}_{n(r) \mu}^{(j)} \otimes \mathcal{T}_{-n(r), \nu}^{(i)}}{z_{i}-\bar{z}_{j}}-\frac{\bar{n}(r)}{\rho(\sigma)} \frac{1}{z_{i}} \mathcal{T}_{n(r) \mu}^{(i)} \mathcal{T}_{-n(r), \nu}^{(i)}\right) \\
& \hat{\bar{W}}_{i}(\bar{z}, z, \sigma)=2 \mathcal{L}_{\hat{\mathfrak{g}}(\sigma)}^{n(r) \mu ;-n(r), \nu}(\sigma)\left(\sum_{j \neq i}\left(\frac{\bar{z}_{j}}{\bar{z}_{i}}\right)^{\frac{\bar{n}(r)}{\rho(\sigma)}} \frac{\overline{\mathcal{T}}_{n(r) \mu}^{(j)} \otimes \overline{\mathcal{T}}_{-n(r), \nu}^{(i)}}{\bar{z}_{i}-\bar{z}_{j}}\right. \\
& \left.+\sum_{j}\left(\frac{z_{j}}{\bar{z}_{i}}\right)^{\frac{\bar{n}(r)}{\rho(\sigma)}} \frac{\mathcal{T}_{n(r) \mu}^{(j)} \otimes \overline{\mathcal{T}}_{-n(r), \nu}^{(i)}}{\bar{z}_{i}-z_{j}}-\frac{\bar{n}(r)}{\rho(\sigma)} \frac{1}{\bar{z}_{i}} \overline{\mathcal{T}}_{n(r) \mu}^{(i)} \overline{\mathcal{T}}_{-n(r), \nu}^{(i)}\right) \\
& \tilde{\hat{F}}_{\sigma}(\mathcal{T}, \bar{z}, z) \sum_{i=1}^{n}\left(\mathcal{T}_{0, \mu}^{(i)} \otimes \mathbb{1}+\mathbb{1} \otimes \overline{\mathcal{T}}_{0, \mu}^{(i)}\right)=0 \\
& z_{i} \equiv e^{i \phi_{i}}, \quad \bar{z}_{i} \equiv e^{i \bar{\phi}_{i}}, \quad \partial_{i} \equiv \frac{\partial}{\partial z_{i}}=-\frac{i}{2 z_{i}} \partial_{+i}, \quad \bar{\partial}_{i} \equiv \frac{\partial}{\partial \bar{z}_{i}}=-\frac{i}{2 \bar{z}_{i}} \partial_{-i} \\
& \left(\frac{z_{j}}{z_{i}}\right)^{\frac{\bar{n}(r)}{\rho(\sigma)}} \equiv e^{i \frac{\bar{n}(r)}{\rho(\sigma)}\left(\phi_{j}-\phi_{i}\right)}, \quad\left(\frac{\bar{z}_{j}}{z_{i}}\right)^{\frac{\bar{n}(r)}{\rho(\sigma)}} \equiv e^{i \frac{\bar{n}(r)}{\rho(\sigma)}\left(\bar{\phi}_{j}-\phi_{i}\right)} \\
& \left(\frac{z_{j}}{\bar{z}_{i}}\right)^{\frac{\bar{n}(r)}{\rho(\sigma)}} \equiv e^{i \frac{\bar{n}(r)}{\rho(\sigma)}\left(\phi_{j}-\bar{\phi}_{i}\right)}, \quad\left(\frac{\bar{z}_{j}}{\bar{z}_{i}}\right)^{\frac{\bar{n}(r)}{\rho(\sigma)}} \equiv e^{i \frac{\bar{n}(r)}{\rho(\sigma)}\left(\bar{\phi}_{j}-\bar{\phi}_{i}\right)} \\
& \sigma=0, \ldots, N_{c}-1
\end{aligned}
$$

where the variables $\bar{z}, z$ are the Minkowski-space analogues of the usual Euclidean variables on the sphere. An analytic continuation of this system to Euclidean space would presumably maintain the phase conventions in Eqs. $(4.41 \mathrm{~g}, \mathrm{~h})$. 
As emphasized for the untwisted case in Ref. [23] and expected generally in open strings, the system (4.41) shows interactions among charges $\mathcal{T}^{(i)}$ at $z_{i}$ and image charges $\overline{\mathcal{T}}^{(i)}$ at $\bar{z}_{i}$.

In fact, the twisted open-string KZ system (4.41) can be understood as a "doubled" but ordinary chiral orbifold KZ system [6, 17, 9, 10, on $2 n$ variables:

$$
\begin{aligned}
& \hat{F}_{\sigma}(\mathcal{T},\{z\}) \equiv \tilde{\hat{F}}_{\sigma}(\mathcal{T}, \bar{z}, z), \quad \partial_{\kappa} \hat{F}_{\sigma}(\mathcal{T},\{z\})=\hat{F}_{\sigma}(\mathcal{T},\{z\}) \hat{W}_{\kappa}(\mathcal{T},\{z\}, \sigma) \\
& \hat{W}_{\kappa}(\mathcal{T},\{z\}, \sigma)=2 \mathcal{L}_{\hat{\mathfrak{g}}(\sigma)}^{n(r) \mu ;-n(r), \nu}(\sigma)\left[\sum_{\rho \neq \kappa}\left(\frac{z_{\rho}}{z_{\kappa}}\right)^{\frac{n(r)}{\rho(\sigma)}} \frac{1}{z_{\kappa \rho}} \mathcal{T}_{n(r) \mu}^{(\rho)} \otimes \mathcal{T}_{-n(r), \nu}^{(\kappa)}\right. \\
& \left.-\frac{1}{z_{\kappa}} \frac{n(r)}{\rho(\sigma)} \mathcal{T}_{n(r) \mu}^{(\kappa)} \mathcal{T}_{-n(r), \nu}^{(\kappa)}\right] \\
& \hat{F}_{\sigma}(\mathcal{T},\{z\}) \sum_{\kappa=1}^{2 n} \mathcal{T}_{0, \mu}^{(\kappa)}=0, \quad \forall \mu \\
& \partial_{\kappa} \equiv \frac{\partial}{\partial z_{\kappa}}, \quad z_{\kappa \rho} \equiv z_{\kappa}-z_{\rho}, \quad z_{\kappa} \equiv \begin{cases}z_{\kappa}, & \kappa=1 \ldots n, \\
\bar{z}_{\kappa-n}, & \kappa=n+1 \ldots 2 n\end{cases} \\
& \mathcal{T}_{n(r) \mu}^{(\kappa)} \equiv \begin{cases}\mathcal{T}_{n(r) \mu}\left(T^{(\kappa)}, \sigma\right) & \kappa=1 \ldots n \\
\mathcal{T}_{n(r) \mu}\left(\bar{T}^{(\kappa-n)}, \sigma\right) & \kappa=n+1 \ldots 2 n .\end{cases}
\end{aligned}
$$

It follows that the $n$-point correlators of our twisted open WZW strings have the same general structure as the $2 n$-point correlators of closed-string orbifold theory. This structure for the correlators of open-string CFTs 44, 45] was emphasized in the untwisted open WZW theory of Ref. 23] and in the open-string sectors of the WZW orientation orbifolds [16].

\section{Discussion}

In this paper, we have constructed what we call the basic class of twisted open WZW strings. This class consists of all the sectors $\sigma=0 \ldots N_{c}-1$ of the general open-string

orbifold $A_{g}^{\text {open }}(H) / H$, which is an orbifold of the Giusto-Halpern open string $A_{g}^{\text {open }}(H)$ by a symmetry $H \subset \operatorname{Aut}(g)$.

Our construction generalizes the untwisted construction of Ref. [23, and all of our results reduce in the untwisted sector $\sigma=0$ to the corresponding results of that reference, including e. g. the untwisted open-string $\mathrm{KZ}$ equations

$$
\begin{gathered}
\tilde{F}(\bar{z}, z) \equiv \prod_{i=1}^{n} \tilde{A}(T, \xi, t) z_{i}^{-\Delta\left(T^{(i)}\right)} \otimes \bar{z}_{i}^{-\Delta\left(T^{(i)}\right)} \\
\partial_{i} \tilde{F}(\bar{z}, z)=\tilde{F}(\bar{z}, z) W_{i}(\bar{z}, z), \quad \bar{\partial}_{i} \tilde{F}(\bar{z}, z)=\tilde{F}(\bar{z}, z) \bar{W}_{i}(\bar{z}, z)
\end{gathered}
$$




$$
\begin{gathered}
W_{i}(\bar{z}, z)=2 L_{g}^{a b}\left(\sum_{j \neq i} \frac{T_{a}^{(j)} \otimes T_{b}^{(i)}}{z_{i}-z_{j}}+\sum_{j} \frac{\bar{T}_{a}^{(j)} \otimes T_{b}^{(i)}}{z_{i}-\bar{z}_{j}}\right) \\
\bar{W}_{i}(\bar{z}, z)=2 L_{g}^{a b}\left(\sum_{j \neq i} \frac{\bar{T}_{a}^{(j)} \otimes \bar{T}_{b}^{(i)}}{\bar{z}_{i}-\bar{z}_{j}}+\sum_{j} \frac{T_{a}^{(j)} \otimes \bar{T}_{b}^{(i)}}{\bar{z}_{i}-z_{j}}\right) \\
\tilde{F}(\bar{z}, z) \sum_{i=1}^{n}\left(T_{a}^{(i)} \otimes \mathbb{1}+\mathbb{1} \otimes \bar{T}_{a}^{(i)}\right)=0, \quad \forall a=1 \ldots \operatorname{dim} g \\
{\left[T_{a}, T_{b}\right]=i f_{a b}{ }^{c} T_{c}, \quad\left[\bar{T}_{a}, \bar{T}_{b}\right]=i f_{a b}{ }^{c} \bar{T}_{c}}
\end{gathered}
$$

where $\mathrm{T}$ is any irrep of $\mathrm{g}$ and $\bar{T}=-T^{t}$. We remind the reader however that these correlators must be $H$-symmetrized because they now reside in sector $\sigma=0$ of an (open-string) orbifold.

As noted in the introduction, there is another known class of twisted open WZW strings, namely the open-string sectors of the WZW orientation orbifolds [16, 17, and it is natural to ask whether these twisted open strings are included among the constructions in our basic class. Certainly one does not expect the basic class to contain all the open-string orientation-orbifold sectors, because the sectors of each $A_{g}^{\text {open }}(H) / H$ are labelled by a complete set of conjugacy classes of $H$, whereas this is not always true for the open-string sectors of an orientation orbifold. In fact, we can be certain that the basic class contains no open-string orientation-orbifold sectors for the following technical reason: The twisted representation matrices associated to the left- and right-movers of $A_{g}^{\text {open }}(H) / H$ are $\mathcal{T}$ and $\overline{\mathcal{T}}=-\mathcal{T}^{t}$ respectively, while in the orientation orbifolds the left- and right-mover twisted representation matrices are not the bar of each other.

The construction of this paper should therefore be generalized in order to include at least the open-string orientation-orbifold sectors, and presumably many other twisted open WZW strings. The general twisted boundary state equation of App. A suggests the appropriate generalization: The idea is to introduce into the present construction an extra automorphism $\tilde{\omega}(n(r), \sigma)$ of the twisted current algebra - which is expected to generate new twisted open WZW strings T-dual to our basic class at $\tilde{\omega}=1$. It is of course well-known even at the untwisted level that N-N and D-D strings are T-dual to each other, and are related by just such an automorphism. Moreover, it is not difficult to check that the left- and right-mover twisted representation matrices of the open-string WZW orientation-orbifold sectors are indeed related by an automorphism of the twisted current algebra. With these clues, we will return to discuss the open-string picture of the general twisted open WZW string in a future paper. 


\section{Acknowledgements}

For helpful discussions, we thank O. Ganor, J. Gomis, S. Giusto, P. Hořava, N. Ishibashi and C. Schweigert.

This work was supported in part by the Director, Office of Energy Research, Office of High Energy and Nuclear Physics, Division of High Energy Physics of the U.S. Department of Energy under Contract DE-AC03-76SF00098 and in part by the National Science Foundation under grant PHY00-98840.

\section{A General Twisted Boundary States}

The development of the text followed the standard open-string picture [23] of open strings, in which the strings are described by a single set of current modes and a corresponding single Virasoro algebra. On the other hand, in the standard closed-string picture of open strings (see e. g. Refs. [46, 47]), one describes the open string by a boundary state equation in the space of left- and right-mover current modes.

In this appendix, we will discuss and extend what is known about the boundary states in the closed-string picture of twisted open WZW strings. In this picture, we begin with the twisted left- and right-mover current algebras [6] in sector $\sigma$ of any WZW orbifold $A_{g}(H) / H$

$$
\begin{aligned}
& {\left[\hat{J}_{n(r) \mu}\left(m+\frac{n(r)}{\rho(\sigma)}\right), \hat{J}_{n(s) \nu}\left(n+\frac{n(s)}{\rho(\sigma)}\right)\right]=} \\
& i \mathcal{F}_{n(r) \mu ; n(s) \nu}^{n(r)+n(s), \delta}(\sigma) \hat{J}_{n(r)+n(s), \delta}\left(m+n+\frac{n(r)+n(s)}{\rho(\sigma)}\right) \\
& +\left(m+\frac{n(r)}{\rho(\sigma)}\right) \delta_{m+n+\frac{n(r)+n(s)}{\rho(\sigma)}, 0} \mathcal{G}_{n(r) \mu ;-n(r) \nu}(\sigma) \\
& {\left[\hat{\bar{J}}_{n(r) \mu}\left(m+\frac{n(r)}{\rho(\sigma)}\right), \hat{\bar{J}}_{n(s) \nu}\left(n+\frac{n(s)}{\rho(\sigma)}\right)\right]=} \\
& i \mathcal{F}_{n(r) \mu ; n(s) \nu}^{n(r)+n(s), \delta}(\sigma) \hat{\bar{J}}_{n(r)+n(s), \delta}\left(m+n+\frac{n(r)+n(s)}{\rho(\sigma)}\right) \\
& -\left(m+\frac{n(r)}{\rho(\sigma)}\right) \delta_{m+n+\frac{n(r)+n(s)}{\rho(\sigma)}, 0} \mathcal{G}_{n(r) \mu ;-n(r) \nu}(\sigma) \\
& {\left[\hat{J}_{n(r) \mu}\left(m+\frac{n(r)}{\rho(\sigma)}\right), \hat{\bar{J}}_{n(s) \nu}\left(n+\frac{n(s)}{\rho(\sigma)}\right)\right]=0, \quad \sigma=0, \ldots, N_{c}-1} \\
& \hat{J}_{n(r) \pm \rho(\sigma), \mu}\left(m+\frac{n(r) \pm \rho(\sigma)}{\rho(\sigma)}\right)=\hat{J}_{n(r) \mu}\left(m \pm 1+\frac{n(r)}{\rho(\sigma)}\right) \\
& \hat{\bar{J}}_{n(r) \pm \rho(\sigma), \mu}\left(m+\frac{n(r) \pm \rho(\sigma)}{\rho(\sigma)}\right)=\hat{\bar{J}}_{n(r) \mu}\left(m \pm 1+\frac{n(r)}{\rho(\sigma)}\right)
\end{aligned}
$$


where $N_{c}$ is the number of conjugacy classes of $H \subset A u t(g)$. The left-mover modes $\hat{J}$ are the same as those of the text, and $\mathcal{G}(\sigma)$ and $\mathcal{F}(\sigma)$ are the twisted metric and twisted structure constants of the sector.

Because of the sign reversal of the central term ${ }^{\ddagger 7}$ in the right-mover current algebra (A.1b), Ref. [6] was able to give the following consistent equation for the twisted openstring boundary states of twisted sector $\sigma$

$$
\begin{aligned}
& \left(\hat{J}_{n(r) \mu}\left(m+\frac{n(r)}{\rho(\sigma)}\right)+\hat{\bar{J}}_{n(r) \mu}\left(m+\frac{n(r)}{\rho(\sigma)}\right)\right)|B\rangle_{\sigma}=0, \quad \sigma=0, \ldots, N_{c}-1 \\
& {\left[(\hat{J}+\hat{\bar{J}})_{n(r) \mu}\left(m+\frac{n(r)}{\rho(\sigma)}\right),(\hat{J}+\hat{\bar{J}})_{n(s) \nu}\left(n+\frac{n(s)}{\rho(\sigma)}\right)\right]=} \\
& \quad=i \mathcal{F}_{n(r) \mu ; n(s) \nu} n(r)+n(s), \delta(\sigma)(\hat{J}+\hat{\bar{J}})_{n(r)+n(s), \delta}\left(m+n+\frac{n(r)+n(s)}{\rho(\sigma)}\right)
\end{aligned}
$$

without any consideration of the rectification problem [6, 7, 9, 10, reviewed in App. B. We expect that these twisted boundary states describe the twisted open strings in the basic class of this paper, but we emphasize that for $\sigma \neq 0$ no attempt has thus far been made to find explicit solutions of this system.

As an extension, we consider next the corresponding action of the Virasoro generators on this set of twisted boundary states. For this computation, we will need the explicit forms [6] of the left- and right-mover Virasoro generators in terms of twisted current modes:

$$
\begin{aligned}
L_{\sigma}(m)= & \mathcal{L}_{\hat{\mathfrak{g}}(\sigma)}^{n(r)-n(r), \nu}(\sigma) \sum_{p \in \mathbb{Z}}: \hat{J}_{n(r) \mu}\left(p+\frac{n(r)}{\rho(\sigma)}\right) \hat{J}_{-n(r), \nu}\left(m-p-\frac{n(r)}{\rho(\sigma)}\right): \\
= & \mathcal{L}_{\hat{\mathfrak{g}}(\sigma)}^{n(r) \mu ;-n(r), \nu}(\sigma)\left(\sum_{p \in \mathbb{Z}}: \hat{J}_{n(r) \mu}\left(p+\frac{n(r)}{\rho(\sigma)}\right) \hat{J}_{-n(r), \nu}\left(m-p-\frac{n(r)}{\rho(\sigma)}\right):_{M}\right. \\
& \left.-i \frac{\bar{n}(r)}{\rho(\sigma)} \mathcal{F}_{n(r) \mu ;-n(r), \nu} 0, \delta(\sigma) \hat{J}_{0, \delta}(m)\right)+\delta_{m, 0} \hat{\Delta}_{0}(\sigma) \\
\bar{L}_{\sigma}(m)= & \mathcal{L}_{\hat{\mathfrak{g}}(\sigma) \mu ;-n(r), \nu}^{n(\sigma)} \sum_{p \in \mathbb{Z}}: \hat{\bar{J}}_{n(r) \mu}\left(p+\frac{n(r)}{\rho(\sigma)}\right) \hat{\bar{J}}_{-n(r), \nu}\left(-m-p-\frac{n(r)}{\rho(\sigma)}\right): \\
= & \mathcal{L}_{\hat{\mathfrak{g}}(\sigma)}^{n(r) \mu ;-n(r), \nu}(\sigma)\left(\sum_{p \in \mathbb{Z}}: \hat{\bar{J}}_{n(r) \mu}\left(p+\frac{n(r)}{\rho(\sigma)}\right) \hat{\bar{J}}_{-n(r), \nu}\left(-m-p-\frac{n(r)}{\rho(\sigma)}\right):_{\bar{M}}\right. \\
& \left.-i \frac{\overline{-n(r)}}{\rho(\sigma)} \mathcal{F}_{n(r) \mu ;-n(r), \nu} 0, \delta(\sigma) \hat{\bar{J}}_{0, \delta}(-m)\right)+\delta_{m, 0} \hat{\Delta}_{0}(\sigma)
\end{aligned}
$$

\footnotetext{
${ }^{\ddagger 7}$ This surprising feature follows directly from the WZW orbifold action, and has also been explicitly checked at the level of characters [6].
} 


$$
\begin{aligned}
& : \hat{J}_{n(r) \mu}\left(m+\frac{n(r)}{\rho(\sigma)}\right) \hat{J}_{n(s) \nu}\left(n+\frac{n(s)}{\rho(\sigma)}\right):_{M} \equiv \theta\left(m+\frac{n(r)}{\rho(\sigma)} \geq 0\right) \hat{J}_{n(s) \nu}\left(n+\frac{n(s)}{\rho(\sigma)}\right) \hat{J}_{n(r) \mu}\left(m+\frac{n(r)}{\rho(\sigma)}\right) \\
& +\theta\left(m+\frac{n(r)}{\rho(\sigma)}<0\right) \hat{J}_{n(r) \mu}\left(m+\frac{n(r)}{\rho(\sigma)}\right) \hat{J}_{n(s) \nu}\left(n+\frac{n(s)}{\rho(\sigma)}\right) \\
& : \hat{\bar{J}}_{n(r) \mu}\left(m+\frac{n(r)}{\rho(\sigma)}\right) \hat{\bar{J}}_{n(s) \nu}\left(n+\frac{n(s)}{\rho(\sigma)}\right):_{\bar{M}} \equiv \theta\left(m+\frac{n(r)}{\rho(\sigma)} \leq 0\right) \hat{\bar{J}}_{n(s) \nu}\left(n+\frac{n(s)}{\rho(\sigma)}\right) \hat{\bar{J}}_{n(r) \mu}\left(m+\frac{n(r)}{\rho(\sigma)}\right) \\
& +\theta\left(m+\frac{n(r)}{\rho(\sigma)}>0\right) \hat{\bar{J}}_{n(r) \mu}\left(m+\frac{n(r)}{\rho(\sigma)}\right) \hat{\bar{J}}_{n(s) \nu}\left(n+\frac{n(s)}{\rho(\sigma)}\right) \\
& \hat{\Delta}_{0}(\sigma) \equiv \sum_{r, \mu, \nu} \mathcal{L}_{\hat{\mathfrak{g}}(\sigma) \mu ;-n(r), \nu}^{n(\sigma)}(\sigma) \frac{\bar{n}(r)}{2 \rho(\sigma)}\left(1-\frac{\bar{n}(r)}{\rho(\sigma)}\right) \mathcal{G}_{n(r) \mu ;-n(r), \nu}(\sigma) \\
& \frac{\overline{-n(r)}}{\rho(\sigma)}= \begin{cases}1-\frac{\bar{n}(r)}{\rho(\sigma)} & \text { for } \bar{n}(r) \neq 0 \\
0 & \text { for } \bar{n}(r)=0\end{cases} \\
& {\left[L_{\sigma}(m), L_{\sigma}(n)\right]=(m-n) L_{\sigma}(m+n)+\delta_{m+n, 0} \frac{c_{g}}{12} m\left(m^{2}-1\right)} \\
& {\left[\bar{L}_{\sigma}(m), \bar{L}_{\sigma}(n)\right]=(m-n) \bar{L}_{\sigma}(m+n)+\delta_{m+n, 0} \frac{c_{g}}{12} m\left(m^{2}-1\right)} \\
& {\left[L_{\sigma}(m), \bar{L}_{\sigma}(n)\right]=0 \text {. }}
\end{aligned}
$$

Here $: \cdot:_{M}$ and $: \cdot:_{\bar{M}}$ are the standard mode normal orderings of the orbifold program [3, 5, 6], and : · : is the mode form of operator-product normal ordering. The left-mover Virasoro generators $L_{\sigma}(m)$ are the same as those of the text (see e. g. Eq. (2.1a)), and $\hat{\Delta}_{0}(\sigma)$ in Eq. A.3e is the conformal weight of the scalar twist field.

We are interested in particular in the relation of $\bar{L}_{\sigma}|B\rangle_{\sigma}$ to $L_{\sigma}|B\rangle_{\sigma}$, as implied by the boundary-state equation (A.2). To study $\bar{L}_{\sigma}|B\rangle_{\sigma}$ we found the following identities useful

$$
\begin{gathered}
: \hat{\bar{J}}_{n(r) \mu}\left(m+\frac{n(r)}{\rho(\sigma)}\right) \hat{\bar{J}}_{-n(r), \nu}\left(n-\frac{n(r)}{\rho(\sigma)}\right): \bar{M}|B\rangle_{\sigma}= \\
\left(: \hat{J}_{n(r) \mu}\left(m+\frac{n(r)}{\rho(\sigma)}\right) \hat{J}_{-n(r), \nu}\left(n-\frac{n(r)}{\rho(\sigma)}\right):_{M}+i \delta_{m+\frac{n(r)}{\rho(\sigma)}, 0} \mathcal{F}_{0, \mu ; 0, \nu} 0, \delta(\sigma) \hat{J}_{-n(r), \delta}\left(n-\frac{n(r)}{\rho(\sigma)}\right)\right)|B\rangle_{\sigma} \\
\mathcal{L}_{\hat{\mathfrak{g}}(\sigma)}^{0, \mu ; 0, \nu}(\sigma) \mathcal{F}_{0, \mu ; 0, \nu}{ }^{0, \delta}(\sigma)=\mathcal{L}_{\hat{\mathfrak{g}}(\sigma)}^{n(r) \mu ;-n(r), \nu}(\sigma) \mathcal{F}_{n(r) \mu ;-n(r), \nu}{ }^{0, \delta}(\sigma)=0 \\
\mathcal{L}_{\hat{\mathfrak{g}}(\sigma)}^{n(r) \mu ;-n(r), \nu}(\sigma) \frac{\overline{-n(r)}}{\rho(\sigma)} \mathcal{F}_{n(r) \mu ;-n(r), \nu}{ }^{0, \delta}(\sigma) \hat{\bar{J}}_{0, \delta}(m)|B\rangle_{\sigma} \\
=\mathcal{L}_{\hat{\mathfrak{g}}(\sigma)}^{n(r) \mu ;-n(r), \nu}(\sigma) \frac{\bar{n}(r)}{\rho(\sigma)} \mathcal{F}_{n(r) \mu ;-n(r), \nu}{ }^{0, \delta}(\sigma) \hat{J}_{0, \delta}(m)|B\rangle_{\sigma}
\end{gathered}
$$

where A.4a follows from the boundary-state equation, the mode-ordering definitions (A.3c,d) and the twisted current algebra. The relation in Eq. (A.4b) is obtained from the symmetries $(2.2 \mathrm{~b}, \mathrm{c})$ of $\mathcal{L}$ and $\mathcal{F}$, and Eq. (A.4c) then follows from Eq. (A.3f) and the boundary-state equation. 
With the identities in Eq. (A.4) and the Virasoro generators in Eq. (A.3), we then verify the twisted open-string Virasoro conditions on the twisted boundary states:

$$
\begin{gathered}
\left(L_{\sigma}(m)-\bar{L}_{\sigma}(-m)\right)|B\rangle_{\sigma}=0, \quad \sigma=0, \ldots, N_{c}-1 \\
{\left[L_{\sigma}(m)-\bar{L}_{\sigma}(-m), L_{\sigma}(n)-\bar{L}_{\sigma}(-n)\right]=(m-n)\left(L_{\sigma}(m+n)-\bar{L}_{\sigma}(-m-n)\right) .}
\end{gathered}
$$

Such Virasoro conditions are familiar [46] in the closed string picture of untwisted open WZW strings.

The boundary state equation in Eq. A.2 is in fact a special case of the following general twisted boundary-state equation

$$
\begin{gathered}
\left(\hat{J}_{n(r) \mu}\left(m+\frac{n(r)}{\rho(\sigma)}\right)+\tilde{\omega}(n(r), \sigma)_{\mu}{ }^{\nu} \hat{\bar{J}}_{n(r), \nu}\left(m+\frac{n(r)}{\rho(\sigma)}\right)\right)|B\rangle_{\sigma}=0 \\
\sigma=0, \ldots, N_{c}-1
\end{gathered}
$$

which is consistent when $\tilde{\omega}$ is any mode-number-preserving automorphism of the right- or left-mover twisted mode algebra (A.1):

$$
\begin{gathered}
\tilde{\omega}(n(r), \sigma)_{\mu}{ }^{\kappa} \tilde{\omega}(n(s), \sigma)_{\nu}{ }^{\lambda} \mathcal{F}_{n(r), \kappa ; n(s), \lambda}{ }^{n(t), \epsilon}(\sigma)=\mathcal{F}_{n(r) \mu ; n(s) \nu}{ }^{n(t) \delta}(\sigma) \tilde{\omega}(n(t), \sigma)_{\delta}{ }^{\epsilon} \\
\tilde{\omega}(n(r), \sigma)_{\mu}{ }^{\kappa} \tilde{\omega}(n(s), \sigma)_{\nu}{ }^{\lambda} \mathcal{G}_{n(r) \sigma=0, \ldots, N_{c}-1, \kappa ; n(s), \lambda}(\sigma)=\mathcal{G}_{n(r) \mu ; n(s) \nu}(\sigma) \\
\mathcal{G}^{n(r), \kappa ; n(s), \lambda}(\sigma) \tilde{\omega}(n(r), \sigma)_{\kappa}{ }^{\mu} \tilde{\omega}(n(s), \sigma)_{\lambda}{ }^{\nu}=\mathcal{G}^{n(r) \mu ; n(s) \nu}(\sigma) .
\end{gathered}
$$

We expect that such an $\tilde{\omega}$ modification can also be used to describe the general twisted open WZW string in the open-string picture of the text (see the discussion in Sec. 5).

Similarly, the Virasoro conditions

$$
\left(L_{\sigma}(m)-\bar{L}_{\sigma}(-m)\right)|B\rangle_{\sigma}=0, \quad \sigma=0, \ldots, N_{c}-1
$$

hold as well for the general boundary state in Eq. (A.6). To see this, a helpful identity is

$$
\mathcal{L}_{\hat{\mathfrak{g}}(\sigma)}^{n(r), \kappa ; n(s), \lambda}(\sigma) \tilde{\omega}(n(r), \sigma)_{\kappa}{ }^{\mu} \tilde{\omega}(n(s), \sigma)_{\lambda}{ }^{\nu}=\mathcal{L}_{\hat{\mathfrak{g}}(\sigma)}^{n(r) \mu ; n(s) \nu}(\sigma)
$$

which follows from Eqs. (2.2C) and (A.7c) when the underlying untwisted current algebra has the special form:

$$
\begin{gathered}
g=\oplus_{I} \mathfrak{g}^{I}, \quad \mathfrak{g}^{I} \simeq \operatorname{simple} g, \quad k^{I}=k \\
\Rightarrow \mathcal{L}_{\hat{\mathfrak{g}}(\sigma)}^{n(r) \mu ; n(s) \nu}(\sigma)=\frac{k}{2 k+Q} \mathcal{G}^{n(r) \mu ; n(s) \nu}(\sigma) .
\end{gathered}
$$

The proof for the general case follows because the general untwisted current algebra can be considered as a direct sum of these special cases. 
The general twisted boundary-state equation in Eq. (A.6) reduces to the standard ${ }^{\ddagger 8}$ untwisted boundary state equation in sector $\sigma=0$

$$
\left(J_{a}(m)+\omega_{a}^{b} \bar{J}_{b}(-m)\right)|B\rangle_{0}=0, \quad a, b=1 \ldots \operatorname{dim} g, \quad \omega \in \operatorname{Aut}(g)
$$

where $\hat{\bar{J}}$ reduces $[\underline{6}$ to $\bar{J}(-m)$ and $\tilde{\omega} \rightarrow \omega$ is any mode-preserving automorphism of untwisted affine $g$.

\section{B Rectification in the Closed-String WZW Orbifolds}

In the text, we noticed that the equal-time current algebra of the twisted open WZW string is not isomorphic in the bulk to the twisted current algebra of the corresponding closed-string orbifold - but we claimed instead that the open-string algebra was isomorphic to the rectified current algebra of the closed-string orbifold. For application in the text, we therefore review and extend in this appendix the concept of rectification [6, 17, 9, 10] in closed-string orbifold theory, beginning with the rectified current algebra.

The left- and right-mover current algebras of ordinary closed-string orbifolds $A_{g}(H) / H$ are given in Eq. A.1 , and we remind the reader that the right-mover commutators are not a copy of the left-mover commutators due to the sign reversal of the central term 6 . In what follows we will assume that the right-mover algebra can however be rectified into a copy of the left-mover algebra

$$
\begin{aligned}
& \hat{\bar{J}}_{n(r) \mu}^{\sharp}\left(m+\frac{n(r)}{\rho(\sigma)}\right) \equiv \theta(n(r), \sigma)_{\mu}{ }^{\nu} \hat{\bar{J}}_{-n(r), \nu}\left(-m-\frac{n(r)}{\rho(\sigma)}\right) \\
& {\left[\hat{J}_{n(r) \mu}\left(m+\frac{n(r)}{\rho(\sigma)}\right), \hat{J}_{n(s) \nu}\left(n+\frac{n(s)}{\rho(\sigma)}\right)\right] }=i \mathcal{F}_{n(r) \mu ; n(s) \nu}^{n(r)+n(s), \delta}(\sigma) \hat{J}_{n(r)+n(s), \delta}\left(m+n+\frac{n(r)+n(s)}{\rho(\sigma)}\right) \\
&+\left(m+\frac{n(r)}{\rho(\sigma)}\right) \delta_{m+n+\frac{n(r)+n(s)}{\rho(\sigma)}, 0} \mathcal{G}_{n(r) \mu ;-n(r) \nu}(\sigma) \\
& {\left[\hat{\bar{J}}_{n(r) \mu}^{\sharp}\left(m+\frac{n(r)}{\rho(\sigma)}\right), \hat{\bar{J}}_{n(s) \nu}^{\sharp}\left(n+\frac{n(s)}{\rho(\sigma)}\right)\right] }=i \mathcal{F}_{n(r) \mu ; n(s) \nu}^{n(r)+n(s), \delta}(\sigma) \hat{\bar{J}}_{n(r)+n(s), \delta}^{\sharp}\left(m+n+\frac{n(r)+n(s)}{\rho(\sigma)}\right) \\
&+\left(m+\frac{n(r)}{\rho(\sigma)}\right) \delta_{m+n+\frac{n(r)+n(s)}{\rho(\sigma)}, 0} \mathcal{G}_{n(r) \mu ;-n r n}(\sigma)
\end{aligned}
$$

where $\sharp$ denotes the rectified right-mover modes. The existence of such a rectification is equivalent to the following conditions

$$
\begin{gathered}
\theta(n(r), \sigma)_{\mu}{ }^{\kappa} \theta(n(s), \sigma)_{\nu}{ }^{\lambda} \mathcal{G}_{-n(r), \kappa ;-n(s), \lambda}(\sigma)=\mathcal{G}_{n(r) \mu ; n(s) \nu}(\sigma) \\
\theta(n(r), \sigma)_{\mu}{ }^{\kappa} \theta(n(s), \sigma)_{\nu}{ }^{\lambda} \mathcal{F}_{-n(r), \kappa ;-n(s), \lambda}{ }^{-n(t), \epsilon}(\sigma)=\mathcal{F}_{n(r) \mu ; n(s) \nu}{ }^{n(t) \delta}(\sigma) \theta(n(t), \sigma)_{\delta}{ }^{\epsilon}
\end{gathered}
$$

\footnotetext{
${ }^{\ddagger 8}$ See for example Refs. 46, 47.
} 
on the twisted metric and twisted structure constants of sector $\sigma$.

Finding such a rectification $\{\theta\}$ for a given twisted right-mover current algebra is nontrivial, but this rectification problem has in fact been solved on a case-by-case basis

- the WZW permutation orbifolds [6, 7, 9]

- the inner-automorphic WZW orbifolds 6] on simple $\mathfrak{g}$

- the outer-automorphic WZW orbifolds [7, 10] on simple $\mathfrak{g}$

for all basic orbifold types. Given these results, one might expect that all twisted rightmover current algebras can be rectified. We emphasize however that this has not yet been systematically discussed for more general twisted current algebras, such as the doublytwisted current algebras of Refs. [2, 4] - which arise from automorphisms that are compositions of the basic types above.

In the application of the text, we will need the equal-time form of the rectified current algebra. Towards this, we first use the mode algebra (B.1) to obtain the equal-time algebra of the unrectified orbifold currents on the cylinder [11]:

$$
\begin{aligned}
& \hat{J}_{n(r) \mu}(\xi, t) \equiv \sum_{m \in \mathbb{Z}} \hat{J}_{n(r) \mu}\left(m+\frac{n(r)}{\rho(\sigma)}\right) e^{-i\left(m+\frac{n(r)}{\rho(\sigma)}\right)(t+\xi)}=\hat{J}_{n(r) \pm \rho(\sigma), \mu}(\xi, t) \\
& \hat{\bar{J}}_{n(r) \mu}(\xi, t) \equiv \sum_{m \in \mathbb{Z}} \hat{\bar{J}}_{n(r) \mu}\left(m+\frac{n(r)}{\rho(\sigma)}\right) e^{i\left(m+\frac{n(r)}{\rho(\sigma)}\right)(t-\xi)}=\hat{\bar{J}}_{n(r) \pm \rho(\sigma), \mu}(\xi, t) \\
& \hat{J}_{n(r) \mu}(\xi+2 \pi, \sigma)=e^{-2 \pi i \frac{n(r)}{\rho(\sigma)}} \hat{J}_{n(r) \mu}(\xi, \sigma), \quad \hat{\bar{J}}_{n(r) \mu}(\xi+2 \pi, \sigma)=e^{-2 \pi i \frac{n(r)}{\rho(\sigma)}} \hat{\bar{J}}_{n(r) \mu}(\xi, \sigma) \\
& {\left[\hat{J}_{n(r) \mu}(\xi, t, \sigma), \hat{J}_{n(s) \nu}(\eta, t, \sigma)\right]=2 \pi i\left(\mathcal{F}_{n(r) \mu ; n(s) \nu}{ }^{n(r)+n(s), \delta}(\sigma) \hat{J}_{n(r)+n(s), \delta}(\eta, t, \sigma)\right.} \\
& \left.+\delta_{n(r)+n(s), 0 \bmod \rho(\sigma)} \mathcal{G}_{n(r) \mu ;-n(r) \nu}(\sigma) \partial_{\xi}\right) \delta_{\frac{n(r)}{\rho(\sigma)}}(\xi-\eta) \\
& {\left[\hat{\bar{J}}_{n(r) \mu}(\xi, t, \sigma), \hat{\bar{J}}_{n(s) \nu}(\eta, t, \sigma)\right]=2 \pi i\left(\mathcal{F}_{n(r) \mu ; n(s) \nu} n(r)+n(s), \delta(\sigma) \hat{\bar{J}}_{n(r)+n(s), \delta}(\eta, t, \sigma)\right.} \\
& \left.-\delta_{n(r)+n(s), 0 \bmod \rho(\sigma)} \mathcal{G}_{n(r) \mu ;-n(r) \nu}(\sigma) \partial_{\xi}\right) \delta_{\frac{n(r)}{\rho(\sigma)}}(\xi-\eta) \\
& {\left[\hat{J}_{n(r) \mu}(\xi, t, \sigma), \hat{\bar{J}}_{n(s) \nu}(\eta, t, \sigma)\right]=0, \quad \sigma=0, \ldots, N_{c}-1}
\end{aligned}
$$


Except for the range of $\xi$, the twisted left-mover current $\hat{J}$ here is the same current called $\hat{J}^{(+)}$in Eq. (2.5), and the phase-modified Dirac delta function $\delta_{n(r) / \rho(\sigma)}(\xi-\eta)$ is that given in Eq. (2.8).

The rectified right-mover twisted currents are then defined ${ }^{\ddagger 9}$ in terms of the rectified modes (B.1a) as follows:

$$
\begin{gathered}
\hat{\bar{J}}_{n(r) \mu}^{\sharp}(\xi, t, \sigma) \equiv \sum_{m \in \mathbb{Z}} \hat{\bar{J}}_{n(r) \mu}^{\sharp}\left(m+\frac{n(r)}{\rho(\sigma)}\right) e^{-i\left(m+\frac{n(r)}{\rho(\sigma)}\right)(t-\xi)}=\theta(n(r), \sigma)_{\mu}{ }^{\nu} \hat{\bar{J}}_{-n(r), \nu}(\xi, t, \sigma) \\
\hat{\bar{J}}_{n(r) \mu}^{\sharp}(\xi+2 \pi, t, \sigma)=e^{2 \pi i \frac{n(r)}{\rho(\sigma)}} \hat{\bar{J}}_{n(r) \mu}^{\sharp}(\xi, t, \sigma) .
\end{gathered}
$$

Then the rectified equal-time current algebra

$$
\begin{aligned}
{\left[\hat{J}_{n(r) \mu}(\xi, t, \sigma), \hat{J}_{n(s) \nu}(\eta, t, \sigma)\right] } & =2 \pi i\left(\mathcal{F}_{n(r) \mu ; n(s) \nu} n(r)+n(s), \delta(\sigma) \hat{J}_{n(r)+n(s), \delta}(\eta, t, \sigma)\right. \\
& \left.+\delta_{n(r)+n(s), 0 \bmod \rho(\sigma)} \mathcal{G}_{n(r) \mu ;-n(r) \nu}(\sigma) \partial_{\xi}\right) \delta_{\frac{n(r)}{\rho(\sigma)}}(\xi-\eta) \\
{\left[\hat{\bar{J}}_{n(r) \mu}^{\sharp}(\xi, t, \sigma), \hat{\bar{J}}_{n(s) \nu}^{\sharp}(\eta, t, \sigma)\right] } & =2 \pi i\left(\mathcal{F}_{n(r) \mu ; n(s) \nu} n(r)+n(s), \delta(\sigma) \hat{\bar{J}}_{n(r)+n(s), \delta}^{\sharp}(\eta, t, \sigma)\right. \\
& \left.-\delta_{n(r)+n(s), 0 \bmod \rho(\sigma)} \mathcal{G}_{n(r) \mu ;-n(r) \nu}(\sigma) \partial_{\xi}\right) \delta_{-\frac{n(r)}{\rho(\sigma)}}(\xi-\eta) \\
{\left[\hat{J}_{n(r) \mu}(\xi, t, \sigma), \hat{\bar{J}}_{n(s) \nu}^{\sharp}(\eta, t, \sigma)\right] } & =0, \quad \sigma=0, \ldots, N_{c}-1
\end{aligned}
$$

is obtained from the unrectified algebra (B.4). The only difference between this rectified algebra and Eq. (B.4) is the phase of the $\delta$-function in Eq. (B.6b).

We will also need to consider the orbifold stress tensors on the cylinder

$$
\begin{gathered}
\hat{T}_{\sigma}(\xi, t) \equiv \frac{1}{2 \pi} \sum_{m \in \mathbb{Z}} L_{\sigma}(m) e^{-i m(t+\xi)}=\frac{1}{2 \pi} \mathcal{L}_{\hat{\mathfrak{g}}(\sigma)}^{n(r) \mu ;-n(r), \nu}(\sigma): \hat{J}_{n(r) \mu}(\xi, t) \hat{J}_{-n(r), \nu}(\xi, t): \\
\hat{\bar{T}}_{\sigma}(\xi, t) \equiv \frac{1}{2 \pi} \sum_{m \in \mathbb{Z}} \bar{L}_{\sigma}(m) e^{i m(t-\xi)}=\frac{1}{2 \pi} \mathcal{L}_{\hat{\mathfrak{g}}(\sigma)}^{n(r) \mu ;-n(r), \nu}(\sigma): \hat{\bar{J}}_{n(r) \mu}(\xi, t) \hat{\bar{J}}_{-n(r), \nu}(\xi, t): \\
\hat{T}_{\sigma}(\xi+2 \pi, t)=\hat{T}_{\sigma}(\xi, t), \quad \hat{\bar{T}}_{\sigma}(\xi+2 \pi, t)=\hat{\bar{T}}_{\sigma}(\xi, t)
\end{gathered}
$$

\footnotetext{
${ }^{\ddagger 9}$ The definition (B.5a) is the cylinder analogue of the rectified right-mover twisted currents on the sphere given in Ref. [6].
} 
where the normal ordering : · : is defined by the mode form of operator-product normal ordering (see Eqs. (2.1a) and (A.3) $)$. Then we find the following equal-time operator algebra

$$
\begin{gathered}
{\left[\hat{T}_{\sigma}(\xi, t), \hat{T}_{\sigma}(\eta, t)\right]=i\left(\left(\hat{T}_{\sigma}(\xi, t)+\hat{T}_{\sigma}(\eta, t)\right)-\frac{c_{g}}{24 \pi}\left(\partial_{\xi}^{2}+1\right)\right) \partial_{\xi} \delta(\xi-\eta)} \\
{\left[\hat{\bar{T}}_{\sigma}(\xi, t), \hat{\bar{T}}_{\sigma}(\eta, t)\right]=-i\left(\left(\hat{\bar{T}}_{\sigma}(\xi, t)+\hat{\bar{T}}_{\sigma}(\eta, t)\right)-\frac{c_{g}}{24 \pi}\left(\partial_{\xi}^{2}+1\right)\right) \partial_{\xi} \delta(\xi-\eta)} \\
{\left[\hat{T}_{\sigma}(\xi, t), \hat{\bar{T}}_{\sigma}(\eta, t)\right]=0} \\
c_{g} \equiv 2 \mathcal{G}_{n(r) \mu ; n(s) \nu}(\sigma) \mathcal{L}_{\hat{\mathfrak{g}}(\sigma)}^{n(r) \mu ; n(s) \nu}(\sigma)=2 G^{a b} L_{g}^{a b}=\sum_{I} \frac{2 k_{I} \operatorname{dim} \mathfrak{g}^{I}}{2 k_{I}+Q_{I}} \\
{\left[\hat{T}_{\sigma}(\xi, t), \hat{J}_{n(r) \mu}(\eta, t, \sigma)\right]=-i \partial_{\eta}\left(\hat{J}_{n(r) \mu}(\eta, t, \sigma) \delta(\xi-\eta)\right)} \\
{\left[\hat{\bar{T}}_{\sigma}(\xi, t), \hat{\bar{J}}_{n(r) \mu}^{\sharp}(\eta, t, \sigma)\right]=i \partial_{\eta}\left(\hat{\bar{J}}_{n(r) \mu}^{\sharp}(\eta, t, \sigma) \delta(\xi-\eta)\right)} \\
{\left[\hat{T}_{\sigma}(\xi, t), \hat{\bar{J}}_{n(r) \mu}^{\sharp}(\eta, t, \sigma)\right]=\left[\hat{\bar{T}}_{\sigma}(\xi, t), \hat{J}_{n(r) \mu}(\eta, t, \sigma)\right]=0}
\end{gathered}
$$

which, except for the commutators with the rectified currents $\hat{\bar{J}}^{\sharp}$, was given earlier in Ref. [11]. We also mention that the right-mover stress tensor can be rewritten in terms of $\hat{\bar{J}} \sharp$ :

$$
\hat{\bar{T}}_{\sigma}(\xi, t)=\frac{1}{2 \pi} \mathcal{L}_{\hat{\mathfrak{g}}(\sigma)}^{n(r) \mu ; n(s) \nu}(\sigma): \hat{\bar{J}}_{n(r) \mu}^{\sharp}(\xi, t, \sigma) \hat{\bar{J}}_{n(s) \nu}^{\sharp}(\xi, t, \sigma): .
$$

The argument for (B.10) follows the same line as that given at the end of App. A, using now the relations

$$
\begin{aligned}
\mathcal{G}^{n(r) \mu ; n(s) \nu}(\sigma) \theta(n(r), \sigma)_{\mu}{ }^{\kappa} \theta(n(s), \sigma)_{\nu}{ }^{\lambda}=\mathcal{G}^{-n(r), \kappa ;-n(s), \lambda}(\sigma) \\
\Longrightarrow \mathcal{L}_{\hat{\mathfrak{g}}(\sigma) \mu ; n(s) \nu}^{n(r)}(\sigma) \theta(n(r), \sigma)_{\mu}{ }^{\kappa} \theta(n(s), \sigma)_{\nu}{ }^{\lambda}=\mathcal{L}_{\hat{\mathfrak{g}}(\sigma)}^{-n(r) \kappa ;-n(s) \lambda}(\sigma)
\end{aligned}
$$

which follow from Eqs. (B.2a) and (A.9).

For classical closed-string WZW orbifolds, we list the following rectified relations

$$
\begin{gathered}
\hat{T}_{\sigma}(\xi, t)=\frac{1}{4 \pi} \mathcal{G}^{n(r) \mu ; n(s) \nu}(\sigma) \hat{J}_{n(r) \mu}(\xi, t, \sigma) \hat{J}_{n(s) \nu}(\xi, t, \sigma) \\
\hat{\bar{T}}_{\sigma}(\xi, t)=\frac{1}{4 \pi} \mathcal{G}^{n(r) \mu ; n(s) \nu}(\sigma) \hat{\bar{J}}_{n(r) \mu}^{\sharp}(\xi, t, \sigma) \hat{\bar{J}}_{n(s) \nu}^{\sharp}(\xi, t, \sigma) \\
\hat{J}_{n(r) \mu}(\xi)=2 \pi \hat{e}^{-1}(\xi)_{n(r) \mu}{ }^{n(s) \nu} \hat{p}_{n(s) \nu}(\hat{B}, \xi)+\frac{1}{2} \partial_{\xi} \hat{x}^{n(s) \nu}(\xi) \hat{e}(\xi)_{n(s) \nu}{ }^{n(t) \delta} \mathcal{G}_{n(t) \delta ; n(r) \mu}(\sigma) \\
\hat{\bar{J}}_{n(r) \mu}^{\sharp}(\xi)=2 \pi \hat{e}^{\wedge}-1(\xi)_{n(r) \mu}^{n(s) \nu} \hat{p}_{n(s) \nu}(\hat{B}, \xi)-\frac{1}{2} \partial_{\xi} \hat{x}^{n(s) \nu}(\xi) \hat{e}^{\sharp}(\xi)_{n(s) \nu}{ }^{n(t) \delta} \mathcal{G}_{n(t) \delta ; n(r) \mu}(\sigma) \\
\hat{\bar{e}}_{n(r) \mu}(\mathcal{T}, \xi)=-i \hat{g}(\mathcal{T}, \xi) \hat{\partial}_{n(r) \mu} \hat{g}^{-1}(\mathcal{T}, \xi)=\hat{e}(\xi)_{n(r) \mu}^{n(s) \nu} \mathcal{T}_{n(s) \nu}=\hat{e}^{\sharp}(\xi)_{n(r) \mu}{ }^{n(s) \nu} \mathcal{T}_{n(s) \nu}^{\sharp}
\end{gathered}
$$




$$
\begin{gathered}
\left\{\hat{J}_{n(r) \mu}(\xi, t, \sigma), \hat{x}_{\sigma}^{n(s) \nu}(\eta, t)\right\}=-2 \pi i \hat{e}^{-1}(\eta, t, \sigma)_{n(r) \mu}{ }^{n(s) \nu} \delta_{\frac{n(r)}{\rho(\sigma)}}(\xi-\eta) \\
\left\{\hat{\bar{J}}_{n(r) \mu}^{\sharp}(\xi, t, \sigma), \hat{x}_{\sigma}^{n(s) \nu}(\eta, t)\right\}=-2 \pi i \hat{e}^{\sharp}-1(\eta, t, \sigma)_{n(r) \mu}{ }^{n(s) \nu} \delta_{-\frac{n(r)}{\rho(\sigma)}}(\xi-\eta) \\
\left\{\hat{J}_{n(r) \mu}(\xi, t, \sigma), \hat{g}(\mathcal{T}, \eta, t, \sigma)\right\}=2 \pi \hat{g}(\mathcal{T}, \eta) \mathcal{T}_{n(r) \mu} \delta_{\frac{n(r)}{\rho(\sigma)}}(\xi-\eta) \\
\left\{\hat{\bar{J}}_{n(r) \mu}^{\sharp}(\xi, t, \sigma), \hat{g}(\mathcal{T}, \eta, t, \sigma)\right\}=-2 \pi \mathcal{T}_{n(r) \mu}^{\sharp} \hat{g}(\mathcal{T}, \eta) \delta_{-\frac{n(r)}{\rho(\sigma)}}(\xi-\eta) \\
\left\{\hat{x}_{\sigma}^{n(r) \mu}(\xi, t), \hat{x}_{\sigma}^{n(s) \nu}(\eta, t)\right\}=0
\end{gathered}
$$

which have been selected for comparison with the analogous strip results of the text. To obtain these results, we used Eq. (B.2), the corresponding (unrectified) closed-string orbifold relations in Ref. [11, and the definitions:

$$
\begin{gathered}
\mathcal{T}_{n(r) \mu}^{\sharp} \equiv \theta(n(r), \sigma)_{\mu}{ }^{\nu} \mathcal{T}_{-n(r), \nu} \\
{\left[\mathcal{T}_{n(r) \mu}^{\sharp}, \mathcal{T}_{n(s) \nu}^{\sharp}\right]=i \mathcal{F}_{n(r) \mu ; n(s) \nu}{ }^{n(t) \delta}(\sigma) \mathcal{T}_{n(t) \delta}^{\sharp}} \\
\hat{\bar{e}}^{\sharp}(\xi)_{n(r) \mu}{ }^{n(s) \nu} \equiv \hat{\bar{e}}(\xi)_{n(r) \mu}{ }^{-n(s), \lambda} \theta^{-1}(n(s), \sigma)_{\lambda}{ }^{\nu} .
\end{gathered}
$$

Here $\hat{\bar{J}}^{\sharp}, \mathcal{T}^{\sharp}$ and $\hat{e}^{\sharp}$ are respectively the rectified right-mover currents, the rectified twisted representation matrices and the rectified right-invariant twisted vielbein on the group orbifold.

Looking back over the rectified results of this appendix, we may finally consider the following strip $\leftrightarrow$ cylinder map:

$$
\begin{gathered}
\hat{J}^{(+)} \leftrightarrow \hat{J}, \quad \hat{J}^{(-)} \leftrightarrow \hat{\bar{J}}^{\sharp} \\
\hat{e} \leftrightarrow \hat{e}, \quad \hat{\bar{e}} \leftrightarrow \hat{e}^{\sharp}, \quad \hat{g} \mathcal{T} \leftrightarrow \hat{g} \mathcal{T}, \quad \mathcal{T} \hat{g} \leftrightarrow \mathcal{T}^{\sharp} \hat{g} .
\end{gathered}
$$

One finds that under this map, the twisted open-string properties in Eqs. (2.7), (3.12), (3.16) and (3.38) of the text are locally twisted WZW, that is, they are isomorphic in the bulk to the rectified closed-string properties in Eqs. (B.6), (B.13), (B.14) and (B.15).

\section{More About the Phase-Modified Delta Functions}

The phase-modified Dirac delta functions $\delta_{n(r) / \rho(\sigma)}(\xi \pm \eta)$ were defined in Eq. (2.8), and the case $\delta_{n(r) / \rho(\sigma)}(\xi-\eta)$ was studied on the cylinder in Ref. [11]. For the computations of the text, one needs further information about these delta functions on the strip. 
We begin with the strip identities

$$
\begin{aligned}
& \hat{A}_{n(r) \mu}^{( \pm)} n(s) \nu(\xi, t) \delta_{\frac{n(t)}{\rho(\sigma)}}( \pm \xi-\eta)=\hat{A}_{n(r) \mu}^{(+)} n(s) \nu(\eta, t) \delta_{\frac{n(r)-n(s)+n(t)}{\rho(\sigma)}}( \pm \xi-\eta) \\
& \hat{A}_{n(r) \mu}^{( \pm)} n(s) \nu(\xi, t) \delta_{\frac{n(t)}{\rho(\sigma)}}( \pm \xi+\eta)=\hat{A}_{n(r) \mu}^{(-)} n(s) \nu(\eta, t) \delta_{\frac{n(r)-n(s)+n(t)}{\rho(\sigma)}}( \pm \xi+\eta) \\
& \text { for any } \hat{A} \text { s.t. } \hat{A}_{n(r) \mu}^{( \pm)} n(s) \nu(-\xi, t)=\hat{A}_{n(r) \mu}^{(\mp)} n(s) \nu(\xi, t) \\
& \text { and } \hat{A}_{n(r) \mu}^{(+)} n(s) \nu(\pi, t)=e^{-2 \pi i \frac{n(r)-n(s)}{\rho(\sigma)}} \hat{A}_{n(r) \mu}^{(-)} n(s) \nu(\pi, t) \\
& 0 \leq \xi, \eta \leq \pi
\end{aligned}
$$

which apply in particular to the twisted strip currents $\hat{A}^{( \pm)}=\hat{J}^{( \pm)}$when $n(s)=0$. The $\delta(\xi-\eta)$ identities here appeared in Ref. [11] as a consequence of monodromy, which is here replaced by the strip boundary condition (C.1c).

Following Ref. [11, we note the special case of Eq. (C.1) with $n(t)=n(s)-n(r)$ :

$$
\begin{aligned}
& \hat{A}_{n(r) \mu}^{( \pm)} n(s) \nu(\xi, t) \delta_{\frac{n(s)-n(r)}{\rho(\sigma)}}( \pm \xi-\eta)=\hat{A}_{n(r) \mu}^{(+)} n(s) \nu(\eta, t) \delta( \pm \xi-\eta) \\
& \hat{A}_{n(r) \mu}^{( \pm)} n(s) \nu(\xi, t) \delta_{\frac{n(s)-n(r)}{\rho(\sigma)}}( \pm \xi+\eta)=\hat{A}_{n(r) \mu}^{(-)} n(s) \nu(\eta, t) \delta( \pm \xi+\eta) .
\end{aligned}
$$

This case leads directly to the following integral identities on the strip

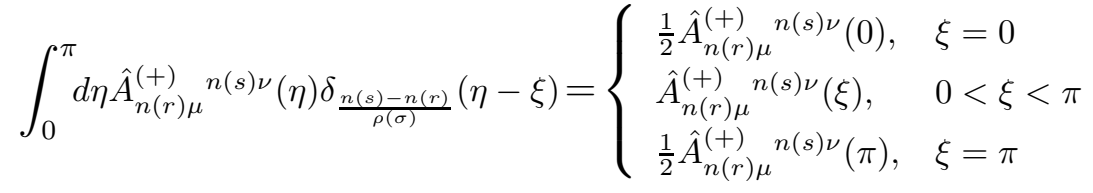

$$
\begin{aligned}
& \int_{0}^{\pi} d \eta \hat{A}_{n(r) \mu}^{(-)} n(s) \nu(\eta) \delta_{\frac{n(s)-n(r)}{\rho(\sigma)}}(-\eta+\xi)= \begin{cases}\frac{1}{2} \hat{A}_{n(r) \mu}^{(-)} n(s) \nu(0), & \xi=0 \\
\hat{A}_{n(r) \mu}^{(-)} n(s) \nu(\xi), & 0<\xi<\pi \\
\frac{1}{2} \hat{A}_{n(r) \mu}^{(-)} n(s) \nu(\pi)=\frac{1}{2} e^{2 \pi i \frac{n(r)-n(s)}{\rho(\sigma)}} \hat{A}_{n(r) \mu}^{(+)} n(s) \nu(\pi), & \xi=\pi\end{cases}
\end{aligned}
$$

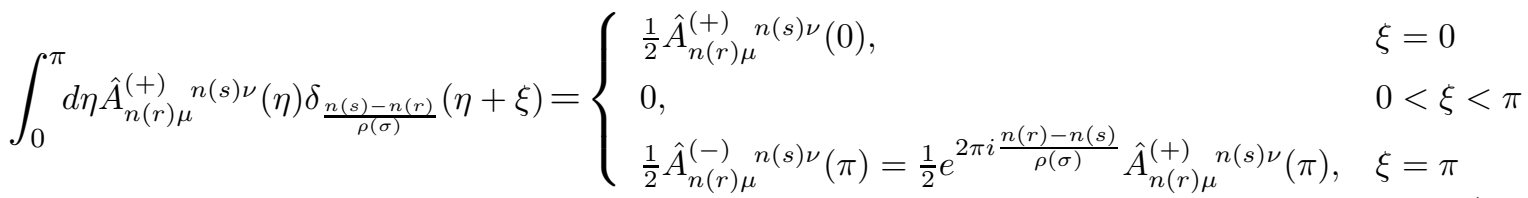

$$
\begin{aligned}
& \int_{0}^{\pi} d \eta \hat{A}_{n(r) \mu}^{(-)} n(s) \nu(\eta) \delta_{\frac{n(s)-n(r)}{\rho(\sigma)}}(-\eta-\xi)= \begin{cases}\frac{1}{2} \hat{A}_{n(r) \mu}^{(+)}{ }^{n(s) \nu}(0), & \xi=0 \\
0, & 0<\xi<\pi \\
\frac{1}{2} \hat{A}_{n(r) \mu}^{(+)} n(s) \nu(\pi), & \xi=\pi\end{cases}
\end{aligned}
$$


which generalize the integral identities given in Ref. 23] for ordinary delta functions on the strip. Comparing Eqs. (C.3a,b) with Ref. [23], one confirms that the same results are obtained with or without the phase modification of $\delta(\eta-\xi)$. This is easily understood because the phase of $\delta_{n(r) / \rho(\sigma)}$ is non-trivial only when the argument of the delta function is $2 \pi$, which is not achieved in the integrals (C.3a,b):

$$
\delta_{\frac{n(r)}{\rho(\sigma)}}(\xi-\eta) \equiv \delta(\xi-\eta) \text { when } 0 \leq \xi, \eta \leq \pi .
$$

It is not clear however that the phase-modifications can always be ignored in spatial derivatives of $\delta_{n(r) / \rho(\sigma)}(\xi-\eta)$.

A simple consequence of Eq. (C.3) is the identity

$$
\int_{0}^{\pi} d \eta\left(\hat{A}_{n(r) \mu}^{( \pm)} n(s) \nu(\eta) \delta_{\frac{n(s)-n(r)}{\rho(\sigma)}}( \pm \eta \mp \xi)+(\eta \leftrightarrow-\eta)\right)=\hat{A}_{n(r) \mu}^{( \pm)} n(s) \nu(\xi)
$$

and identities similar to Eqs. (C.1)-C.5 are easily derived for twisted fields with any number of indices. For example, the corresponding identity on $\hat{A}_{n(r) \mu ; n(s) \nu}$

$$
\int_{0}^{\pi} d \eta\left(\hat{A}_{n(r) \mu ; n(s) \nu}^{( \pm)}(\eta) \delta_{\frac{-n(s)-n(r)}{\rho(\sigma)}}( \pm \eta \mp \xi)+(\eta \leftrightarrow-\eta)\right)=\hat{A}_{n(r) \mu ; n(s) \nu}^{( \pm)}(\xi)
$$

is obtained from Eq. (C.5) by replacing $n(s) \rightarrow-n(s)$ in the phase-modified delta functions.

\section{Examples of the New Non-Commutative Geometries}

In this appendix, we consider some simple examples of the new non-commutative geometries associated to the $\{x, x\}$ brackets of the open WZW strings.

Because no non-abelian examples were worked out in Ref. [23, we begin with two examples in the case of untwisted open WZW strings $A_{g}^{\text {open }}$. For these examples we will need the following explicit forms of the adjoint action $\Omega(x)$ and the vielbein $e(x)$

$$
\begin{gathered}
\Omega(x)=g^{-1}\left(T^{a d j}, x\right)=e^{-i Y(x)}, \quad e(x)=\left(\frac{e^{i Y(x)}-1}{i Y(x)}\right) \\
Y(x) \equiv x^{i} e_{i}^{a}(0) T_{a}^{a d j}=x^{a} T_{a}^{a d j}, \quad a=1, \ldots, \operatorname{dim} g
\end{gathered}
$$

which hold for any group manifold. Here $g$ is the group element, $T^{\text {adj }}$ is the matrix adjoint rep of Lie $g$ and we have chosen $e(0)=1$ so that the Einstein-space indices are equivalent to the tangent-space indices $a \simeq i$.

Example 1: $A_{\mathfrak{s u}(2)}^{\text {open }}$ 
For $g=\mathfrak{s u}(2)$, we choose root length 1 and the standard Cartesian basis

$$
\begin{gathered}
\left(T_{a}^{a d j}\right)_{b c}=i\left(R_{a}\right)_{b c}=-i \epsilon_{a b c}, \quad \epsilon_{123}=1, \quad x^{a} T_{a}^{a d j}=i \vec{x} \cdot \vec{R}, \quad a, b, c \in\{1,2,3\} \\
(\vec{x} \cdot \vec{R})^{2 m+1}=\left(-|\vec{x}|^{2}\right)^{m}(\vec{x} \cdot \vec{R}), \quad(\vec{x} \cdot \vec{R})^{2 m+2}=\left(-|\vec{x}|^{2}\right)^{m}(\vec{x} \cdot \vec{R})^{2}, \quad m \geq 0
\end{gathered}
$$

which allows us to evaluate the following geometric quantities:

$$
\begin{gathered}
G_{a b}=k \delta_{a b}, \quad g(T, \xi)=e^{i \vec{x}(\xi) \cdot T} \\
\Omega(\xi)=\mathbb{1}+\frac{\sin |\vec{x}(\xi)|}{|\vec{x}(\xi)|} \vec{x}(\xi) \cdot \vec{R}+\frac{1-\cos |\vec{x}(\xi)|}{|\vec{x}(\xi)|^{2}}(\vec{x}(\xi) \cdot \vec{R})^{2}, \quad e(0)_{i}{ }^{a}=\delta_{i}{ }^{a} \\
G_{i j}(x(\xi))=k\left(\delta_{i j}+\frac{2 \cos |\vec{x}(\xi)|+|\vec{x}(\xi)|^{2}-2}{|\vec{x}(\xi)|^{4}}\left((\vec{x}(\xi) \cdot \vec{R})^{2}\right)_{i j}\right) .
\end{gathered}
$$

Except for the range $0 \leq \xi \leq \pi$, these forms hold as well for the corresponding closedstring WZW model $A_{\mathfrak{s u}(2)}$. Then the non-commutative geometry of the Giusto-Halpern open string $A_{\mathfrak{s u}(2)}^{o p e n}$

$$
\begin{gathered}
\left\{x^{i}(\xi, t), x^{j}(\eta, t)\right\}=i \pi\left\{\begin{array}{cc}
-\Psi^{i j}(0,0) & \text { if } \xi=\eta=0 \\
\Psi^{i j}(\pi, \pi) & \text { if } \xi=\eta=\pi \\
0 & \text { otherwise }
\end{array}\right. \\
\Psi^{i j}(\xi, \xi)=-\frac{|\vec{x}(\xi)| \sin |\vec{x}(\xi)|}{k(1-\cos |\vec{x}(\xi)|)}(\vec{x}(\xi) \cdot \vec{R})_{i j}
\end{gathered}
$$

follows directly from the results in Ref. 23] or Eq. (3.39).

Example 2: $A_{\mathfrak{s u}(2) \oplus \mathfrak{s u}(2)}^{\text {open }}$

We next consider the untwisted open WZW string on $g=\mathfrak{s u}(2) \oplus \mathfrak{s u}(2)$, whose $\mathbb{Z}_{2}$ permutation symmetry will be modded out to obtain our third (twisted) example below.

The required geometric quantities for this open WZW string

$$
\begin{gathered}
G_{a I ; b J}=k \delta_{I J} \delta_{a b}, \quad I, J=0,1, \quad a, b, c=1,2,3 \\
g(T, \xi)=\exp \left[i\left(\begin{array}{cc}
x^{0 a}(\xi) T_{a}^{(0)} & 0 \\
0 & x^{1 a}(\xi) T_{a}^{(1)}
\end{array}\right)\right], \quad T^{(0)} \simeq T^{(1)} \simeq T \\
\Omega(\xi)_{a I}{ }^{b J}=\delta_{I}{ }^{J}\left(\delta_{a}{ }^{b}+\frac{\sin \left|\vec{x}^{I}(\xi)\right|}{\left|\vec{x}^{I}(\xi)\right|}\left(\vec{x}^{I}(\xi) \cdot \vec{R}\right)_{a b}+\frac{1-\cos \left|\vec{x}^{I}(\xi)\right|}{\left|\vec{x}^{I}(\xi)\right|^{2}}\left(\left(\vec{x}^{I}(\xi) \cdot \vec{R}\right)^{2}\right)_{a b}\right) \\
G_{i I ; j J}(x(\xi))=k \delta_{I J}\left(\delta_{i j}+\frac{2 \cos \left|\vec{x}^{I}(\xi)\right|+\left|\vec{x}^{I}(\xi)\right|^{2}-2}{\left|\vec{x}^{I}(\xi)\right|^{4}}\left(\left(\vec{x}^{I}(\xi) \cdot \vec{R}\right)^{2}\right)_{i j}\right) \\
x(\xi)=\left\{x^{i I}(\xi)\right\}, i=1,2,3, I=0,1, \quad \vec{x}^{I}(\xi) \cdot \vec{R} \equiv x^{a I}(\xi) R_{a}, \quad e(0)_{i I}{ }^{a K}=\delta_{i}{ }^{a} \delta_{I}{ }^{K}
\end{gathered}
$$


are easily read off as two copies of those given in the previous example. Correspondingly, the non-commutative geometry of $A_{\mathfrak{s u}(2) \oplus \mathfrak{s u}(2)}^{\text {open }}$

$$
\begin{gathered}
\left\{x^{i I}(\xi, t), x^{j J}(\eta, t)\right\}=i \pi\left\{\begin{array}{cc}
-\Psi^{i I ; j J}(0,0) & \text { if } \xi=\eta=0 \\
\Psi^{i I ; j J}(\pi, \pi) & \text { if } \xi=\eta=\pi \\
0 & \text { otherwise }
\end{array}\right. \\
\Psi^{i I ; j J}(\xi, \xi)=-\delta^{I J} \frac{\left|\vec{x}^{I}(\xi)\right| \sin \left|\vec{x}^{I}(\xi)\right|}{k\left(1-\cos \left|\vec{x}^{I}(\xi)\right|\right)}\left(\vec{x}^{I}(\xi) \cdot \vec{R}\right)_{i j}
\end{gathered}
$$

decomposes, as expected, into two non-interacting copies of the geometry of $A_{\mathfrak{s u}(2)}^{\text {open }}$.

Example 3: $A_{s u(2) \oplus s u(2)}^{\text {open }}(H) / H, \quad H=\mathbb{Z}_{2}($ perm $)$.

As our last example, we discuss the twisted non-commutative geometry of the twisted sector of the open-string orbifold $A_{s u(2) \oplus s u(2)}^{\text {open }}\left(\mathbb{Z}_{2}\right) / \mathbb{Z}_{2}$, where the $\mathbb{Z}_{2}$ is the permutation symmetry which exchanges the two copies of $\mathfrak{s u}(2)$.

Following the development of this paper, we begin with the data of the single twisted left-mover sector $(\sigma=1)$ of the closed-string $\mathbb{Z}_{2}$ permutation orbifold $A_{s u(2) \oplus s u(2)}\left(\mathbb{Z}_{2}\right) / \mathbb{Z}_{2}$. This data includes for example the simplest orbifold affine algebra [1]

$$
\begin{gathered}
{\left[\hat{J}_{\hat{j} a}\left(m+\frac{\hat{j}}{2}\right), \hat{J}_{\hat{l} b}\left(n+\frac{\hat{l}}{2}\right)\right]=i \epsilon_{a b c} \hat{J}_{\hat{j}+\hat{l}, c}\left(m+n+\frac{\hat{j}+\hat{l}}{2}\right)+2 k \delta_{a b}\left(m+\frac{\hat{j}}{2}\right) \delta_{m+n+\frac{\hat{j}+\hat{l}}{2}, 0}} \\
\bar{n}(r) \rightarrow \overline{\hat{j}} \in\{0,1\}, \quad \mu \rightarrow a=1,2,3
\end{gathered}
$$

which provides the current-algebraic input (2.1b) for this case.

At the geometric level, the following twisted quantities

$$
\begin{aligned}
& \sigma=1: \quad \mathcal{G}_{\hat{j} a ; \hat{l} b}=2 k \delta_{a b} \delta_{\hat{j}+\hat{l}, 0 \bmod 2}, \quad \mathcal{T}_{\hat{j} a}(T)=T_{a} \tau_{\hat{j}} \\
& \hat{g}(\mathcal{T}(T), \xi)=\exp \left[i\left(\hat{x}^{0 a}(\xi) \mathbb{1}_{2}+\hat{x}^{1 a}(\xi) \tau_{1}\right) \otimes T_{a}\right], \quad \vec{\tau}=\text { Pauli matrices } \\
& \hat{\Omega}(\hat{x})=e^{-i \hat{Y}(\hat{x})}, \quad \hat{Y}(\hat{x}(\xi)) \equiv \hat{x}^{\hat{j} a}(\xi) T_{a}^{a d j} \tau_{\hat{j}}, \quad \hat{e}(\hat{x})=\left(\frac{e^{i \hat{Y}(\hat{x})}-1}{i \hat{Y}(\hat{x})}\right) \\
& \hat{\Omega}(\hat{x}(\xi))=\frac{1}{2}\left(\begin{array}{ll}
\hat{\Omega}\left(\hat{X}^{+}(\xi)\right)+\hat{\Omega}\left(\hat{X}^{-}(\xi)\right) & \hat{\Omega}\left(\hat{X}^{+}(\xi)\right)-\hat{\Omega}\left(\hat{X}^{-}(\xi)\right) \\
\hat{\Omega}\left(\hat{X}^{+}(\xi)\right)-\hat{\Omega}\left(\hat{X}^{-}(\xi)\right) & \hat{\Omega}\left(\hat{X}^{+}(\xi)\right)+\hat{\Omega}\left(\hat{X}^{-}(\xi)\right)
\end{array}\right) \\
& \hat{\Omega}\left(\hat{X}^{ \pm}(\xi)\right) \equiv \mathbb{1}+\frac{\sin \left|\hat{X}^{ \pm}(\xi)\right|}{\left|\hat{X}^{ \pm}(\xi)\right|} \hat{X}^{ \pm}(\xi) \cdot \vec{R}+\frac{1-\cos \left|\hat{X}^{ \pm}(\xi)\right|}{\left|\hat{X}^{ \pm}(\xi)\right|^{2}}\left(\hat{X}^{ \pm}(\xi) \cdot \vec{R}\right)^{2} \\
& \hat{x}^{ \pm, a}(\xi) \equiv \hat{x}^{0, a}(\xi) \pm \hat{x}^{1, a}(\xi)
\end{aligned}
$$




$$
\begin{gathered}
\hat{G}_{\hat{j} a ; \hat{l} b}(\hat{x}(\xi))=k\left(G\left(\hat{X}^{+}(\xi)\right)+(-1)^{\hat{j}+\hat{l}} G\left(\hat{X}^{-}(\xi)\right)\right)_{a b} \\
G\left(\hat{X}^{ \pm}(\xi)\right) \equiv \mathbb{1}+\frac{\left(\hat{X}^{ \pm}(\xi) \cdot \vec{R}\right)^{2}}{\left|\hat{X}^{ \pm}(\xi)\right|^{4}}\left(2 \cos \left|\hat{X}^{ \pm}(\xi)\right|+\left|\hat{X}^{ \pm}(\xi)\right|^{2}-2\right)
\end{gathered}
$$

follow from the definitions of the text. Except for the range $0 \leq \xi \leq \pi$, these are the same formulae given in Ref. [1] for the closed-string orbifold $A_{s u(2) \oplus s u(2)}\left(\mathbb{Z}_{2}\right) / \mathbb{Z}_{2}$. Then the twisted non-commutative geometry of sector $\sigma=1$ of the open-string orbifold $A_{\text {su }}^{\text {open }(2) \oplus s u(2)}\left(\mathbb{Z}_{2}\right) / \mathbb{Z}_{2}$

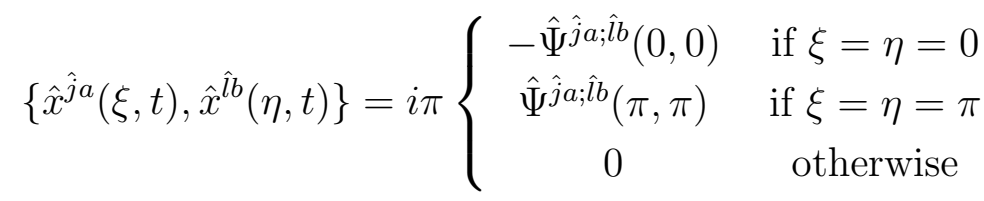

$$
\begin{aligned}
& \hat{\Psi}^{\hat{j} a ; \hat{l} b}(0,0)=-\frac{1}{4 k}\left(\frac{\left|\hat{X}^{+}(0)\right| \sin \left|\hat{X}^{+}(0)\right|}{1-\cos \left|\hat{X}^{+}(0)\right|}\left(\hat{x}^{+}(0) \cdot \vec{R}\right)_{a b}+\right. \\
& \left.+(-1)^{\hat{j}+\hat{l}} \frac{\left|\hat{X}^{-}(0)\right| \sin \left|\hat{X}^{-}(0)\right|}{1-\cos \left|\hat{X}^{-}(0)\right|}\left(\hat{X}^{-}(0) \cdot \vec{R}\right)_{a b}\right) \\
& \hat{\Psi}^{\hat{j} a ; \hat{l} b}(\pi, \pi)=-\frac{1}{4 k}\left[(-1)^{\hat{j}}\left(\left(\hat{\psi}^{+} \hat{\psi}^{-}\right)^{t}-\left(\hat{\psi}^{-} \hat{\psi}^{+}\right)\right)+(-1)^{\hat{l}}\left(\left(\hat{\psi}^{-} \hat{\psi}^{+}\right)^{t}-\left(\hat{\psi}^{+} \hat{\psi}^{-}\right)\right)\right]_{a b} \\
& \hat{\psi}^{ \pm} \equiv \mathbb{1}+\frac{1}{2}\left(\hat{X}^{ \pm}(\pi) \cdot \vec{R}\right)+\left(\frac{1}{\left|\hat{x}^{ \pm}(\pi)\right|^{2}}-\frac{\sin \left|\hat{x}^{ \pm}(\pi)\right|}{2\left|\hat{X}^{ \pm}(\pi)\right|\left(1-\cos \left|\hat{X}^{ \pm}(\pi)\right|\right)}\right)\left(\hat{x}^{ \pm}(\pi) \cdot \vec{R}\right)^{2}
\end{aligned}
$$

follows from Eqs. (D.8) and (3.38). As in the text, superscript $t$ is matrix transpose. The example above is the simplest permutation-twisted open WZW string.

\section{References}

[1] L. Borisov, M. B. Halpern, and C. Schweigert, "Systematic approach to cyclic orbifolds," Int. J. Mod. Phys. A13 (1998) 125, hep-th/9701061.

[2] J. Evslin, M. B. Halpern, and J. E. Wang, "General Virasoro construction on orbifold affine algebra," Int. J. Mod. Phys. A14 (1999) 4985, hep-th/9904105

[3] J. de Boer, J. Evslin, M. B. Halpern, and J. E. Wang, "New duality transformations in orbifold theory," Int. J. Mod. Phys. A15 (2000) 1297, hep-th/9908187.

[4] J. Evslin, M. B. Halpern, and J. E. Wang, "Cyclic coset orbifolds," Int. J. Mod. Phys. A15 (2000) 3829-3860, hep-th/9912084

[5] M. B. Halpern and J. E. Wang, "More about all current-algebraic orbifolds," Int. J. Mod. Phys. A16 (2001) 97, hep-th/0005187

[6] J. de Boer, M. B. Halpern, and N. A. Obers, "The operator algebra and twisted KZ equations of WZW orbifolds," J. High Energy Phys. 10 (2001) 011, hep-th/0105305. 
[7] M. B. Halpern and N. A. Obers, "Two Large Examples in Orbifold Theory: Abelian Orbifolds and the Charge Conjugation Orbifold on su(n)," Int. J. Mod. Phys. A17 (2002) 3897, hep-th/0203056

[8] M. B. Halpern and F. Wagner, "The General Coset Orbifold Action," Int. J. Mod. Phys. A18 (2003) 19, hep-th/0205143

[9] M. B. Halpern and C. Helfgott, "Extended Operator Algebra and Reducibility in the WZW Permutation Orbifolds," Int. J. Mod. Phys. A18 (2003) 1773, hep-th/0208087.

[10] O. Ganor, M. B. Halpern, C. Helfgott and N. A. Obers, "The Outer-Automorphic WZW Orbifolds on $\mathfrak{s o}(2 n)$, including Five Triality Orbifolds on $\mathfrak{s o}(8)$," J. High Energy Phys. 0212 (2002) 019, hep-th/0211003

[11] J. deBoer, M. B. Halpern and C. Helfgott, "Twisted Einstein Tensors and Orbifold Geometry," Int. J. Mod. Phys. A18 (2003) 3489, hep-th/0212275

[12] V. G. Kac and I. T. Todorov, "Affine orbifolds and rational conformal field theory extensions of $W_{1+\infty}, "$ Comm. Math. Phys. 190 (1997) 57, hep-th/9612078

[13] P. Bantay, "Characters and modular properties of permutation orbifolds," Phys. Lett. B419 (1998) 175-178, hep-th/9708120.

[14] L. Birke, J. Fuchs, and C. Schweigert, "Symmetry breaking boundary conditions and WZW orbifolds," Adv. Theor. Math. Phys. 3 (1999) 671-726, hep-th/9905038.

[15] V. G. Kac, R. Longo, F. Xu, "Solitons in Affine and Permutation Orbifolds," hep-th/0312512

[16] M. B. Halpern and C. Helfgott, "Twisted Open Strings from Closed Strings: The WZW Orientation Orbifolds," Int. J. Mod. Phys. A19 (2004) 2233, hep-th/0306014

[17] M. B. Halpern and C. Helfgott, "On the Target-Space Geometry of the Open-String Orientation-Orbifold Sectors," Ann.of Phys. 310 (2004) 302, hep-th/0309101.

[18] A. Sagnotti, "Open Strings and Their Symmetry Groups," ROM2F-87/25, talk presented at the Cargese Summer Institute on Non-Perturbative Methods in Field Theory, Cargese, Italy, July 16-30, 1987, hep-th/0208020

[19] P. Horava, "Strings on World Sheet Orbifolds," Nucl. Phys B327 (1989) 461.

[20] J. Dai, R. G. Leigh and J. Polchinski, "New Connections Among String Theories," Mod. Phys. Lett. A4 (1989) 2073.

[21] P. Horava, "Chern-Simons Gauge Theory on Orbifolds: Open Strings from Three Dimensions," J. Geom. Phys 21 (1996) 1, hep-th/9404101

[22] R. Dijkgraaf, E. Verlinde and H. Verlinde, "Matrix String Theory," Nucl. Phys. B 500 (1997) 43, hep-th/9703030

[23] S. Giusto and M. B. Halpern, "Hamiltonian Formulation of Open WZW Strings," Int. J. Mod. Phys. A16 (2001) 3237, hep-th/0101220

[24] M. R. Douglas and G. Moore, "D-Branes, Quivers, and ALE Instantons," hep-th/9603167

[25] C. Angelantonj and A. Sagnotti, "Open Strings," Phys. Rept. 371 (2002) 1-150, hep-th/0204089.

[26] K. Bardakci and M. B. Halpern, "New dual quark models," Phys. Rev. D3 (1971) 2493.

[27] M. B. Halpern, "The two faces of a dual pion-quark model," Phys. Rev. D4 (1971) 2398.

[28] R. Dashen and Y. Frishman, "Four fermion interactions and scale invariance," Phys. Rev. D11 (1975) 2781.

[29] V. G. Knizhnik and A. B. Zamolodchikov, "Current Algebra and Wess-Zumino Model in Two Dimensions," Nucl. Phys. B247 (1984) 83.

[30] G. Segal, unpublished.

[31] V. Kac, "Simple graded Lie algebras of finite growth," Funct. Anal. Appl. 1 (1967) 328.

[32] R. V. Moody, "Lie algebras associated with generalized Cartan matrices," Bull. Am. Math. Soc. 73 (1967) 217-221. 
[33] M. B. Halpern and C. B. Thorn, "Two faces of a dual pion-quark model. II. Fermions and other things," Phys. Rev. D4 (1971) 3084.

[34] M. B. Halpern, "Quantum 'Solitons' which are $\mathfrak{s u}(N)$ Fermions," Phys. Rev D12 (1975) 1684; "Equivalent-Boson Method and Free Currents in Two-Dimensional Gauge Theories," Phys. Rev. D13 (1976) 337.

[35] M. B. Halpern and E. Kiritsis, "General Virasoro construction on affine g," Mod. Phys. Lett. A4 (1989) 1373.

[36] M. B. Halpern, E. B. Kiritsis, and N. A. Obers, "The Lie $h$ invariant conformal field theories and the Lie $h$ invariant graphs," Int. J. Mod. Phys. A7 (Suppl. 1A) (1992) 339, hep-th/9110001

[37] M. B. Halpern, E. Kiritsis, N. A. Obers, and K. Clubok, "Irrational conformal field theory," Physics Reports 265, Nos. 1 \& 2, (1996) 1-138, hep-th/9501144

[38] J. de Boer and M. B. Halpern, "Unified Einstein-Virasoro Master Equation in the Nonlinear Sigma Model," Int. J. Mod. Phys. A12 (1997) 1551, hep-th/9606025.

[39] A. Yu. Alekseev and V. Schomerus, "D-branes in the WZW model," Phys. Rev. D60, (1999) 061901, hep-th/9812193

[40] K. Gawedzki, I. Todorov and P. Tran-Ngoc-Bich, "Canonical quantization of the boundary Wess-Zumino-Witten model," hep-th/0101170

[41] W. Siegel, "Strings with Dimension-Dependent Intercept," Nucl. Phys B109 (1976) 244.

[42] E. Witten, "Non-Abelian Bosonization in Two Dimensions," Comm. Math. Phys. 92 (1984) 455.

[43] M. B. Halpern and N. A. Obers, "New semiclassical nonabelian vertex operators for chiral and nonchiral WZW theory," Int. J. Mod. Phys. A12 (1997) 4317, hep-th/9610081.

[44] J. L. Cardy, "Conformal Invariance and Surface Critical Behavior," Nucl. Phys. B240 (1984) 514; "Boundary Conditions, Fusion Rules and the Verlinde Formula," Nucl. Phys. B324 (1989) 581.

[45] J. L. Cardy and D. C. Lewellen, "Bulk and Boundary Operators in Conformal Field Theory," Phys. Lett. B259 (1991) 274.

[46] N. Ishibashi, "The boundary and crosscap states in conformal field theories," Mod. Phys. Lett. A4 (1989) 251.

[47] J. Fuchs and C. Schweigert, "Symmetry breaking boundaries I. General theory," Nucl. Phys. B558 (1999) 419, hep-th/9902132 